\title{
Estimating the rate of plasmid transfer with an adapted Luria-Delbrück fluctuation analysis and a case study on the evolution of plasmid transfer rate
}

Olivia Kosterlitz ${ }^{1,2^{*}}$, Clint Elg²,3, Adamaris Muñiz Tirado ${ }^{1}$, Claire Wate ${ }^{1}$, Ivana Bozic ${ }^{4}$, Eva M. Top ${ }^{2,3}$, Benjamin Kerr ${ }^{1,2^{*}}$

${ }^{1}$ Biology Department, University of Washington, Seattle, WA, USA.

${ }^{2}$ BEACON Center for the Study of Evolution in Action, East Lansing, MI, USA.

${ }^{3}$ Department of Biological Sciences and Institute for Bioinformatics and Evolutionary Studies, University of Idaho, Moscow, ID, USA.

${ }^{4}$ Department of Applied Mathematics, University of Washington, Seattle, WA, USA.

*e-mail: livkost@uw.edu; kerrb@uw.edu

\section{Author contributions}

O.K. and B.K. conceived of the presented ideas. B.K. and I.B. developed the theory. O.K. developed and performed the simulations. C.E. facilitated part of the simulations. O.K. developed the experimental protocol. O.K., A.M.T., and C.W. performed the experiments. O.K. and B.K. wrote the manuscript. E.M.T. and B.K. supervised the project. All authors discussed the results and contributed to the final manuscript. 
Abstract

To increase our basic understanding of the ecology and evolution of conjugative plasmids, we need a reliable estimate of their rate of transfer between bacterial cells.

\section{Introduction}

A fundamental rule of life and the basis of heredity involves the passage of genes from parents to their offspring. Plasmids in bacteria can violate this rule of strict vertical inheritance by shuttling DNA among neighboring, non-related bacteria through horizontal gene transfer (HGT). Plasmids are independently replicating extrachromosomal DNA molecules, and some encode for their own horizontal transfer, a process termed conjugation. Bacteria containing such conjugative plasmids are governed by two modes of inheritance: vertical and horizontal. In an abstract sense, conjugation in prokaryotes is 
similar to the genetic recombination that occurs in sexually reproducing eukaryotes; specifically, conjugation is a fundamental mechanism for producing new combinations of genetic elements. Since conjugation facilitates gene flow across vast phylogenetic distances, the expansive gene repertoire in the "accessory" genome is shared among many microbial species ${ }^{1}$. While this complicates phylogenetic inference, it reinforces the notion that conjugation has a central role in shaping the ecology and evolution of microbial communities $^{2,3}$. Notably, conjugative plasmids are commonly the vehicle for the spread of antimicrobial resistance (AMR) genes and the emergence of multi-drug resistance (MDR) in clinical pathogens ${ }^{4}$. While such plasmids may impose growth costs on their bacterial hosts in the absence of antibiotics ${ }^{5}$, theoretical and experimental studies have demonstrated how conjugation itself may be a critical process for the invasion and maintenance of costly plasmids ${ }^{6,7,8}$. To model the spread of antibiotic resistance, improve phylogenetic inference, and increase our understanding of the fundamental process of conjugation and its impacts on the basic biology of bacteria, accurate measures of conjugation are of the utmost importance.

The basic approach to measure conjugation involves mixing plasmid-containing bacteria, called "donors", with plasmid-free bacteria, called "recipients". After donors and recipients are incubated together for some period of time, which is hereafter denoted as $\tilde{t}$, the population is assessed for recipients that have received the plasmid from the donor; these transformed recipients are called "transconjugants". To describe conjugation the earliest and some of the most widely used approaches measure the densities of donors, recipients, and transconjugants after the incubation time $\tilde{t}$ ( $D_{\tilde{t}}, R_{\tilde{t}}$, and $T_{\tilde{t}}$, respectively) to calculate various ratios (i.e., conjugation frequency or efficiency) including $T_{\tilde{t}} / R_{\tilde{t}}, T_{\tilde{t}} / D_{\tilde{t}}$, $T_{\tilde{t}} /\left(D_{\tilde{t}}+R_{\tilde{t}}+T_{\tilde{t}}\right)^{9}$, see Zhong et. al. ${ }^{10}$ and Huisman et. al. ${ }^{11}$ for a comprehensive overview. These conjugation ratios are descriptive statistics and have been shown to vary with initial densities $\left(D_{0}\right.$ and $\left.R_{0}\right)$ and incubation time $(\tilde{t})^{10,11}$. While these statistics may be useful for within study comparisons of the combined processes of conjugation and transconjugant growth, they are not estimating the rate of conjugation events. To see this, consider two plasmids, $\mathrm{A}$ and $\mathrm{B}$, and assume both have the same conjugation rate, but plasmid $A$ results in a higher transconjugant growth rate. Assuming all other parameters (e.g., donor growth, recipient growth, etc.) are the same and growth occurs during the assay, all of the above ratios would be higher for $A$, even though its conjugation rate 
doesn't differ from plasmid B. Clearly, an estimate of the rate of conjugation itself is desirable.

The conjugation rate (i.e., plasmid transfer rate) is the "gold-standard" measurement to describe conjugation akin to measuring other fundamental rate parameters, such as growth rate or mutation rate. Such metrics are useful for crossliterature comparison to understand basic microbial ecology and evolution ${ }^{12}$. There are only a few widely used methods to measure conjugation rate, which all are derived from the Levin et. al. ${ }^{13}$ mathematical model describing the change in density of donors, recipients and transconjugants over time (given by dynamic variables $D_{t}, R_{t}$, and $T_{t}$, respectively). In this model, each population type grows exponentially at the same growth rate $\psi$. In addition, the transconjugant density increases as a result of conjugation events both from donors to recipients and from existing transconjugants to recipients at the same conjugation rate $\gamma$. The recipient density decreases due to these conjugation events. The densities of these dynamic populations are described by the following differential equations (where the $t$ subscript is dropped from the dynamic variables for notational convenience):

$$
\begin{gathered}
\frac{d D}{d t}=\psi D, \\
\frac{d R}{d t}=\psi R-\gamma R(D+T), \\
\frac{d T}{d t}=\psi T+\gamma R(D+T) .
\end{gathered}
$$

Each current rate estimation method solved the set of ordinary differential equations from the Levin et. al. model (or a slight variation) to find an estimate for the conjugation rate $\gamma$. These estimates differ by the assumptions used to find the analytical solution. Two estimates for conjugation rate standout as widely used in the literature ${ }^{7,14,15}$. Levin et. al. ${ }^{13}$ derived the first of these estimates for conjugation rate $\gamma$ in terms of the density of donors, recipients, and transconjugants $\left(D_{\tilde{t}}, R_{\tilde{t}}\right.$, and $T_{\tilde{t}}$, respectively) after the incubation time $\tilde{t}$ :

$$
\gamma=\frac{T_{\tilde{t}}}{D_{\tilde{t}} R_{\tilde{t}} \tilde{t}} .
$$

We label the expression in equation [4] as the "TDR" estimate for the conjugation rate, where TDR stands for the dynamic variables used in the estimate. Besides the model's homogeneous growth rates and conjugation rates, the most notable assumption used in 
91 the TDR derivation is that there is little to no change in the population densities due to growth. Thus, laboratory implementation that respects this assumption can be difficult; albeit chemostats and other implementation methods have been developed to circumvent this constraint 7,13 .

Simonsen et. al. ${ }^{16}$ derived the second of these widely used estimates for conjugation rate $\gamma$, which importantly expands application beyond the TDR method by allowing for population growth. In addition to $D_{\tilde{t}}, R_{\tilde{t}}$, and $T_{\tilde{t}}$, this estimate depends on the initial and final density of all bacteria $\left(N_{0}\right.$ and $N_{\tilde{t}}$, respectively) as well as the population growth rate $(\psi)$.

$$
\gamma=\psi \ln \left(1+\frac{T_{\tilde{t}}}{R_{\tilde{t}}} \frac{N_{\tilde{t}}}{D_{\tilde{t}}}\right) \frac{1}{\left(N_{\tilde{t}}-N_{0}\right)}
$$

100

101

102

103

We term equation [5] as the "SIM" estimate for the conjugation rate, where SIM stands for "Simonsen et. al. Identicality Method" since the underlying model still assumes all strains are identical with regards to growth rates and conjugation rates. The laboratory implementation for the SIM is straightforward, requiring measurements of cell densities over some period of time. Because several of these densities are acquired at the end of an assay, the SIM is often referred to as "the end-point method". However, we forego this term since other methods we discuss are also end-point methods.

Huisman et. al. ${ }^{11}$ recently updated the SIM estimate, further extending its application by relaxing the assumption of identical growth and transfer rates for all strains. Specifically, their model extension allowed each strain to have its own growth rate $\left(\psi_{D}\right.$, $\psi_{R}$, and $\psi_{T}$ denote the growth rates for donors, recipients, and transconjugants, respectively) and each plasmid-bearing strain to have a distinct transfer rate ( $\gamma_{D}$ and $\gamma_{T}$ denote the rate of conjugation to recipients from donors and transconjugants, respectively). They derived an estimate of $\gamma_{D}$ by assuming that the rate of production of transconjugants through cell division and conjugation from donors to recipients was much greater than conjugation from transconjugants to recipients (e.g., $\gamma_{T} \leq \gamma_{D}$ is sufficient to ensure this assumption under many standard assay conditions):

$$
\gamma_{D}=\left(\psi_{D}+\psi_{R}-\psi_{T}\right) \frac{T_{\tilde{t}}}{\left(D_{\tilde{t}} R_{\tilde{t}}-D_{0} R_{0} e^{\psi_{T} \tilde{t}}\right)} .
$$

We term equation [6] as the ASM estimate for donor-recipient conjugation rate, where ASM stands for "Approximate Simonsen et. al. Method". The descriptions provided for the aforementioned conjugation rate methods are cursory but see the Supplemental 
120 Information (SI section 1-6) for a more detailed historical overview, mathematical 121 derivations, laboratory implementations, and a fuller discussion of how methods compare.

122 Deterministic numerical simulations have been used to test the accuracy of the 123 aforementioned methods (TDR, SIM, and ASM), revealing important limitations of each approach ${ }^{11,16,17}$. For instance, simulations have demonstrated that all the estimates can become inaccurate when the donor-recipient conjugation rate $\left(\gamma_{D}\right.$, hereafter "donor conjugation rate" for simplicity) differs from the transconjugant-recipient conjugation rate $\left(\gamma_{T}\right.$, hereafter "transconjugant conjugation rate" for simplicity). Such a situation is likely when the donor and recipient populations are different strains or species. While deterministic simulations have revealed estimate limitations, the deterministic framework itself encompasses an additional limitation. Conjugation is an inherently stochastic process (i.e., random process), and the effects of such stochasticity on the accuracy and precision of rate estimates are currently unknown.

Here we derive a novel estimate for conjugation rate, inspired by the LuriaDelbrück fluctuation experiment, by explicitly tracking transconjugant dynamics as a stochastic process (i.e., a continuous time branching process). In addition, our method allows for unrestricted heterogeneity in growth rates and conjugation rates. Thus, our method fills a gap in the methodological toolkit by allowing accurate estimation of conjugation rates in a wide variety of strains and species. For example, over the past few years several studies have called for and recognized the importance of evaluating the biology of plasmids in microbial communities $4,18,19$. Our method is available to support this direction of research by clearly disentangling cross-species and within-species conjugation rates. Thus, our approach can provide accurate conjugation rate parameters for mechanistic models describing plasmid dynamics in microbial communities, where key assumptions of previous estimates can be violated. We used stochastic simulations to validate our estimate and compare its accuracy and precision to the aforementioned estimates. We also developed a high throughput protocol for the laboratory by using microtiter plates to rapidly screen many mating cultures (i.e., donor-recipient co-cultures) for the existence of transconjugants. Our protocol is unique compared to the other estimates and circumvents problems involving spurious laboratory results that can bias other approaches. Finally, we implement our approach to investigate the evolution of conjugation rate in an Escherichia coli host containing an IncP-1 $\beta$ conjugative plasmid. 


\section{Theoretical Framework}

Conjugation is a stochastic process that transforms the genetic state of a cell. This is analogous to mutation, which is also a process that transforms the genetic state of a cell. While mutation transforms a wild-type cell to a mutant, conjugation transforms a recipient cell to a transconjugant.

This analogy inspired us to revisit the way Luria and Delbrück handled the mutational process in their classic paper on the nature of bacterial mutation ${ }^{20}$, outlined in Figure 1a-d. For this process, assume that the number of wild-type cells, $N_{t}$, is expanding exponentially. Let the rate of mutant formation be given by $\mu$. In Figure 1a, we see that the number of mutants in a growing population increases due to mutation events (highlighted purple cells) and due to faithful reproduction by mutants (non-highlighted purple cells). The rate at which mutants are generated (highlighted purple cells) is $\mu N_{t}$, which grows as the number of wild-type cells increases (Figure 1b). However, the rate of transformation per wild-type cell is the mutation rate $\mu$, which is constant (Figure 1c). Since mutations are random, parallel cultures will vary in the number of mutants depending if and when mutation events occur. As seen in Figure 1d, for sufficiently small wild-type populations growing over sufficiently small periods, some replicate populations will not contain any mutant cell (gray shading) while other populations exhibit mutants (yellow shading). Indeed, the cross-replicate fluctuation in the number of mutants was a critical component of the Luria-Delbrück experiment.

To apply this strategy to estimate the conjugation rate, we can similarly think about an exponentially growing population of recipients (Figure 1e). But now there is another important cell population present (the donors). The transformation of a recipient is simply the generation of a transconjugant (highlighted purple cells) via conjugation with a donor. If we ignore conjugation from transconjugants, the rate at which transconjugants are generated is $\gamma_{D} D_{t} R_{t}$ (Figure 1f). However, when we consider the rate of transformation per recipient cell, we do not have a constant rate. Rather, this transformation rate per recipient is $\gamma_{D} D_{t}$, which grows with the donor population (Figure 1g). It is as if we are tracking a mutation process where the mutation rate is exponentially increasing. Yet the rate of transformation per recipient and donor is constant, which is the donor conjugation rate $\gamma_{D}$. As with mutation, conjugation is random which results in a distribution in the 
bioRxiv preprint doi: https://doi.org/10.1101/2021.01.06.425583; this version posted December 3, 2021. The copyright holder for this preprint (which was not certified by peer review) is the author/funder, who has granted bioRxiv a license to display the preprint in perpetuity. It is made available under aCC-BY-NC-ND 4.0 International license.

number of transconjugants among parallel cultures depending on the time points at which

will exhibit transconjugants (yellow shading).

a

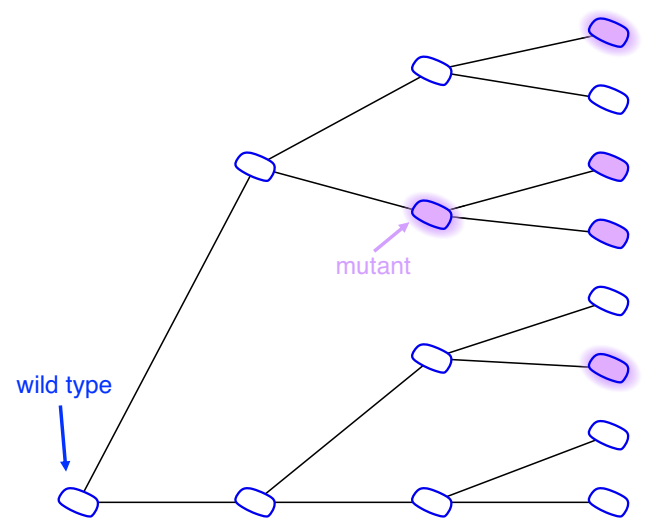

b

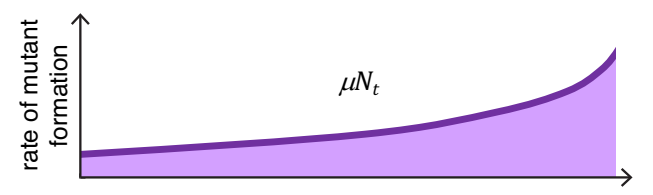

C

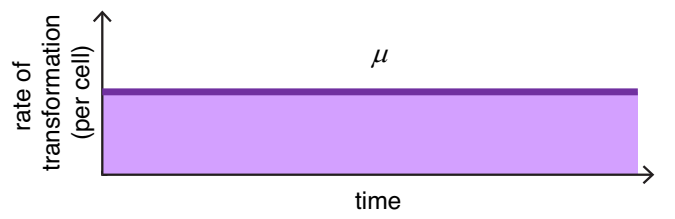

d
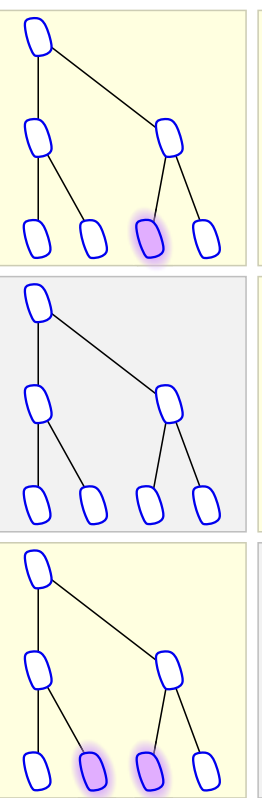

Mutation

time
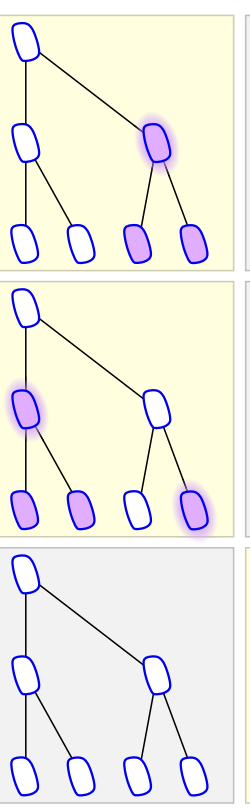

e

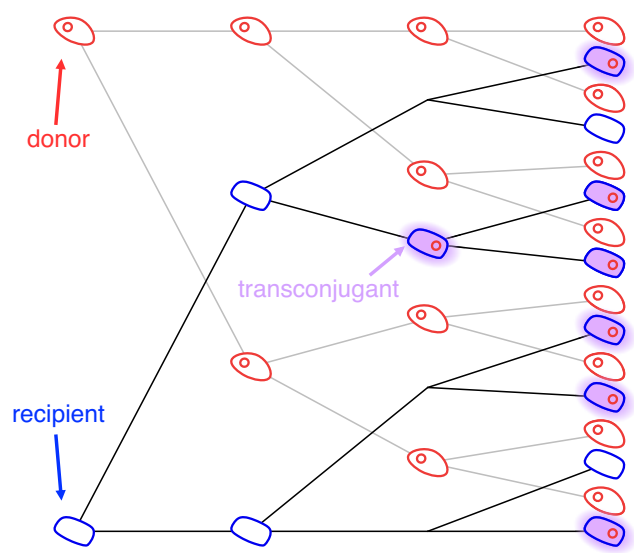

f

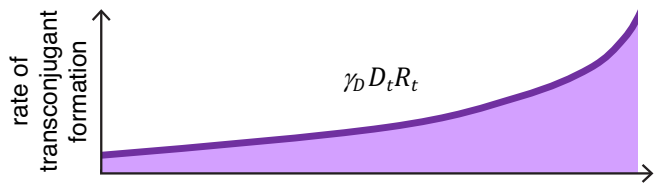

g

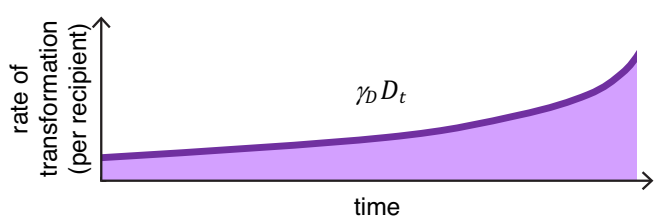

h
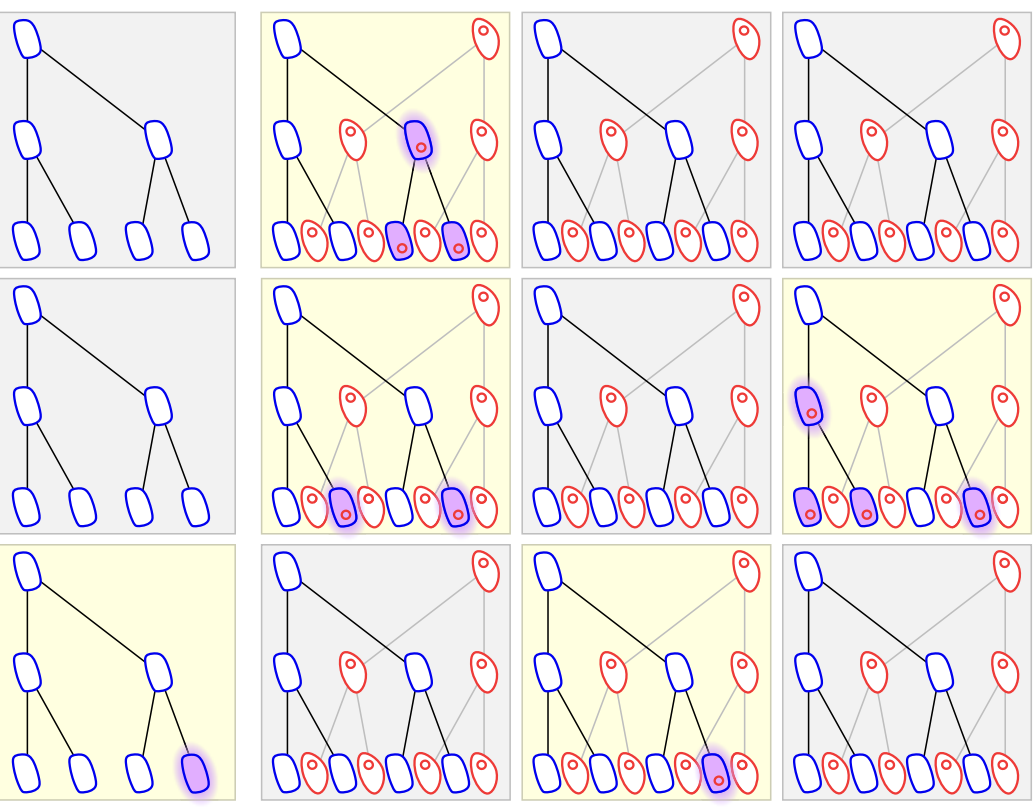

Figure 1: Schematic comparing the process of mutation (a-d) to the process of conjugation (e-h). (a) In a growing population of wild-type cells, mutants arise (highlighted purple cells) and reproduce (non-highlighted purple cells). (b) The rate at which mutants are generated grows as the number of wild-type cells increases at rate $\mu N_{t}$. (c) The rate of transformation per wild-type cell is the mutation rate $\mu$. (d) Wild-type cells growing in 9 separate populations where mutants arise in a proportion of the populations (yellow background) at different cell divisions. Here, for each tree, time 
progresses from top to bottom (rather than left to right as in part a). (e) In a growing population of donors and recipients, transconjugants arise (highlighted purple cells) and reproduce (non-highlighted purple cells). (f) The rate at which transconjugants are generated grows as the numbers of donors and recipients increase at rate $\gamma_{D} D_{t} R_{t}$. $(\mathrm{g})$ The rate of transformation per recipient cell grows as the number of donors increases at a rate $\gamma_{D} D_{t}$, where $\gamma_{D}$ is the constant conjugation rate. (h) Donor and recipient cells growing in 9 separate populations where transconjugants arise in a proportion of the populations (yellow background) at different points in time (which again, here, progresses top to bottom for each tree).

Using this analogy, here we describe a new approach for estimating conjugation rate which embraces conjugation as a stochastic process ${ }^{21}$. Let the density of donors, recipients and transconjugants in a well-mixed culture at time $t$ be given by the variables $D_{t}, R_{t}$, and $T_{t}$. In all that follows, we will assume that the culture is inoculated with donors and recipients, while transconjugants are initially absent (i.e., $D_{0}>0, R_{0}>0$, and $T_{0}=$ $0)$. The donor and recipient populations grow according to the following standard exponential growth equations

$$
\begin{aligned}
& D_{t}=D_{0} e^{\psi_{D} t} \\
& R_{t}=R_{0} e^{\psi_{R} t}
\end{aligned}
$$

where $\psi_{D}$ and $\psi_{R}$ are the growth rates for donor and recipient cells, respectively. With equations [7] and [8], we are making a few assumptions, which also occur in some of the previous methods (see SI Table 3 for a comparison). First, we assume that donors and recipients grow exponentially. Given that we focus on low-density culture conditions where nutrients are plentiful, this assumption will be reasonable. Second, we assume that population growth for these two strains is deterministic (i.e., $D_{t}$ and $R_{t}$ are not random variables). As long as the numbers of donors and recipients are not too small (i.e., $D_{0} \gg$ 0 and $R_{0} \gg 0$ ), this assumption is acceptable. Lastly, we assume the loss of recipient cells to transformation into transconjugants can be ignored. For what follows, the rate of generation of transconjugants per recipient cell (as in Figure 1g) is very small relative to the per capita recipient growth rate, making this last assumption justifiable.

The population growth of transconjugants is modeled using a continuous-time stochastic process. The number of transconjugants, $T_{t}$, is a random variable taking on non-negative integer values. In this section, we will assume the culture volume is $1 \mathrm{ml}$ and thus the number of transconjugants (i.e., cfu) is equivalent to the density of transconjugants (i.e., cfu per $\mathrm{ml}$ ). For a very small interval of time, $\Delta t$, the current number of transconjugants will either increase by one or remain constant. The probabilities of each possibility are given as follows: 


$$
\begin{gathered}
\operatorname{Pr}\left\{T_{t+\Delta t}=T_{t}+1\right\}=\gamma_{D} D_{t} R_{t} \Delta t+\gamma_{T} T_{t} R_{t} \Delta t+\psi_{T} T_{t} \Delta t \\
\operatorname{Pr}\left\{T_{t+\Delta t}=T_{t}\right\}=1-\left(\gamma_{D} D_{t} R_{t}+\gamma_{T} T_{t} R_{t}+\psi_{T} T_{t}\right) \Delta t .
\end{gathered}
$$

The three terms on the right-hand side of equation [9] illustrate the processes enabling the transconjugant population to increase. The first term gives the probability that a donor transforms a recipient into a transconjugant via conjugation $\left(\gamma_{D}\right)$. The second term gives the probability that a transconjugant transforms a recipient via conjugation $\left(\gamma_{T}\right)$. The third term measures the probability that a transconjugant cell divides $\left(\psi_{T}\right.$ is the transconjugant growth rate). Equation [10] is simply the probability that none of these three processes occur.

We focus on a situation where there are no transconjugants. Therefore, the only process that can change the number of transconjugants is conjugation of the plasmid from a donor to a recipient. Using equation [10] with $T_{t}=0$, we have

$$
\operatorname{Pr}\left\{T_{t+\Delta t}=0 \mid T_{t}=0\right\}=1-\gamma_{D} D_{t} R_{t} \Delta t
$$

We let the probability that we have zero transconjugants at time $t$ be denoted by $p_{0}(t)$ (i.e., $p_{0}(t)=\operatorname{Pr}\left\{T_{t}=0\right\}$ ). In SI section 7, we derive the following expression for $p_{0}(t)$ at time $t=\tilde{t}$ :

$$
p_{0}(\tilde{t})=\exp \left\{\frac{-\gamma_{D} D_{0} R_{0}}{\psi_{D}+\psi_{R}}\left(e^{\left(\psi_{D}+\psi_{R}\right) \tilde{t}}-1\right)\right\} .
$$

This expression is similar in form to the mutation rate derived by Luria and Delbrück in their classic paper on the nature of bacterial mutation, which is not a coincidence.

In SI section 8, we rederive the Luria-Delbrück result, which can be expressed as

$$
\mu=-\ln p_{0}(\tilde{t})\left(\frac{\psi_{N}}{N_{0}\left(e^{\psi_{N} \tilde{t}}-1\right)}\right) .
$$

In the mutational process modeled by Luria and Delbrück, $N_{0}$ is the initial wild-type population size, which grows exponentially at rate $\psi_{N}$. For Luria and Delbrück, $p_{0}(\tilde{t})$ refers to the probability of zero mutants at time $\tilde{t}$ (as in a gray-shaded tree in Figure 1d), whereas $p_{0}(\tilde{t})$ in the conjugation estimate refers to the probability of zero transconjugants (as in a gray-shaded tree in Figure 1h). Comparing equation [14] to equation [13], conjugation 
235 can be thought of as a mutation process with initial wild-type population size $D_{0} R_{0}$ that grows at rate $\psi_{D}+\psi_{R}$. We explore further the connection between mutation and conjugation in SI section 13.

We label the expression in equation [13] as the LDM estimate for donor conjugation rate, where LDM stands for "Low Density Method" as the assumption of exponential growth is well grounded when the cell populations are growing at low density. Indeed, this is how we conduct the assay to compute this estimate. The acronym can alternatively stand for "Luria-Delbrück Method" given the connection to their approach.

\section{Simulation Methods}

To explore the accuracy and precision of the LDM estimate (as well as other estimates), we developed a stochastic simulation framework using the Gillespie algorithm. We extended the base model (equations [1] - [3]) to include plasmid loss due to segregation at rate $\tau$. Specifically, transconjugants are transformed into plasmid-free recipients due to plasmid loss via segregation at rate $\tau_{T}$. Similarly, the donors become plasmid-free cells through segregative loss of the plasmid at rate $\tau_{D}$; therefore, the extended model tracks the change in density of a new population type, plasmid-free former donors $(F)$. In total, the extended model describes the density changes in four populations $(D, R, T$, and $F)$ influenced by the values of various biological parameters: growth rates $\left(\psi_{D}, \psi_{R}, \psi_{T}\right.$, and $\left.\psi_{F}\right)$, conjugation rates $\left(\gamma_{D}\right.$ and $\left.\gamma_{T}\right)$ and segregation rates $\left(\tau_{D}\right.$ and $\left.\tau_{T}\right)$. For each simulation, we specify starting cell densities $\left(D_{0}>0\right.$ and $R_{0}>0$, but always $T_{0}=F_{0}=0$ ) and allow the four populations to grow and transform stochastically for a specified incubation time $\tilde{t}$ in a $1 \mathrm{ml}$ culture volume. The simulations occur in sets, where each set involves a sweep across values of some key parameter. Each sweep varies a biological process (i.e., growth, conjugation, or segregation) in isolation to determine the effect on accuracy and precision of the donor conjugation rate estimates. For instance, the transconjugant conjugation rate $\left(\gamma_{T}\right)$ could be varied while all other parameters are held constant. For each parameter setting within a sweep, ten thousand parallel populations were simulated, and then conjugation rate was calculated to yield replicate estimates for each method of interest (TDR, SIM, ASM, and LDM). Notably, the LDM requires an estimate of the probability that a population has no transconjugants at the end of the assay, which we denote $\hat{p}_{0}(\tilde{t})$. The maximum likelihood 
267 estimate of this probability is the fraction of populations (i.e., parallel simulations) that

268 have no transconjugants at the specified incubation time $\tilde{t}$ (see SI section 9 for details). Thus, for LDM, we calculated $\hat{p}_{0}(\tilde{t})$ at the specified incubation time $\tilde{t}$ by reserving multiple parallel populations for each conjugation rate estimate. For more details on the simulations such as the extended model equations, selection of the specified incubation time $\tilde{t}$, and calculation of each estimate see SI section 11 .

\section{Simulation Results}

Exploring accuracy and sensitivity of the LDM and other conjugation methods

The underlying assumptions used to derive the various estimates for conjugation rate (i.e., TDR, SIM, ASM, and LDM) entail simplifications of biological systems. To explore the effects of violating these assumptions on the LDM and other conjugation rate estimates, we used a stochastic simulation framework to investigate their accuracy across a range of theoretical conditions. All of our simulated populations increase in size over the incubation time, so a fundamental assumption of the TDR approach (i.e., no change in the density due to growth) is broken for all of the runs. Here we divide our simulations into three scenarios that break additional assumptions of at least one conjugation model considered to this point: unequal growth rates, unequal conjugation rates, and a non-zero segregation rate. When growth rates are different between populations, a modeling assumption is violated for the TDR and SIM estimates. When donor and transconjugant conjugation rates are unequal, a modeling assumption can be violated for the TDR, SIM, and ASM estimates. When the segregation rate is non-zero, a modeling assumption is violated for all of the estimates considered. We note that some of our parameter values fall outside of reported values; these choices were made either to improve computational speed or to better illustrate the effects of differences (e.g., in growth or transfer rates). However, many of the parameter values used to explore the effects of incubation time were set to reported parameter values.

\section{The effect of unequal growth rates}

Plasmid-containing hosts often incur a cost or gain a benefit in terms of growth rate for plasmid carriage relative to plasmid-free hosts. We simulated a range of growth-rate 
299 effects on plasmid-containing hosts from large plasmid costs $\left(\psi_{D}=\psi_{T} \ll \psi_{R}\right)$ to large 300 plasmid benefits $\left(\psi_{D}=\psi_{T} \gg \psi_{R}\right)$. The LDM estimate had high accuracy and precision 301 across all parameter settings (Figure 2a). The ASM estimate performed similarly to the 302 LDM estimate with regards to the mean value for each parameter setting (Figure 2a, black horizontal lines). Given that the ASM was developed to allow for unequal growth rates, its mean accuracy conforms to expectations. However, it is also worth noting that the distribution of the ASM estimate was skewed for some of the parameter settings. In other words, the mean (Figure 2a, black horizontal lines) is separated from the median (Figure $2 \mathrm{a}$, green horizontal line); therefore, the average of a small number of replicates could have led to a deviant mean estimate. The TDR and SIM estimates were inaccurate for some to all of the parameter settings and also had skewed distributions for some parameter settings. With regards to precision, the LDM estimate outperformed the other estimates due to a smaller variance at each parameter setting.

The effect of unequal conjugation rates

The conjugation rate can vary between different bacterial strains and species. We scenarios where growth rates can differ between donors, recipients and transconjugants. In fact, often it is the case that donors and recipients are different strains or species. We simulated donors growing faster $\left(\psi_{D}>\psi_{T}=\psi_{R}\right)$ or slower $\left(\psi_{D}<\psi_{T}=\psi_{R}\right)$ than recipients and transconjugants. Similar to the plasmid carriage scenario, the LDM exhibited high accuracy and precision relative to other metrics (SI Figure 1). explored different parameter settings again, ranging from a relatively low transconjugant conjugation rate $\left(\gamma_{T} \ll \gamma_{D}\right)$ to a relatively high transconjugant conjugation rate $\left(\gamma_{T} \gg \gamma_{D}\right)$ compared to the donor conjugation rate. The LDM estimate had high accuracy and precision across all parameter settings and outperformed the other estimates, especially when transconjugant conjugation rate greatly exceeded donor conjugation rate (Figure $2 b)$. This result aligns with our expectation given that a relatively high transfer rate from transconjugants to recipients can violate a modeling assumption for all the other estimates. 
bioRxiv preprint doi: https://doi.org/10.1101/2021.01.06.425583; this version posted December 3, 2021. The copyright holder for this preprint (which was not certified by peer review) is the author/funder, who has granted bioRxiv a license to display the preprint in perpetuity. It is made available under aCC-BY-NC-ND 4.0 International license.

a

Unequal growth rates $\left(\psi_{D}=\psi_{T} \neq \psi_{R}\right)$

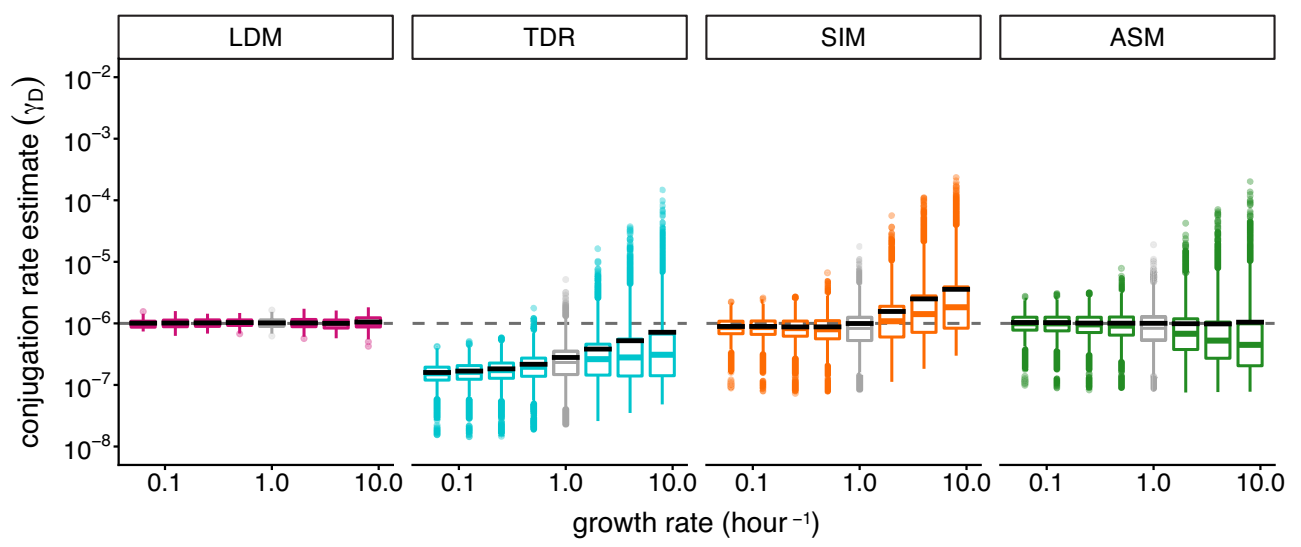

b

Unequal conjugation rates $\left(\gamma_{D} \neq \gamma_{T}\right)$

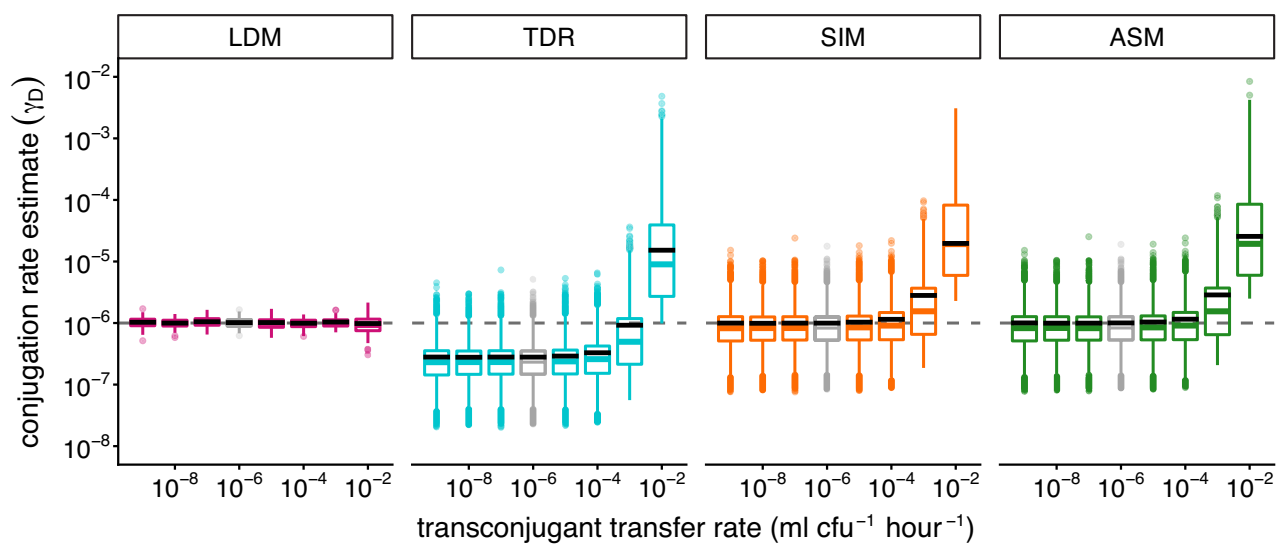

C

Non-zero segregation rates $\left(\tau_{D}=\tau_{T}>0\right)$

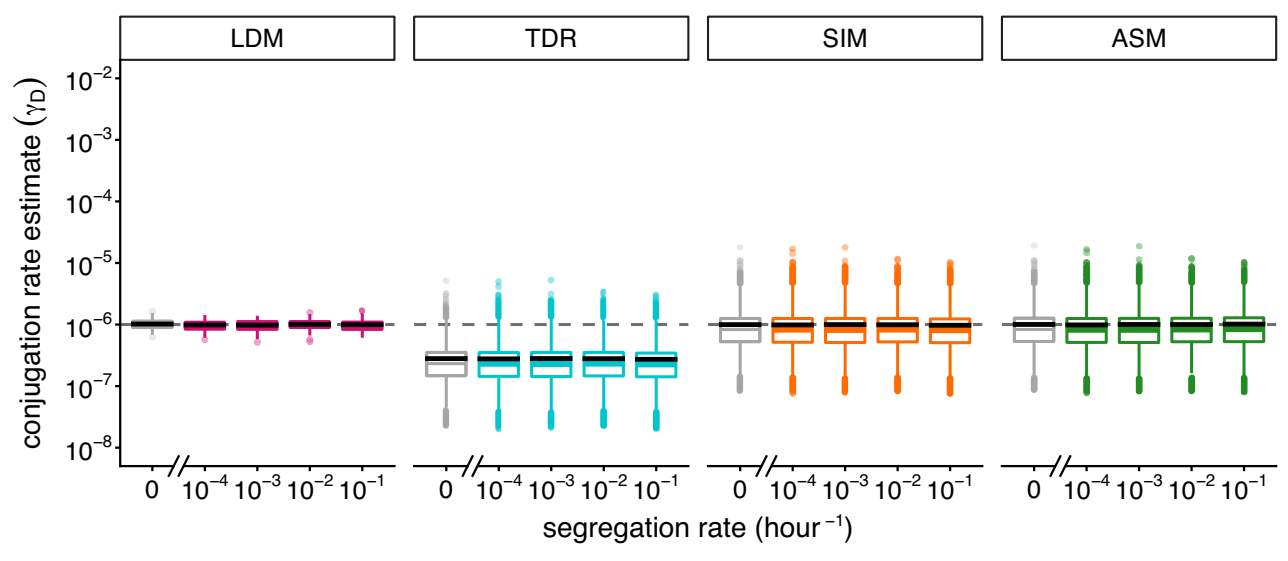

Figure 2 : The effect of violating various modeling assumptions on estimating conjugation rate. The Gillespie algorithm was used to simulate population dynamics. The parameter values were $\psi_{D}=\psi_{R}=\psi_{T}=1, \gamma_{D}=\gamma_{T}=$ $1 \times 10^{-6}$, and $\tau_{D}=\tau_{T}=0$ unless otherwise indicated. The dynamic variables were initialized with $D_{0}=R_{0}=1 \times 10^{2}$ and $T_{0}=F_{0}=0$. All incubation times are short but are specific to each parameter setting (see SI section 11 for details). Conjugation rate was estimated using 10,000 populations and summarized using boxplots. The gray dashed line indicates the "true" value for the donor conjugation rate. The box contains the $25^{\text {th }}$ to $75^{\text {th }}$ percentile. The vertical lines connected to the box contain 1.5 times the interquartile range above the $75^{\text {th }}$ percentile and below the $25^{\text {th }}$ percentile with the caveat that the whiskers were always constrained to the range of the data. The colored line in the box indicates the median. The solid black line indicates the mean. The boxplots in gray indicate the baseline parameter setting, and all colored plots represent deviation of one or two parameters from baseline. (a) Unequal growth rates were explored over a range of growth rates for the plasmid-bearing strains, namely $\psi_{D}=\psi_{T} \in\{0.0625,0.125,0.25,0.5,1,2,4,8\}$. (b) Unequal conjugation rates were probed over a range of transconjugant conjugation rates, namely $\gamma_{T} \in$ $\left\{10^{-9}, 10^{-8}, 10^{-7}, 10^{-6}, 10^{-5}, 10^{-4}, 10^{-3}, 10^{-2}\right\}$. (c) We also explored the process of segregation by considering a range of segregative loss rates $\tau_{D}=\tau_{T} \in\{0,0.0001,0.001,0.001,0.01\}$. 
The effect of a non-zero segregation rate

Plasmid loss due to segregation is a common occurrence in plasmid populations and violates a model assumption underlying all the conjugation rate estimates. We simulated a range of segregative loss rates, ranging from low $\left(\tau_{D}=\tau_{T}=0.0001\right)$ to high $\left(\tau_{D}=\tau_{T}=0.1\right)$. The LDM had high accuracy and precision across all parameter settings (Figure 2c). The effect of segregation was undetectable even for an extremely high segregation rate $\left(\tau_{D}=\tau_{T}=0.1\right)$. Similarly, the effect of segregative loss was undetectable on the other conjugation estimates compared to their performance without any segregative loss (Figure 2c, colored vs. grey box plots). Thus, we find that all estimates appear robust with regards to an introduction of the process of segregation.

\section{Case studies to explore the effects of incubation time}

The incubation time $\tilde{t}$ is an important consideration for executing all conjugation rate assays in the laboratory. Often the incubation time is variable for estimates of conjugation rate reported in the literature. We explored the effects of altering incubation time by estimating conjugation rate at 30-minute intervals. Since these estimates rely on measuring different quantities, the range for the 30-minute sampling intervals differ between estimates. For the TDR, SIM, and ASM estimates, the range for these intervals was determined by the condition that at least 90 percent of the simulated populations contained transconjugants at the incubation time $\tilde{t}$. Specifically, we note that equations [4], [5], and [6] all require $T_{\tilde{t}}>0$ for a non-zero conjugation rate estimate. This contrasts with the LDM since this estimate needs some parallel populations to be absent of transconjugants. Specifically, equation [13] requires $0<p_{0}(\tilde{t})<1$ for a non-zero finite conjugation rate estimate. Thus, for the LDM estimate, the range for the sampling intervals was determined by the condition that at least one parallel population had zero transconjugants. Therefore, estimates for the LDM are shown at earlier incubation times than those for the other methods (Figure 3). 
bioRxiv preprint doi: https://doi.org/10.1101/2021.01.06.425583; this version posted December 3, 2021. The copyright holder for this preprint (which was not certified by peer review) is the author/funder, who has granted bioRxiv a license to display the preprint in perpetuity. It is made available under aCC-BY-NC-ND 4.0 International license.

a

Unequal growth rate $\left(\psi_{D}=\psi_{T}<\psi_{R}\right)$

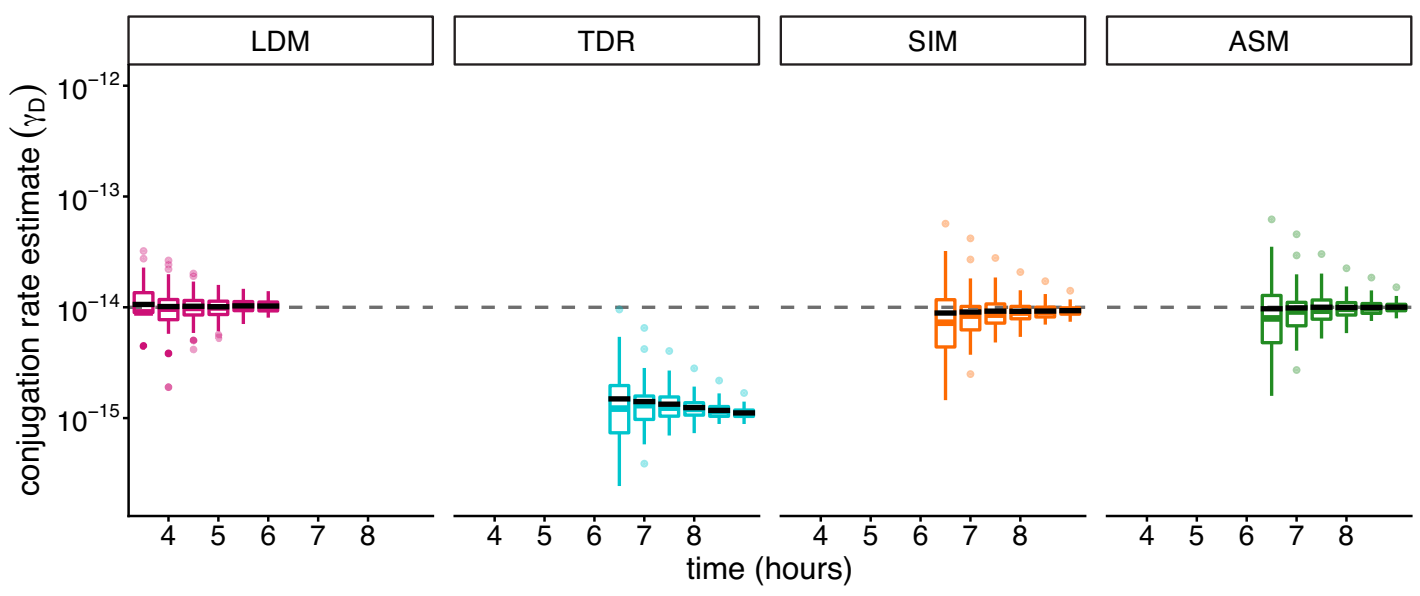

b

\section{Unequal conjugation rate $\left(\gamma_{D}<\gamma_{T}\right)$}

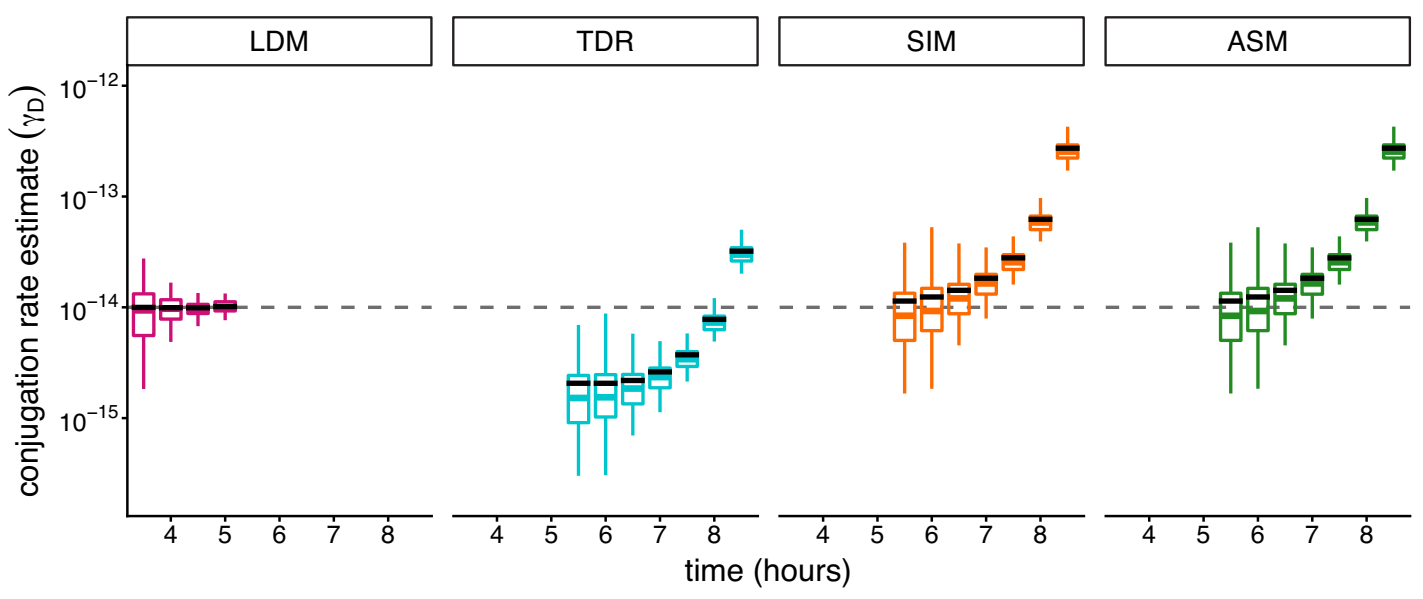

c

Non-zero segregation rates $\left(\tau_{D}=\tau_{T}>0\right)$

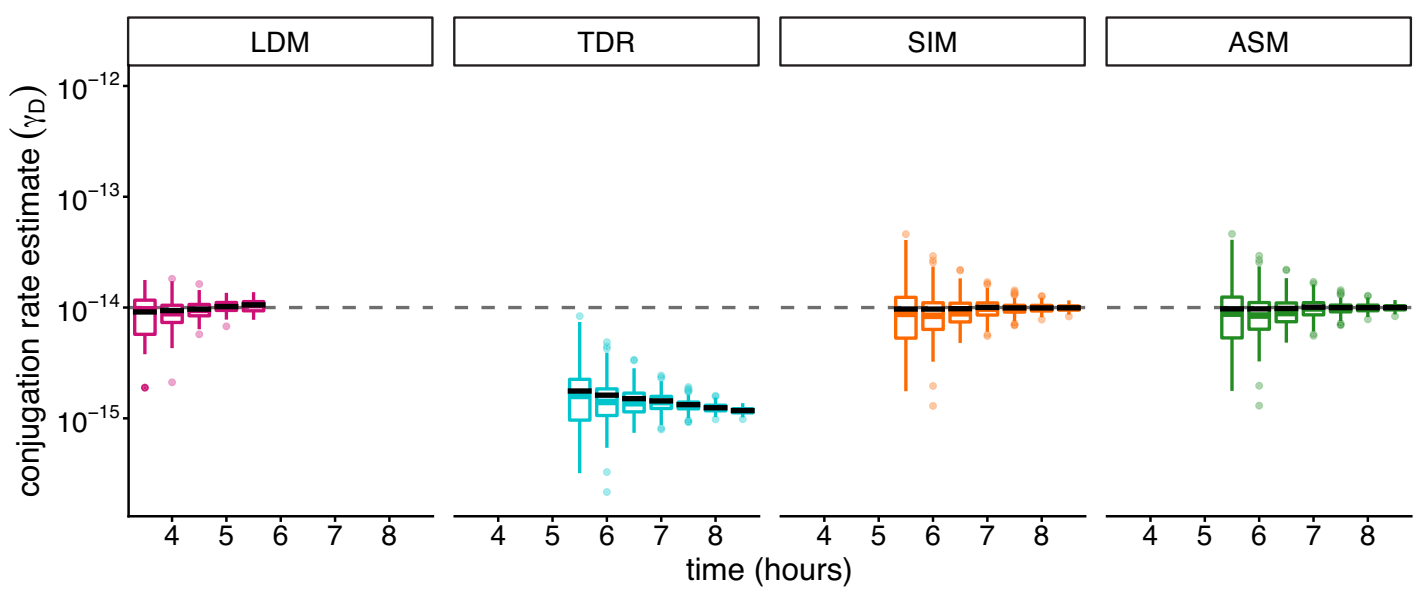

Figure 3 : The effect of incubation time $(\tilde{\boldsymbol{t}})$ on estimating conjugation rate. The Gillespie algorithm was used to simulate population dynamics with $\psi_{D}=\psi_{R}=\psi_{T}=1, \gamma_{D}=\gamma_{T}=1 \times 10^{-14}$, and $\tau_{D}=\tau_{T}=0$ unless otherwise indicated. The dynamic variables were initialized with $D_{0}=R_{0}=1 \times 10^{5}$ and $T_{0}=F_{0}=0$. Conjugation rate was estimated for 100 populations and summarized using boxplots. The gray dashed line indicates the "true" value for the donor conjugation rate. Each box contains the $25^{\text {th }}$ to $75^{\text {th }}$ percentile. The vertical lines connected to the box contain 1.5 times the interquartile range above the $75^{\text {th }}$ percentile and below the $25^{\text {th }}$ percentile with the caveat that the whiskers were always constrained to the range of the data. The colored line in the box indicates the median. The solid black line indicates the mean. (a) An unequal growth rate was simulated with $\psi_{D}=\psi_{T}=0.07$. (b) An unequal conjugation rate was simulated with $\gamma_{T}=1 \times 10^{-9}$. (c) We simulated a positive segregation rate with $\tau_{D}=\tau_{T}=0.01$. 
We ran simulations with parameter values for growth and conjugation rates which are reported in the literature $\left(\psi_{D}=\psi_{R}=\psi_{T}=\psi_{F}=1, \gamma_{D}=\gamma_{T}=1 \times 10^{-14}\right)$ and reasonable initial densities $\left(D_{0}=R_{0}=1 \times 10^{5}\right)$. As before, each biological scenario examined a perturbation to a single biological parameter (i.e., growth, conjugation, or segregation) to determine the effect on accuracy and precision. For the scenario exploring growth differences, we added a plasmid cost (Figure $3 a, \psi_{D}=\psi_{T}=0.7$ ). For the scenario highlighting unequal conjugation rates, we increased the conjugation rate of transconjugants (Figure $3 \mathrm{~b}, \gamma_{T}=1 \times 10^{-9}$ ). For the scenario incorporating segregation, we simply chose a rather high segregation rate (Figure $3 c, \tau_{D}=\tau_{T}=0.01$ ).

The LDM estimate had high accuracy over all time points for all scenarios with precision increasing through time. The other estimates also became more precise over

\section{The LDM Implementation}

In this section we describe the general experimental procedure for estimating donor conjugation rate $\left(\gamma_{D}\right)$ using the LDM estimate in the laboratory. The assay can accommodate a wide variety of microbial species and conjugative plasmids since our estimate allows for distinct growth and conjugation rates. In SI section 6, we rearrange equation [13] to this alternative form for implementing the LDM in the laboratory:

$$
\gamma_{D}=f\left\{\frac{1}{\tilde{t}}\left[-\ln \hat{p}_{0}(\tilde{t})\right] \frac{\ln D_{\tilde{t}} R_{\tilde{t}}-\ln D_{0} R_{0}}{D_{\tilde{t}} R_{\tilde{t}}-D_{0} R_{0}}\right\} .
$$

Similar to previous conjugation estimates, the LDM requires measurement of initial and final densities of donors and recipients $\left(D_{0}, R_{0}, D_{\tilde{t}}\right.$, and $\left.R_{\tilde{t}}\right)$. In addition, the LDM requires an estimate of the probability that a population has no transconjugants at the end of the assay, which we denote $\hat{p}_{0}(\tilde{t})$. The maximum likelihood estimate of this probability is the fraction of populations (i.e., parallel mating cultures) that have no transconjugants at the specified incubation time $\tilde{t}$ (see SI section 9 for details). Cell densities are generally measured in $\frac{\mathrm{cfu}}{\mathrm{ml}}$ units and the conjugation rate in $\frac{\mathrm{ml}}{\mathrm{h} \cdot \mathrm{cfu}}$ units, thus, the standard unit of volume in these assays is a milliliter. If exactly $1 \mathrm{ml}$ is used as the volume for each mating culture (as assumed in previous sections), then the quantity in braces in equation [15] is 
the estimate for the conjugation rate. However, smaller mating volumes can be advantageous (e.g., $100 \mu \mathrm{l}$ in a well inside a microtiter plate). When the mating volume deviates from $1 \mathrm{ml}$, we must add a correction factor $f$ which is the number of experimental volume units (evu) per $\mathrm{ml}$ (e.g., for a mating volume of $100 \mu \mathrm{l}$, we would have $f=10 \frac{\mathrm{evu}}{\mathrm{ml}}$, see SI section 10 for details).

For a given incubation time $(\tilde{t})$ and initial densities of donors $\left(D_{0}\right)$ and recipients $\left(R_{0}\right)$, there will be some probability that transconjugants form $\left(1-p_{0}(\tilde{t})\right)$. The combinations of times and densities where this probability is not close to zero or one is hereafter referred to as "the conjugation window". That is, for these time-density combinations, there is a good chance that some mating cultures will result in transconjugants, while others do not. For fixed initial donor and recipient densities, the conjugation window refers to the time period where transconjugants are first expected to form. Determining the conjugation window provides the researcher with target initial densities of donors $\left(D_{0}^{\prime}\right)$ and recipients $\left(R_{0}^{\prime}\right)$ and target incubation times $\left(\tilde{t}^{\prime}\right)$. Note we add primes to the target time-density combination to set them apart from $D_{0}, R_{0}$, and $\tilde{t}$ in equation [15] which will be gathered in the conjugation assay itself.

\section{7}

To determine the conjugation window and find a time-density combination, we mix exponentially growing populations of donors and recipients in a large array of parallel mating cultures for a full factorial treatment of initial densities and incubation times (Figure $4 a$ and $4 b)$. For ease of presentation, we will assume that donors and recipients start at equal proportion and we will refer to the "initial density" as the total cell density of these two strains. In our schematic, columns of a 96 deep well microtiter plate are grouped by the value of the initial density ( 3 columns per value; Figure $4 \mathrm{a}$ ) and rows are grouped by the value of the incubation time ( 2 rows per value; Figure $4 \mathrm{~b}$ ). At each time in the set of incubation times being explored, growth medium selecting for transconjugants (see SI section 12 for explanation) is added. This dilutes the mating cultures by ten-fold, which hinders further conjugation and simultaneously permits the growth of any transconjugant cells that previously formed (Figure 4b). After a longer incubation, we assess the mating cultures ( 3 columns $\times 2$ rows $=6$ wells; Figure 4c) within each time-density treatment for presence or absence of transconjugants (i.e., turbid culture or non-turbid culture). There are three possible outcomes for each treatment: all matings have transconjugants, none of the matings have transconjugants, or there is both transconjugant-containing and 
418 transconjugant-free matings. We are interested in the last outcome (Figure 4c, gray dots).

419 These treatments meet the $\hat{p}_{0}(\tilde{t})$ condition (i.e., $0<\hat{p}_{0}(\tilde{t})<1$ ) thus identifying a valid time-density combination for the LDM conjugation assay. As a general expectation, mating cultures with high donor conjugation rates $\left(\gamma_{D}\right)$ will require shorter incubation times than mating cultures with low donor conjugation rates for a given initial density. We note that there will generally be multiple time-density treatments in the conjugation window and the final treatment choice may be determined by experimental tractability. Thus, this choice is not constrained and can be made based on an incubation time that is practically feasible. In addition, the LDM does not require the initial density of donors and recipients to be identical (as was assumed for this presentation). Indeed, in certain circumstances it may be easier to have donor and recipient initial densities be unequal.

The chosen treatment yields the target time-density combination $\left(D_{0}^{\prime}, R_{0}^{\prime}, \tilde{t}^{\prime}\right)$, and we proceed with executing the LDM conjugation assay to gather all components to estimate the conjugation rate. We mix exponentially growing populations of donors and recipients, inoculate many mating cultures at the target initial density $\left(D_{0}^{\prime}+R_{0}^{\prime}\right)$ in a 96 deep well plate, and incubate in non-selective growth medium for the target incubation time $\left(\tilde{t}^{\prime}\right)$ (Figure $4 d$ ). We note that the incubation time $\tilde{t}$ may deviate slightly from the target incubation time $\tilde{t}^{\prime}$ due to timing constraints in the laboratory, but small deviations are generally permissible. Thus, the executed incubation time $\tilde{t}$ should be used to calculate the conjugation rate. To estimate the initial densities $\left(D_{0}\right.$ and $\left.R_{0}\right)$, three mating cultures at the start of the assay are diluted and plated on donor-selecting and recipient-selecting agar plates. We note that the initial densities $\left(D_{0}\right.$ and $\left.R_{0}\right)$ may deviate from the target initial densities $\left(D_{0}^{\prime}\right.$ and $\left.R_{0}^{\prime}\right)$, but small deviations are permissible. After the incubation time $\tilde{t}$, final densities $\left(D_{\tilde{t}}\right.$ and $\left.R_{\tilde{t}}\right)$ are obtained by dilution-plating from the same mating cultures. Growth medium selecting for transconjugants is subsequently added to the remaining mating cultures. To obtain $\hat{p}_{0}(\tilde{t})$, we assess the proportion of mating cultures that are non-turbid after incubation in transconjugant-selecting medium, as these cultures have no transconjugants (Figure $4 \mathrm{~d}$ ). This differs from the traditional Luria-Delbrück method since no plating is required to obtain $\hat{p}_{0}(\tilde{t})$. The binary output (turbidity vs. no turbidity) is assessed in a high throughput manner using a standard microtiter spectrophotometer. Using the obtained densities $\left(D_{0}, R_{0}, D_{\tilde{t}}\right.$, and $\left.R_{\tilde{t}}\right)$, the incubation time $(\tilde{t})$, the proportion of transconjugant-free populations $\left(\hat{p}_{0}(\tilde{t})\right)$, and correcting for the 
bioRxiv preprint doi: https://doi.org/10.1101/2021.01.06.425583; this version posted December 3, 2021. The copyright holder for this preprint (which was not certified by peer review) is the author/funder, who has granted bioRxiv a license to display the preprint in perpetuity. It is made available under aCC-BY-NC-ND 4.0 International license.

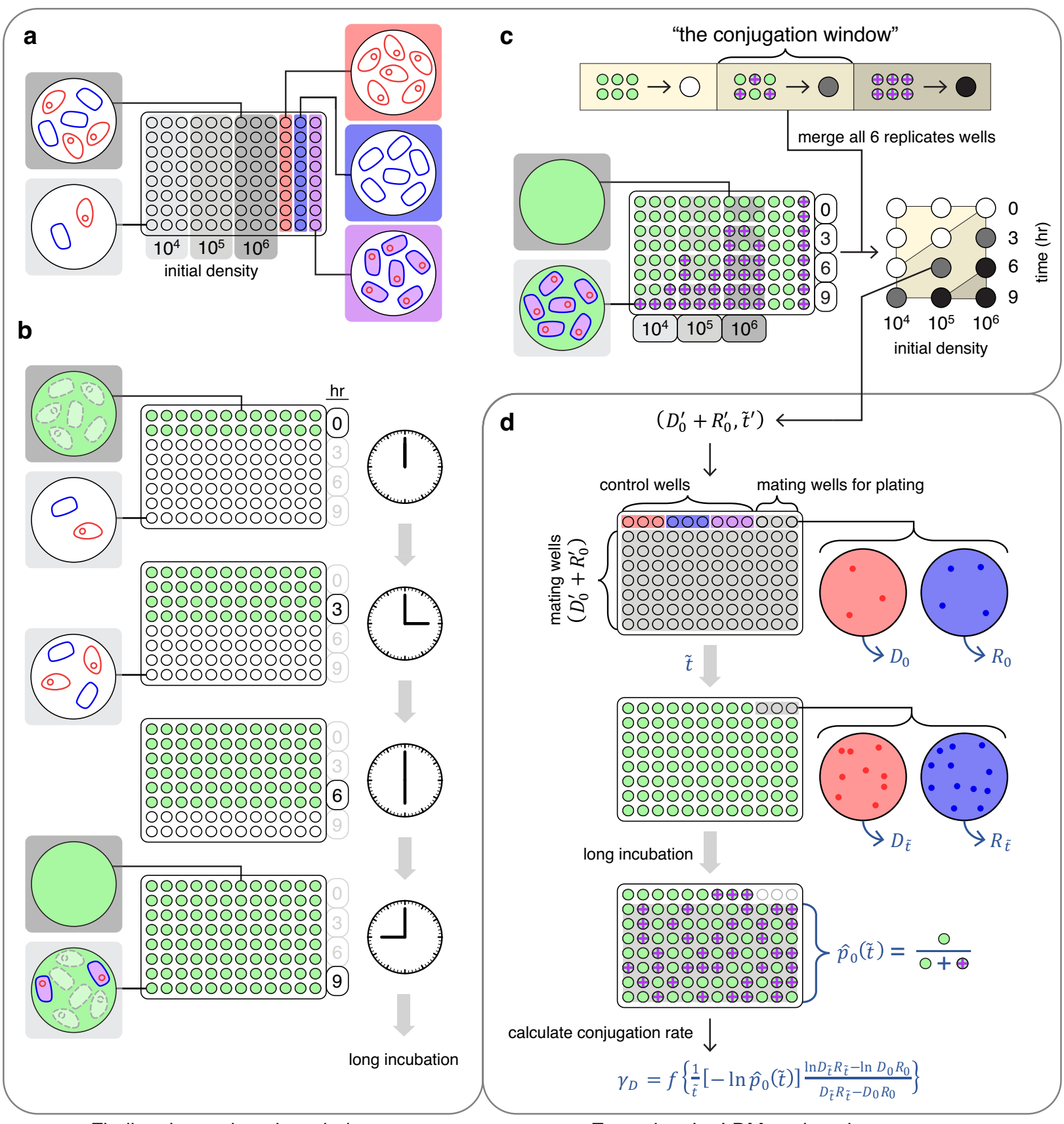

Finding the conjugation window

Executing the LDM conjugation assay

Figure 4 : Overview for finding the conjugation window (a-c) and executing the LDM conjugation assay (d). (a) Microtiter plate map designating placement of donors (red), recipients (blue), transconjugants (purple), and mating cultures over 10-fold dilutions (different shades of gray). For simplicity, donors and recipients are at the same proportion in each mating culture. The control wells are the last three columns. (b) Using the microtiter plate from part a, transconjugant-selecting medium (green well fill) is added at each time period designated by two rows in the microtiter plate. Two example wells from different time-density treatments are highlighted on the left where the gray background shading refers to the initial density treatment labeled in part a. In the top example well, transconjugant-selecting medium is added immediately, inhibiting growth of donor and recipient cells (grey dashed cells), and resulting in a non-turbid well as no transconjugants formed. In the bottom example well, the donor and recipient populations in the mating culture grow until transconjugant-selecting medium is added at 9-hours, inhibiting growth of donors and recipients, and permitting growth of the formed transconjugants. (c) After a lengthy incubation of the microtiter plate from part $b$, there are two well-types in the microtiter plate (bottom-left): transconjugant-containing (green well with purple cross) and 
transconjugant-free (green well). For each treatment (time-density pair), the 6 mating wells are considered as a group, resulting in one of three outcomes (top): all transconjugant-free wells (white dot), all transconjugant-containing wells (black dot), a proportion of both well-types (gray dot). Any treatment with a gray dot represents a viable combination of initial densities $\left(D_{0}^{\prime}+R_{0}^{\prime}\right)$ and incubation time $\left(\tilde{t}^{\prime}\right)$. (d) The microtiter plate is set up with many matings (gray) at the chosen target density $\left(D_{0}^{\prime}+R_{0}^{\prime}\right)$ in addition to control wells. Three matings are sampled to determine the actual initial densities $\left(D_{0}\right.$ and $\left.R_{0}\right)$ and final densities $\left(D_{\tilde{t}}\right.$ and $\left.R_{\tilde{t}}\right)$. After the incubation time $(\tilde{t})$, which is as close to the target incubation time $\left(\tilde{t}^{\prime}\right)$ as possible, transconjugant-selecting medium is added to the microtiter plate (excluding the matings used for density-plating). After a lengthy incubation, the proportion of transconjugant-free (i.e., non-turbid) wells is calculated, yielding $\hat{p}_{0}(\tilde{t})$. Using the actual incubation time $(\tilde{t})$, initial densities $\left(D_{0}, R_{0}\right)$, final densities $\left(D_{\tilde{t}}, R_{\tilde{t}}\right)$, and correcting for the experimental culture volume $(f)$, the LDM estimate is used to calculate donor conjugation rate $\left(\gamma_{D}\right)$. Part $d$ can be repeated to obtain replicate LDM estimates.

\section{Application of the LDM}

To explore the logistics of implementing the LDM, we measured the conjugation rate of a conjugative, IncP-1 $\beta$ plasmid (pALTS29) encoding chloramphenicol resistance in an Escherichia coli host ${ }^{4}$. Jordt et. al. ${ }^{4}$ used this plasmid-host pair as an ancestral strain which was propagated in chloramphenicol antibiotic for 400 generations to explore mechanisms of plasmid-host co-evolution. The evolved lineage acquired genetic modifications in the host chromosome and the plasmid. The ancestral plasmid was gradually lost from the ancestral host population when these plasmid-bearing cells were propagated under conditions that did not select for the plasmid (Figure 5a, dashed line). In contrast, the evolved plasmid was considerably more persistent in the absence of selection in its co-evolved host (Figure 5a, solid line).

This pattern of increased plasmid persistence after plasmid-host co-evolution is consistent with previous studies ${ }^{22,23}$. In most cases, the cost of plasmid carriage is assumed to decrease with plasmid-host co-evolution; however, changes in other factors, such as an increased rate of conjugation or a decreased rate of segregational loss, may observed increase in plasmid persistence in the Jordt. et. al. ${ }^{4}$ study is at least in part due to a significant increase in the conjugation rate in the evolved lineage (Figure 5b, MannWhitney $U$ test, $p$-value $=0.029$ ).

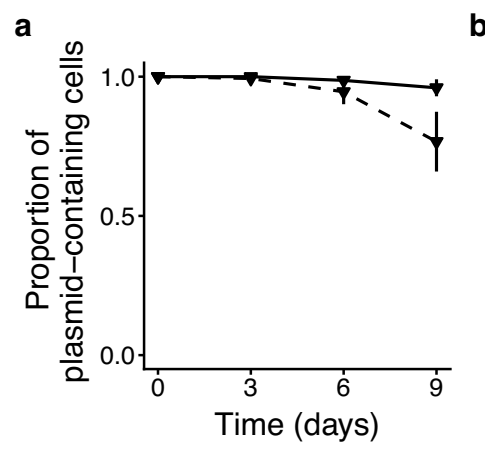

b

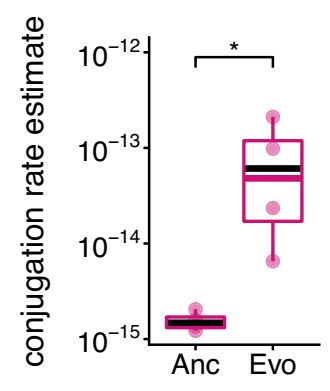


Figure 5 : LDM conjugation rate estimates of an ancestral and evolved lineage of $E$. coli containing a conjugative IncP-1 $\beta$ plasmid. (a) Plasmid persistence in the absence of chloramphenicol antibiotic was higher in the evolved plasmid-host pair (solid line) than the ancestral plasmid-host pair (dashed line). Data and figure adapted from Jordt et. al. ${ }^{4}$ (b) Conjugation rate significantly increased in the evolved (Evo) plasmid-host pair compared to the ancestral (Anc) plasmid-host pair. The points represent a conjugation rate estimate from an LDM conjugation assay. The black line is the mean of four replicates. The colored line is the median of the four replicates. The asterisk indicates a significant difference from a Mann-Whitney $\mathrm{U}$ test.

471

\section{Discussion}

Here we have presented a new method for estimating the rate of plasmid conjugative transfer from a donor cell to a recipient cell. We derived our LDM estimate using a mathematical approach that captures the stochastic process of conjugation, which was inspired by the method Luria and Delbrück applied to the process of mutation. This departs from the mathematical approach for other conjugation rate estimates which assume underlying deterministic frameworks guiding the dynamics of transconjugants ${ }^{11,13,16}$. One noteworthy conjugation assay from Johnson \& Kroer also embraced the stochastic nature of conjugation ${ }^{21}$. Similar to one aspect of our approach, they survey a series of mating cultures for the presence of transconjugants and use the fraction of transconjugant-free cultures to derive a conjugation metric. However, their metric yields the number of conjugation events and does not provide an estimate for the conjugation rate. Thus, the population dynamics of donors and recipients, as well as the incubation time, are not considered. The LDM captures positive features of both the Johnson \& Kroer approach and the deterministic-based approaches: specifically, it both recognizes the stochastic nature of conjugation and provides an analytic estimate for the conjugation rate.

Beyond the incorporation of stochasticity, the model and derivation behind the LDM estimate relaxes assumptions that constrained former approaches. This makes calculating conjugation rates accessible to a wide range of experimenters that use different plasmid-donor-recipient combinations. The LDM estimate makes no restrictive assumptions about growth rates or conjugation rates of different bacterial strains. Other conjugation rate estimates assume little to no change in the density due to growth (TDR), growth rates are identical across all strains (TDR and SIM), conjugation rates are identical for donors and transconjugants (SIM), or conjugation events from transconjugants are few (TDR and ASM)11,13,16. By relaxing these assumptions, we have increased the applicability of this new estimate, and our simulation results confirmed the high accuracy of the LDM under such scenarios. In contrast, the other conjugation methods (TDR, SIM, 
499 and ASM) sometimes yielded inaccurate estimates when their underlying assumptions were violated. Because violations of these assumptions are likely in natural plasmid systems, these performance differences could be important. For instance, unequal growth rates can occur among isogenic strains if the plasmid increases or decreases the growth rate of a host ${ }^{5,24}$. Also, growth rates may be significantly different when the donor and recipient are different strains or species. For example, some strains of Escherichia coli can divide every 20 minutes while some strains of Shewanella oneidensis only divide every 45 minutes ${ }^{25,26}$. Consequently, the growth rates of all the cell types will differ due to these combined effects in many plasmid transfer experiments. Such compounding effects could increase the error in measuring conjugation rate with the TDR and SIM estimates. Moreover, unequal growth rates are not likely to occur in isolation, but in tandem with other modeling violations such as unequal plasmid transfer rates. The consequences of such scenarios remain to be explored.

Perhaps the most significant advantage of our LDM method is the capability to accurately estimate the donor conjugation rate when it deviates from the transconjugant conjugation rate. For the previous methods, the error in estimating the donor conjugation rate increases as the transconjugant conjugation rate gets larger than the donor conjugation rate. These differences are likely to occur in plasmid transfer experiments. For instance, Dimitriu et. al. ${ }^{27}$ used the TDR approach to measure donor conjugation rate where the recipient was a clone-mate or a different species. The authors observed crossspecies conjugation rates that were up to 4 orders of magnitude lower than the withinspecies conjugation rates. If this pattern held generally, then a cross-species mating assay would violate a key assumption of previous methods, in that the transconjugant conjugation rate would be much higher than the donor conjugation rate. Indeed, the "unequal conjugation rates" simulations resulted in inflated conjugation estimates for the other methods (Figure $2 b$ ). For these reasons, reported cross-species conjugation rates may also be overestimates. In any case, unequal conjugation rates are likely, especially in microbial communities with diverse species. In addition, unequal conjugation rates can arise due to a molecular mechanism encoded on the conjugative plasmid, such as transitory de-repression ${ }^{13,28}$. In this case, a conjugation event between donor and recipient will temporarily elevate the conjugation rate of the newly formed transconjugant. This phenomenon would lead to similar outcomes as described above for different 
531 species. The LDM method can accurately estimate donor conjugation rates in systems with unequal conjugation rates, whether the differences are taxonomic or molecular in origin.

The LDM estimate also has advantages in terms of precision. Since conjugation is a stochastic process, the number of transconjugants at any given time is a random variable with a certain distribution. Therefore, estimates that rely on the number of transconjugants (TDR, SIM and ASM) or the probability of their absence (LDM) will also fall into distributions (Figure 2). Even in cases where the mean (first moment of the distribution) is close to the actual conjugation rate, the variance (second central moment) may differ among estimates. In fact, the LDM estimate had smaller variance compared to other estimates, even under parameter settings where different estimates shared similar accuracy (Figure 2). This greater precision likely originates from the difference in the distribution of the number of transconjugants $\left(T_{\tilde{t}}\right)$ and the distribution of the probability of transconjugant absence $\left(p_{0}(\tilde{t})\right)$, something we explore analytically in SI section 14 . Beyond the mean and variance, other features of these distributions (i.e., moments) may also be important. For certain parameter settings, the estimates relying on transconjugant numbers were asymmetric (the third moment was non-zero). In such cases, a small number of replicate estimates could lead to bias. For example, when there is a strong growth benefit to carrying the plasmid, the distribution for the ASM estimate is skewed even though the mean of this distribution reflects the actual conjugation rate (see Figure 2a). Typically a small number of conjugation assays is often the standard in studies; thus, the general position and shape of these estimate distributions may matter. Over the portion of parameter space we explored, the LDM distribution facilitated accurate and precise estimates through its position (a mean reflecting the true value) and its shape (a small variance and a low skew).

For all the methods considered, the distribution of conjugation estimates changed with time (Figure 3). Therefore, the incubation time becomes an important parameter. In our simulations, the estimate distribution generally narrowed with longer incubation times (Figure 3). This would suggest that one advantage of longer incubation is greater precision. However, when underlying assumptions are violated, there can be an interesting interaction between accuracy and precision over time. For instance, consider the case where transconjugant conjugation rate exceeds donor conjugation rate (Figure 
563 3b). For the SIM and ASM metrics, as incubation time rises, there is a shift from an accurate imprecise estimate to an inaccurate precise estimate. For an assay with a limited number of replicates in such a case, the optimal incubation time may be intermediate (a topic worthy of formal investigation). Over a range of incubation times where $p_{0}(\tilde{t})$ is not close to 0 or 1 , the LDM distribution is generally immune to these problems, exhibiting constant high accuracy and precision over time (Figure 3).

To execute a conjugation assay using any of the methods described, the experimenter needs to make several decisions regarding various protocol features such

\section{3} as incubation time and density of the mating culture. All of these choices need to conform

\section{5} closely to the modeling assumptions of each method and ideally maximize the accuracy of the conjugation rate estimate. For instance, the TDR assay seems simpler than the other methods, since it only requires measurement of the final densities of cells. However, implementing the TDR assay can be difficult because it assumes little to no change in density due to growth. For a growth-based TDR implementation, a very short incubation time could limit cell growth, but the density of the culture may need to be very high so transconjugants can form during the assay. Previous studies have shown that for many plasmids the conjugation rate is maximal during exponential phase ${ }^{13,17,29}$; therefore, using high density culture may underestimate the conjugation rate. A chemostat implementation could circumvent some of these issues by allowing lower density culture, exponential growth, and longer incubation times. However, unequal growth rates in a chemostat would lead to changes in the density of the donors and recipients. For the SIM approach, a standard growth-based 24-hour conjugation assay is often used5,30,31,32,33. This serves as an easy default choice and has many laboratory benefits for efficiency. Even so, the 24-hour incubation involves more than initial and final sampling, as two additional samplings are required to measure the maximum population growth rate during exponential phase (SI section 2). If these additional samplings are forgotten for the SIM approach, highly inaccurate estimates of the conjugation rate can result. In addition, the underlying SIM assumptions of equal growth rates and conjugation rates are easy to violate, and inaccuracies due to such violations can grow with incubation time (Figure 3b). For the ASM, a standard incubation time is not recommended; rather, this time is left to the experimenter to determine. However, the authors suggest short incubation times to prevent biased estimates if modeling assumptions are violated (e.g., relatively high 
595 transconjugant transfer rates). The authors mention that a follow-up assay to measure the transconjugant transfer rate may be needed to determine if the incubation time was short enough to prevent deviations. In contrast, the LDM protocol is designed to lead the experimenter to valid incubation times by explicitly identifying the conjugation window where $p_{0}(\tilde{t})$ can be estimated. Therefore, the experimenter can be sure the protocol occurred during a usable incubation time if the assay results in a $\hat{p}_{0}(\tilde{t})$ between 0 and 1 . We note that identifying the conjugation window does add an extra step in the LDM approach; however, a similar assay could help an experimenter choose an optimal incubation time for the other assays. Even so, the effects of these implementation choices on the accuracy and precision of conjugation rate estimates are currently understudied.

The LDM protocol offers an additional advantage by eliminating plating of the transconjugants. For the other methods, this is typically a source of error since the transconjugant density is often determined by plating the mating culture on transconjugant selecting medium. With short culture incubation times before plating, which will increase the accuracy of the other methods, the transconjugant density is extremely low compared to the donor and recipient densities. Therefore, plating high density cultures is needed to be able to detect the transconjugants. This can lead to conjugation continuing on the agar plates, which produces extra transconjugant colonies that did not result from conjugation events before plating,15,34. Such 'matings on transconjugant-selecting agar plates' can occur before the donors and recipients are completely inhibited by the transconjugant-selecting agent (typically an antibiotic). Such

\section{2} events will lead to an inflation of the transconjugant density and thus inaccuracy in the conjugation rate estimate. We note that the LDM avoids this problem since it relies only on recording transconjugant presence/absence in the liquid mating cultures. to estimate the conjugation rate for an IncP-1 $\beta$ plasmid in an $E$. coli host. Interestingly, we observed the evolution of conjugation rate. This comes as a bit of a surprise given that the environment in which the $E$. coli evolved contained an antibiotic selecting for plasmid carriage. Thus, plasmid-free cells would have been rare leading to few opportunities for plasmid conjugation into such cells. Bacterial cells containing a conjugative plasmid in a community with an abundance of plasmid-free cells is the scenario in which conjugation rates are expected to and have been observed to evolve ${ }^{35}$. 
627 Therefore, it is not immediately obvious what mechanism led to the increase in the 628 conjugation rate in our system. It is possible that increased conjugation rate could have been a pleiotropic effect from a different trait under direct selection. Alternatively, higher conjugation rate could evolve if (1) a mutation on the plasmid led to a higher transfer rate and (2) if donor cells can conjugate with other donors in our systems ${ }^{36}$. In such a case, the mutated plasmid would transfer quickly even under plasmid-selecting conditions and it could spread through the population as long as any associated costs (e.g., lower growth of its host or lower replication efficiency within mixed-plasmid hosts) were sufficiently low. This hypothetical sequence of events could lead to an increased conjugation rate. Regardless of the mechanism, it is interesting that bacterial populations in environments containing an antibiotic could lead to the evolution of higher conjugation rates of plasmids encoding resistance to that antibiotic. Importantly, such evolution could lead to higher frequency of antibiotic resistance in microbial communities, even in the absence of the antibiotic, due to more plasmid conjugation events.

\section{8}

In conclusion, the LDM offers new possibilities for measuring the conjugation rate that we did not fully review all the conjugation assays currently in use in the literature ${ }^{15,37}$, focusing instead on the least laborious and most widely used methods. Importantly, the LDM eliminates the possible bias caused by high transconjugant conjugation rates. Due to the sensitivity of the LDM conjugation assay and the ease of implementation, we were able to show the evolution of conjugation rate for an IncP-1 $\beta$ conjugative plasmid in an Escherichia coli host. However, the LDM method can be applied even more broadly given the flexibility of the underlying theoretical framework and the relative ease of assay implementation.

\section{Acknowledgements}

This work is supported by the National Institute of Allergy and Infectious Diseases Extramural Activities grant no. R01 Al084918 of the National Institutes of Health. O.K. is supported by the NSF Graduate Research Fellowship grant no. DGE-1762114. C.E. is supported by the NSF Graduate Research Fellowship. We thank Hannah Jordt, Simon 
658 Snoeck, and members of the Kerr and Top laboratories for useful suggestions on the 659 manuscript.

\section{Supplemental Information}

\section{SI section 1 : Historical theoretical perspective}

In this section, we highlight three key methods for estimating conjugation rate. While outlining the theoretical framework, we highlight the key distinctions and theoretical assumptions of each approach. Levin et. al. ${ }^{13}$ introduced a simple mathematical model for plasmid population dynamics to isolate the conjugation rate parameter $(\gamma)^{13}$. In their model, the donor population grows exponentially at a rate $\psi$ (equation [1]). The number of transconjugants increases due to conjugation events ( $2^{\text {nd }}$ term equation [3]) by donors and by existing transconjugants as well as exponential growth of the existing transconjugant pool ( $1^{\text {st }}$ term equation [3]). The recipient population grows exponentially at a rate $\psi$ and is depleted at the rate that new transconjugants form due to conjugation events ( $2^{\text {nd }}$ term equation [2]). For ease of reference, model variables and parameters are in SI Table 1 (the LDM is included for a complete comparison).

SI Table 1: Variables and parameters used in plasmid dynamic models 
bioRxiv preprint doi: https://doi.org/10.1101/2021.01.06.425583; this version posted December 3, 2021. The copyright holder for this preprint (which was not certified by peer review) is the author/funder, who has granted bioRxiv a license to display the preprint in perpetuity. It is made available under aCC-BY-NC-ND 4.0 International license.

\begin{tabular}{|c|c|c|c|}
\hline Variable/Parameter & Description & Relevant Estimate & Units \\
\hline$D$ & Donor density & TDR, SIM, ASM, LDM & \multirow{3}{*}{$\frac{\mathrm{cfu}}{\mathrm{ml}}$} \\
\hline$R$ & Recipient density & TDR, SIM, ASM, LDM & \\
\hline$T$ & Transconjugant density & TDR, SIM, ASM, LDM & \\
\hline$\psi$ & Growth rate (not population specific) & TDR, SIM & \multirow{4}{*}{$\frac{1}{h}$} \\
\hline$\psi_{D}$ & Donor growth rate & ASM, LDM & \\
\hline$\psi_{R}$ & Recipient growth rate & ASM, LDM & \\
\hline$\psi_{T}$ & Transconjugant growth rate & ASM, LDM & \\
\hline$\gamma$ & Conjugation rate (not population specific) & TDR, SIM & \multirow{3}{*}{$\frac{\mathrm{ml}}{\mathrm{cfu} \cdot \mathrm{h}}$} \\
\hline$\gamma_{D}$ & Donor-recipient conjugation rate & ASM, LDM & \\
\hline$\gamma_{T}$ & Transconjugant-recipient conjugation rate & ASM, LDM & \\
\hline
\end{tabular}

673

674

675

676

677

678

679

680

681

682

683

684

685

686

687

688

689

690

691

692

Equations [1] - [3] contains four notable assumptions. First, conjugation is described by mass-action kinetics, where conjugation events are proportional to the product of donor and recipient cell densities, which is a reasonable assumption in wellmixed liquid cultures ${ }^{13}$. Second, the model assumes a negligible rate of segregation, a process whereby a dividing plasmid-containing cell produces one plasmid-containing daughter cell and one plasmid-free daughter. These first two assumptions also exist in all of the other conjugation estimate methods we discuss. Third, the growth rate is the same for all cell types (donors, recipients, and transconjugants). Fourth, the plasmid conjugation rate is the same from donors to recipients as from transconjugants to recipients.

Levin et. al. ${ }^{13}$ used their plasmid dynamic model to derive an estimate for plasmid conjugation rate $(\gamma)$ by making three additional simplifying assumptions. First, the change in cell density of donors due to growth is assumed to be negligible. Likewise, the change in cell density of recipients due to growth and to conjugation (i.e., transformation into transconjugants) is assumed to be negligible. Finally, transconjugants are assumed to be rare in the population resulting in negligible growth of transconjugants and conjugation from transconjugants to recipients. Thus, the increase in transconjugants cell density is through plasmid conjugation from donors to recipients (i.e., $d T / d t=\gamma D R$ ). Using these simplifying assumptions, Levin et. al. ${ }^{13}$ solved for an expression of the conjugation rate $\gamma$ (equation [4]) in terms of the density of donors, recipients, and transconjugants $\left(D_{\tilde{t}}, R_{\tilde{t}}\right.$, 
and $T_{\tilde{t}}$, respectively) after a period of incubation $\tilde{t}$ (see SI section 3 for a few different approaches to this derivation). We label the expression in equation [4] as the "TDR" estimate for the conjugation rate, where the letters in this acronym come from the dynamic variables used in the estimate. TDR is a commonly used estimate ${ }^{14,13,15,17}$. However, the simplifying assumptions used to derive TDR, such as no change in density due to growth, can make implementation in the laboratory difficult. For ease of reference, all variables used in the conjugation estimates are in SI Table 2. All assumptions underlying each estimate are in SI Table 3. Both tables include LDM for a full comparison.

SI Table 2: Variables and parameters used to estimate* conjugation rate

\begin{tabular}{|c|c|c|c|}
\hline Variable/Parameter & Description & Relevant Estimate & Units \\
\hline$\tilde{t}$ & Incubation time (final sampling time) & TDR, SIM** ASM, LDM & $\mathrm{h}$ \\
\hline$D_{0}, R_{0}$ & Initial donor and recipient densities & ASM, LDM & \multirow{4}{*}{$\frac{\mathrm{cfu}}{\mathrm{ml}}$} \\
\hline$D_{\tilde{t}}, R_{\tilde{t}}$ & Final donor and recipient densities & TDR, SIM, ASM, LDM & \\
\hline$T_{\tilde{t}}$ & Final transconjugant density & TDR, SIM, ASM & \\
\hline$N_{0}, N_{\tilde{t}}$ & Initial and final total population density & SIM & \\
\hline$\psi_{T}$ & Transconjugant growth rate & ASM & $h^{-1}$ \\
\hline$p_{0}(\tilde{t})$ & $\begin{array}{l}\text { Probability a population has no } \\
\text { transconjugants }\end{array}$ & LDM & $\exp \left(\frac{\mathrm{cfu}}{\mathrm{ml}}\right)$ \\
\hline
\end{tabular}

\section{SI Table 3: Summary of modeling assumptions}

\begin{tabular}{|l|c|c|c|c|}
\hline Assumption & TDR & SIM & ASM & LDM \\
\hline Conjugation events follow mass-action kinetics & $\mathrm{X}$ & $\mathrm{X}$ & $\mathrm{X}$ & $\mathrm{X}$ \\
\hline There is no segregative loss of the plasmid & $\mathrm{X}$ & $\mathrm{X}$ & $\mathrm{X}$ & $\mathrm{X}$ \\
\hline The cell populations do not change in size due to growth & $\mathrm{X}$ & & & \\
\hline Processes of conjugation and growth are not resource dependent* & $\mathrm{X}$ & & $\mathrm{X}$ & $\mathrm{X}$ \\
\hline The cell populations grow exponentially & & & $\mathrm{X}$ & $\mathrm{X}$ \\
\hline $\begin{array}{l}\text { The growth rate is identical for all cell types } \\
\text { The transconjugant conjugation rate is not high relative to the donor } \\
\text { conjugation rate }\end{array}$ & $\mathrm{X}$ & $\mathrm{X}$ & $\mathrm{X}$ & $\mathrm{X}$ \\
\hline $\begin{array}{l}\text { * The SIM model is able to incorporate resource-dependent growth and conjugation because (1) growth } \\
\text { and transfer rates are homogeneous and (2) the functional form for resource dependence is the same } \\
\text { for growth and transfer. }\end{array}$ \\
\hline
\end{tabular}


In contrast to the assumption behind the TDR estimate, the model explored by Simonsen et. al. ${ }^{16}$ allows for population growth. Indeed, they allowed for the rate of population growth to change with the level of a resource in the environment, adding a dynamic variable for the resource concentration. In addition, the conjugation rate can also change with the resource concentration. The authors focus on a case where both growth and conjugation rates vary with resource concentration according to the Monod function. This choice was informed by experimental results showing that cells enter stationary phase and conjugation ramps down to a negligible level as resources are depleted ${ }^{13}$. This pattern occurs for various plasmid incompatibility groups, but not all ${ }^{29}$. Simonsen et. al. ${ }^{16}$ used this updated model to derive an estimate for plasmid conjugation rate (equation [5], see SI section 4 for the derivation). We term equation [5] as the "SIM" estimate for conjugation rate, where SIM stands for "Simonsen et. al. Identicality Method" since the underlying model assumes that all strains are identical with regards to growth, and donors and transconjugants are identical with regards to conjugation rate (as in equations [1][3]). The SIM estimate involves measuring the density of the initial population $\left(N_{0}\right)$, and the final density of donors $\left(D_{\tilde{t}}\right)$, recipients $\left(R_{\tilde{t}}\right)$, transconjugants $\left(T_{\tilde{t}}\right)$, and the total population $\left(N_{\tilde{t}}\right)$ after the incubation time $\tilde{t}$ (e.g., the end of an assay). The SIM estimate is a commonly used since it allows for the use of batch culture in the laboratory. Thus, it circumvents the constraints of the laboratory implementation of TDR; however, the underlying model (an enriched version of equations [1]-[3]) holds the same assumptions as before: homogeneous growth rates and conjugation rates.

\section{1}

Recently, Huisman et. al. ${ }^{11}$ updated the SIM model by introducing population specific growth rates for donors, recipients, and transconjugants $\left(\psi_{D}, \psi_{R}\right.$, and $\psi_{T}$, respectively) and population specific conjugation rates for donors and transconjugants $\left(\gamma_{D}\right.$ and $\left.\gamma_{T}\right)$. Importantly, the updated model is an approximation of the SIM due to three additional assumptions. First, conjugation and growth rates are assumed to be constant until resources are depleted, eliminating the additional resource concentration equation. Second, the increase in recipients due to growth greatly outpaces the decrease in recipients due to conjugation (i.e., $\psi_{R} R \gg \gamma_{D} D R+\gamma_{T} T R$ ). Third, the increase in transconjugants due to growth or plasmid conjugation from donors to recipients greatly outpaces the increase in transconjugants due to plasmid conjugation from transconjugants to recipients (i.e., $\psi_{T} T+\gamma_{D} D R \gg \gamma_{T} T R$ ). These model conditions are 
reasonable if the system starts with donors and recipients present but transconjugants are absent, the system is tracked for a short period of time $\tilde{t}$, conjugation rates are low relative to growth rates, and the transconjugant conjugation rate $\left(\gamma_{T}\right)$ is not much higher than the donor conjugation rate $\left(\gamma_{D}\right)$. With these added assumptions, equations [1]-[3] can be reformulated as the following approximate system of equations:

$$
\begin{gathered}
\frac{d D}{d t}=\psi_{D} D, \\
\frac{d R}{d t}=\psi_{R} R, \\
\frac{d T}{d t}=\psi_{T} T+\gamma_{D} D R,
\end{gathered}
$$

Huisman et. al. use this model to derive an estimate for conjugation rate (equation [6]) where different cell types now can have different growth rates (see SI section 5 for the derivation). We term equation [6] as the ASM estimate for donor conjugation rate, where ASM stands for "Approximate Simonsen et. al. Method". estimates: TDR, SIM, and ASM. Each method is explained either as recommended by its authors or the most simplified protocol to acquire the information for the estimate. For each, we describe proper laboratory implementation for the approaches based on the model and derivation assumptions used to acquire the estimate. Note in this section, we

\section{SI section 2 : Comparison of laboratory implementations}

In this section, we compare the laboratory implementations for various end-point do not explore the assumptions that are violated due to the biological samples being tested (i.e., specific species or plasmids) which can result in violations such as unequal conjugation rates or growth rates. These are explored in the main text via stochastic simulations. Thus, we focus solely on the parameters under the experimenter's control. For ease of reference, key implementation differences are highlighted in SI Table 4 (LDM is included for a full comparison).

SI Table 4: Comparison of implementations

\begin{tabular}{|l|c|c|c|c|}
\hline Summary & TDR & SIM & ASM & LDM \\
\hline Assay conditions minimizing the change in density due to growth & $\mathrm{X}$ & & & \\
\hline Minimize incubation time necessary for producing transconjugants & & & $\mathrm{X}$ & \\
\hline
\end{tabular}



available under aCC-BY-NC-ND 4.0 International license.

\begin{tabular}{|c|c|c|c|c|}
\hline $\begin{array}{l}\text { An incubation time results in a subset of parallel populations having no } \\
\text { transconjugants }\end{array}$ & & & & $\mathrm{X}$ \\
\hline Assay occurs over a period of exponential cell growth & & $X^{*}$ & $x$ & $\mathrm{X}$ \\
\hline Assay requires multiple parallel mating cultures per replicate & & & & $\mathrm{X}$ \\
\hline Assay requires a measurement of transconjugant density & $\mathrm{X}$ & $\mathrm{X}$ & $\mathrm{X}$ & \\
\hline Assay requires a measurement of population growth rate & & $X^{*}$ & & \\
\hline Assay requires a measurement of transconjugant growth rate & & & $X$ & \\
\hline
\end{tabular}

${ }^{*}$ For the SIM assay, either the entire assay is conducted over exponentially growing cultures or an independent estimate for (maximum) population growth rate is needed.

The TDR estimate has a simple form (equation [4]). Donors and recipients are mixed in non-selective growth medium and incubated for a specified time $\tilde{t}$. Typically, densities after the incubation time are determined using selective plating. The derivation assumes the density in donors and recipients does not change which sets specific constraints on incubation. In the original study, Levin et. al. ${ }^{9}$, used a chemostat to keep the population constant. Other studies shorten the incubation time $\tilde{t}$ such that population growth is negligible and use various laboratory tools to detect the small number of transconjugants ${ }^{15,17}$.

SIM circumvents the incubation limitations. Under conditions where the population is growing exponentially over the entire assay, we can rework the SIM estimate (equation [5]) into an alternative form for the laboratory (see SI section 6 for details).

$$
\gamma=\frac{1}{\tilde{t}}\left[\ln \left(1+\frac{T_{\tilde{t}}}{R_{\tilde{t}}} \frac{N_{\tilde{t}}}{D_{\tilde{t}}}\right)\right] \frac{\ln N_{\tilde{t}}-\ln N_{0}}{N_{\tilde{t}}-N_{0}}
$$

Donor and recipient populations in exponential phase are mixed in non-selective growth medium. The initial population density $\left(N_{0}\right)$ is determined by dilution plating on nonselective medium. After the mating mixture is incubated (for a period of $\tilde{t}$ ), the final densities $\left(D_{\tilde{t}}, R_{\tilde{t}}, T_{\tilde{t}}\right.$, and $\left.N_{\tilde{t}}\right)$ are determined by dilution plating on selective and nonselective media. To implement SIM as written in equation [2.1], the specified incubation time $\tilde{t}$ must occur well before stationary phase is reached to collect proper data for estimating the population growth rate $\left(\psi=\left(\ln N_{\tilde{t}}-\ln N_{0}\right) / \tilde{t}\right)$. There is an alternative option for implementing the SIM using equation [5]. The donor and recipient populations are mixed and incubated under batch culture conditions (including lag, exponential, and stationary phase). However, the (maximum) population growth rate $(\psi)$ needs to be determined with two additional samplings from the mixed population at times $t_{a}$ and $t_{b}$, both occurring within exponential phase: 


$$
\psi=\frac{\ln \left(N_{t_{b}} / N_{t_{a}}\right)}{t_{b}-t_{a}}
$$

The population densities $N_{t_{a}}$ and $N_{t_{b}}$ can be estimated either through colony counts from plating or optical density from a spectrophotometer. Either way, the timing of exponential phase is important for this approach and at least some analysis during this phase is required regardless of the implementation strategy.

For the ASM estimate, we can rework equation [6] into an alternate form for the laboratory (see SI section 6 for details).

$$
\gamma_{D}=\left\{\frac{1}{\tilde{t}}\left(\ln D_{\tilde{t}} R_{\tilde{t}}-\ln D_{0} R_{0}\right)-\psi_{T}\right\} \frac{T_{\tilde{t}}}{\left(D_{\tilde{t}} R_{\tilde{t}}-D_{0} R_{0} e^{\psi_{T} \tilde{t}}\right)}
$$

Donor and recipient populations in exponential phase are mixed in non-selective medium. Initial densities $\left(D_{0}\right.$ and $\left.R_{0}\right)$ are determined by plating dilutions on the appropriate selective media. After donor and recipient incubate $(\tilde{t})$, final densities $\left(D_{\tilde{t}}, R_{\tilde{t}}\right.$, and $\left.T_{\tilde{t}}\right)$ are determined by plating dilutions on the appropriate selective media. From the transconjugant-selecting agar plates, a transconjugant clone is incubated in monoculture then sampled twice (at times $t_{a}$ and $t_{b}$ ) in exponential phase to measure the transconjugant growth rate $\left(\psi_{T}=\ln \left(T_{t_{b}} / T_{t_{a}}\right) /\left(t_{b}-t_{a}\right)\right)$. The authors point out a critical consideration for proper implementation of the ASM is the incubation time $\tilde{t}$. Not only is sampling in exponential phase important, but if the incubation time $\tilde{t}$ is too long and passes a critical time $\left(t_{\text {crit }}\right)$ the approximations used to derive the ASM break down. To avoid overshooting $t_{c r i t}$, the authors recommend sampling as soon as measurable transconjugants arise. To determine that the incubation time used was below the critical time $\left(t_{\text {crit }}\right)$, a second assay is recommended by the authors to measure the transconjugant conjugation rate $\gamma_{T}$, which will determine if the original incubation time $\tilde{t}$ was below $t_{c r i t}$ for measuring the donor conjugation rate. This second assay would have the transconjugant clone become the donor in the mixture, while a newly marked recipient must be used so that donors and recipients can be distinguished using selective plating.

Each method has aspects of implementation in common. Each one shares the basic approach of mixing donors and recipients over some incubation time $\tilde{t}$. Each estimate requires reliable selectable markers to differentiate donors, recipients, and transconjugants. However, all estimates have some constraints on initial densities and time of measurement. This can occur because the experimenter needs to capture conjugation events (all estimates require this), avoid population growth (TDR), or keep 
growth exponential (ASM, and at least parts of SIM). Even so, each method has clear distinctions. The most notable is the incubation time $\tilde{t}$ (i.e., the end of the assay). The TDR method is constrained to conditions where no change in population size due to growth can occur. For SIM, initial and final sampling are not constrained to a particular phase of growth; however, measurement of the growth rate must occur during the exponential growth phase. For ASM, initial sampling is in early exponential phase, and the final sampling needs to occur during a specific time window. In other words, the assay needs to be long enough that measurable transconjugants appear, but short enough so that assumptions aren't violated (which can occur if transconjugant density becomes too large).

\section{5}

\section{SI section 3 : TDR derivation}

We present a few derivations for the TDR method. The derivations differ by the assumptions used to solve for TDR. We use this section to show all of the conditions where the TDR method can be used. In addition, we use this section to walk through all of the mathematical steps and provide an easy reference for comparing the derivations of all four methods (TDR, SIM, ASM, and LDM). The original condition presented by Levin et. al. ${ }^{13}$ is the last derivation described in this section.

Here we assume that we are considering conditions where population growth is not occurring. These conditions could involve an environment hindering cell division. Alternatively, the environment could promote growth, but we restrict the time period sufficiently so that population change is negligible. Or finally, if all strains possess the same growth rate, the population could be growing over longer periods of time but doing so in a continuous flow-through device (e.g., a chemostat) such that population density remains constant (see Levin et. al. ${ }^{13}$ ). Because we assume no segregative loss of the plasmid, the donor population must remain constant

$$
D_{t}=D_{0}
$$

for any time $t$ under consideration. Importantly, even though growth is not occurring, conjugation can proceed. We assume $T_{0}=0$, but the population of transconjugants can increase over time. Because every transconjugant was formerly a recipient cell, it must be the case that 
for any time $t$ under consideration. Therefore,

$$
R_{t}=R_{0}-T_{t}
$$

The dynamics of transconjugants is given by

$$
\frac{d T_{t}}{d t}=\gamma_{D} D_{t} R_{t}+\gamma_{T} T_{t} R_{t}
$$

840

841

842

843

844

845

846

847

848

849

850

851

852

853

52

By substituting terms from equations [3.1] and [3.2]

$$
\begin{gathered}
\frac{d T_{t}}{d t}=\gamma_{D} D_{0}\left(R_{0}-T_{t}\right)+\gamma_{T} T_{t}\left(R_{0}-T_{t}\right), \\
\frac{d T_{t}}{d t}=\left(\gamma_{D} D_{0}+\gamma_{T} T_{t}\right)\left(R_{0}-T_{t}\right), \\
\frac{d T_{t}}{d t}=-\gamma_{T}\left(T_{t}+\frac{\gamma_{D} D_{0}}{\gamma_{T}}\right)\left(T_{t}-R_{0}\right),
\end{gathered}
$$

We can solve this differential equation by a separation of variables:

$$
\int_{0}^{\tilde{t}} \frac{d T_{t}}{-\gamma_{T}\left(T_{t}+\frac{\gamma_{D} D_{0}}{\gamma_{T}}\right)\left(T_{t}-R_{0}\right)}=\int_{0}^{\tilde{t}} d t .
$$

The following identity is relevant here:

$$
\frac{d\left\{\frac{1}{a(b-c)} \ln \frac{x-b}{x-c}\right\}}{d x}=\frac{1}{a(x-b)(x-c)} .
$$

Letting $x=T_{t}, a=-\gamma_{T}, b=-\frac{\gamma_{D} D_{0}}{\gamma_{T}}$, and $c=R_{0}$, we can proceed as follows:

$$
\begin{gathered}
\left.\left\{\frac{1}{\gamma_{T}\left(\frac{\gamma_{D} D_{0}}{\gamma_{T}}+R_{0}\right)} \ln \frac{T_{t}+\frac{\gamma_{D} D_{0}}{\gamma_{T}}}{T_{t}-R_{0}}\right\}\right|_{0} ^{\tilde{t}}=\left.(t)\right|_{0} ^{\tilde{t}}, \\
\frac{1}{\gamma_{T}\left(\frac{\gamma_{D} D_{0}}{\gamma_{T}}+R_{0}\right)}\left(\ln \frac{T_{\tilde{t}}+\frac{\gamma_{D} D_{0}}{\gamma_{T}}}{T_{\tilde{t}}-R_{0}}-\ln \frac{T_{0}+\frac{\gamma_{D} D_{0}}{\gamma_{T}}}{T_{0}-R_{0}}\right)=\tilde{t} .
\end{gathered}
$$

Because $T_{0}=0$

$$
\begin{gathered}
\frac{1}{\gamma_{T}\left(\frac{\gamma_{D} D_{0}}{\gamma_{T}}+R_{0}\right)}\left(\ln \frac{T_{\tilde{t}}+\frac{\gamma_{D} D_{0}}{\gamma_{T}}}{T_{\tilde{t}}-R_{0}}-\ln \frac{\frac{\gamma_{D} D_{0}}{\gamma_{T}}}{-R_{0}}\right)=\tilde{t} \\
\frac{1}{\gamma_{T}\left(\frac{\gamma_{D} D_{0}}{\gamma_{T}}+R_{0}\right)}\left(\ln \frac{-R_{0}\left(T_{\tilde{t}}+\frac{\gamma_{D} D_{0}}{\gamma_{T}}\right)}{\frac{\gamma_{D} D_{0}}{\gamma_{T}}\left(T_{\tilde{t}}-R_{0}\right)}\right)=\tilde{t}
\end{gathered}
$$




$$
\ln \frac{1+\frac{\gamma_{T} T_{\tilde{t}}}{\gamma_{D} D_{0}}}{1-\frac{T_{\tilde{t}}}{R_{0}}}=\left(\gamma_{D} D_{0}+\gamma_{T} R_{0}\right) \tilde{t}
$$

$$
\frac{1+\frac{\gamma_{T} T_{\tilde{t}}}{\gamma_{D} D_{0}}}{1-\frac{T_{\tilde{t}}}{R_{0}}}=\exp \left\{\left(\gamma_{D} D_{0}+\gamma_{T} R_{0}\right) \tilde{t}\right\}
$$

$$
1+\frac{\gamma_{T} T_{\tilde{t}}}{\gamma_{D} D_{0}}=\left(1-\frac{T_{\tilde{t}}}{R_{0}}\right) \exp \left\{\left(\gamma_{D} D_{0}+\gamma_{T} R_{0}\right) \tilde{t}\right\}
$$

$$
\frac{\gamma_{T} T_{\tilde{t}}}{\gamma_{D} D_{0}}+\frac{T_{\tilde{t}}}{R_{0}} \exp \left\{\left(\gamma_{D} D_{0}+\gamma_{T} R_{0}\right) \tilde{t}\right\}=\exp \left\{\left(\gamma_{D} D_{0}+\gamma_{T} R_{0}\right) \tilde{t}\right\}-1
$$

$$
\begin{gathered}
T_{\tilde{t}}\left[\frac{\gamma_{T}}{\gamma_{D} D_{0}}+\frac{1}{R_{0}} \exp \left\{\left(\gamma_{D} D_{0}+\gamma_{T} R_{0}\right) \tilde{t}\right\}\right]=\exp \left\{\left(\gamma_{D} D_{0}+\gamma_{T} R_{0}\right) \tilde{t}\right\}-1, \\
T_{\tilde{t}}=\frac{R_{0}\left(\exp \left\{\left(\gamma_{D} D_{0}+\gamma_{T} R_{0}\right) \tilde{t}\right\}-1\right)}{\frac{\gamma_{T} R_{0}}{\gamma_{D} D_{0}}+\exp \left\{\left(\gamma_{D} D_{0}+\gamma_{T} R_{0}\right) \tilde{t}\right\}} .
\end{gathered}
$$

Thus, equation [3.5] is a general solution for the number of transconjugants at any time. If we assume that $\gamma_{T}=0$, then we can rewrite [3.4] as

$$
\ln \frac{1}{1-\frac{T_{\tilde{t}}}{R_{0}}}=\gamma_{D} D_{0} \tilde{t}
$$

$$
\begin{gathered}
-\ln \left(1-\frac{T_{\tilde{t}}}{R_{0}}\right)=\gamma_{D} D_{0} \tilde{t} \\
\gamma_{D}=\frac{-\ln \left(1-\frac{T_{\tilde{t}}}{R_{0}}\right)}{D_{0} \tilde{t}} .
\end{gathered}
$$

When $R_{0} \gg T_{\tilde{t}}$, a first-order Taylor approximation ensures $-\ln \left(1-\frac{T_{\tilde{t}}}{R_{0}}\right) \approx \frac{T_{\tilde{t}}}{R_{0}}$, and therefore

$$
\gamma_{D} \approx \frac{\frac{T_{\tilde{t}}}{R_{0}}}{D_{0} \tilde{t}}
$$

$$
\gamma_{D} \approx \frac{T_{\tilde{t}}}{D_{0} R_{0} \tilde{t}}
$$

Because $D_{\tilde{t}}=D_{0}$ (see [3.1]) and $R_{\tilde{t}} \approx R_{0}$ (when $R_{0} \gg T_{\tilde{t}}$ by [3.2]), we have

$$
\gamma_{D} \approx \frac{T_{\tilde{t}}}{D_{\tilde{t}} R_{\tilde{t}} \tilde{t}} .
$$

On the other hand, if we assume $\gamma_{D}=\gamma_{T}=\gamma$, then we can rewrite [3.4] as 


$$
\begin{gathered}
\ln \frac{1+\frac{T_{\tilde{t}}}{D_{0}}}{1-\frac{T_{\tilde{t}}}{R_{0}}}=\gamma\left(D_{0}+R_{0}\right) \tilde{t} \\
\gamma=\frac{1}{\tilde{t}\left(D_{0}+R_{0}\right)} \ln \frac{1+\frac{T_{\tilde{t}}}{D_{0}}}{1-\frac{T_{\tilde{t}}}{R_{0}}} \\
\gamma=\frac{1}{\tilde{t}\left(D_{0}+R_{0}\right)}\left\{\ln \left(1+\frac{T_{\tilde{t}}}{D_{0}}\right)-\ln \left(1-\frac{T_{\tilde{t}}}{R_{0}}\right)\right\} .
\end{gathered}
$$

871

872

873

874

875

876

877

878

879

880

881

882

883

884

885

886

887

888

889

When $D_{0} \gg T_{\tilde{t}}$ and $R_{0} \gg T_{\tilde{t}}$, first-order Taylor approximations ensure

$$
\begin{gathered}
\gamma \approx \frac{1}{\tilde{t}\left(D_{0}+R_{0}\right)}\left(\frac{T_{\tilde{t}}}{D_{0}}+\frac{T_{\tilde{t}}}{R_{0}}\right), \\
\gamma \approx \frac{T_{\tilde{t}}}{\tilde{t}\left(D_{0}+R_{0}\right)}\left(\frac{1}{D_{0}}+\frac{1}{R_{0}}\right), \\
\gamma \approx \frac{T_{\tilde{t}}}{\tilde{t}\left(D_{0}+R_{0}\right)}\left(\frac{R_{0}+D_{0}}{D_{0} R_{0}}\right), \\
\gamma \approx \frac{T_{\tilde{t}}}{D_{0} R_{0} \tilde{t}} .
\end{gathered}
$$

Because $D_{\tilde{t}}=D_{0}$ and $R_{\tilde{t}} \approx R_{0}$ (when $R_{0} \gg T_{\tilde{t}}$ ), we have

$$
\gamma \approx \frac{T_{\tilde{t}}}{D_{\tilde{t}} R_{\tilde{t}} \tilde{t}}
$$

So we have general expressions for donor conjugation rate when $\gamma_{T}=0$ (equation [3.6]) or when $\gamma_{D}=\gamma_{T}$ (equation [3.7]). However, when $D_{0} \gg T_{t}$ and $R_{0} \gg T_{t}$, for all $t$ under consideration, both of these measures are well approximated by equation [4]. Here we extend the application of equation [4] even further. When $R_{0} \gg T_{t}$, then $R_{t} \approx R_{0}$. Let us assume $R_{t}=R_{0}$. We will also assume

$$
\gamma_{D} D_{0} R_{0} \gg \gamma_{T} T_{t} R_{0}
$$

namely, the rate of formation of transconjugants by donors is much greater than the formation by transconjugants. Of course, if $\gamma_{T}=0$ or $\gamma_{D}=\gamma_{T}$, then $D_{0} \gg T_{t}$ ensures assumption [3.8]. However, assumption [3.8] does not require $\gamma_{T}=0$ or $\gamma_{D}=\gamma_{T}$ (indeed, assumption [3.8] is satisfied when $\gamma_{D} \geq \gamma_{T}$ and $D_{0} \gg T_{t}$, and $R_{0}>0$ ). In general, this inequality is satisfied when $T_{0}=0, D_{0}$ and $R_{0}$ are is large, $\gamma_{T}$ is not dramatically higher than $\gamma_{D}$, and the period is small. Under assumption [3.8], the dynamics can be well approximated by a simplified version of the differential equation [3.3] (where the 
transconjugant conjugation term is gone). This is the differential equation originally solved by Levin et. al.,

$$
\frac{d T_{t}}{d t}=\gamma_{D} D_{0} R_{0}
$$

Because everything on the right-hand-side of the equation is a constant, the solution is straightforward:

Because $T_{0}=0$,

\section{SI section 4 : SIM derivation}

$$
\begin{gathered}
\int_{0}^{\tilde{t}} d T_{t}=\int_{0}^{\tilde{t}} \gamma_{D} D_{0} R_{0} d t, \\
\left.\left(T_{\tilde{t}}\right)\right|_{0} ^{\tilde{t}}=\left.\left(\gamma_{D} D_{0} R_{0} t\right)\right|_{0} ^{\tilde{t}}, \\
T_{\tilde{t}}-T_{0}=\gamma_{D} D_{0} R_{0} \tilde{t} .
\end{gathered}
$$

$$
\text { Because } T_{0}=0
$$

SI section 4 : SIM derivation

Because $D_{\tilde{t}}=D_{0}$ and $R_{\tilde{t}}=R_{0}$ (by assumption), we have recovered equation [4].

$$
\begin{gathered}
T_{\tilde{t}}=\gamma_{D} D_{0} R_{0} \tilde{t}, \\
\gamma_{D}=\frac{T_{\tilde{t}}}{D_{0} R_{0} \tilde{t}} .
\end{gathered}
$$

In this section, we walk through all of the mathematical steps in the Simonsen et. al. derivation. We include this derivation as a quick reference to help the reader compare the derivations of all four methods (TDR, SIM, ASM, and LDM). Simonsen et. al. modified the model (equations [1]-[3]) by adding a dynamic variable for resource concentration $(C)$. The dynamics of this resource incorporate an additional parameter for the amount of resource needed to produce a new cell $(e)$. Both the growth rate and conjugation rate are taken to be functions of the resource concentration.

$$
\begin{gathered}
\frac{d D}{d t}=\psi(C) D \\
\frac{d R}{d t}=\psi(C) R-\gamma(C) R(D+T) \\
\frac{d T}{d t}=\psi(C) T+\gamma(C) R(D+T), \\
\frac{d C}{d t}=-\psi(C)(R+D+T) e .
\end{gathered}
$$

911 The other variables are consistent with their use in equations [1]-[3]. In Simonsen et. al. ${ }^{16}$, 912 when resources are depleted, growth and conjugation stop. A Monod function introduces 913 batch culture dynamics (i.e., exponential and stationary phase) making growth and 
914 conjugation both increase in a saturated manner with resource concentration, where the

915 resource concentration yielding $1 / 2$ the maximal rate is given by the parameter $Q$.

916 Importantly, conjugation and growth are assumed to have the same functional form:

where $Q$ is the half saturation constant.

Here we derive the estimation of conjugation rate from Simonsen et. al. ${ }^{16}$ focusing on the exponential phase of growth where resources are common ( $C$ is large) thus the following are reasonable assumptions:

$$
\begin{aligned}
& \psi(C)=\psi_{\max } \\
& \gamma(C)=\gamma_{\max }
\end{aligned}
$$

Under these assumptions, conjugation rate $(\gamma)$ and growth rate $(\psi)$ are constant. As resources are assumed to remain abundant such that there are no changes to the rates of growth and conjugation, equation [4.4] can be dropped. Simonsen et. al. ${ }^{16}$ assume that all strains have the same growth rate $\left(\psi_{D}=\psi_{R}=\psi_{T}=\psi_{\max }\right)$ and conjugation rates from donors and transconjugants are the same $\left(\gamma_{D}=\gamma_{T}=\gamma_{\max }\right)$. Additionally, Simonsen et. al. ${ }^{16}$ assume no segregative loss. Thus, with our assumption of exponential growth, we recover equations [1]-[3]. We define $N=D+R+T$. Therefore, using equations [1]-[3]

$$
\begin{gathered}
\frac{d N}{d t}=\frac{d D}{d t}+\frac{d R}{d t}+\frac{d T}{d t} \\
\frac{d N}{d t}=\psi_{\max } D+\psi_{\max } R-\gamma_{\max } R(D+T)+\psi_{\max } T+\gamma_{\max } R(D+T), \\
\frac{d N}{d t}=\psi_{\max }(D+R+T), \\
\frac{d N}{d t}=\psi_{\text {max }} N .
\end{gathered}
$$

The general solutions for equation [1] and [4.5] are found by (1) separating variables, (2) integrating, and (3) solving for $D_{t}$ or $N_{t}$, respectively.

$$
\begin{aligned}
& D_{t}=D_{0} e^{\psi_{\max } t}, \\
& N_{t}=N_{0} e^{\psi_{\max } t} .
\end{aligned}
$$

Define the fraction of donors in the population to be $X_{t}=D_{t} / N_{t}$. We note

$$
X_{t}=\frac{D_{t}}{N_{t}}=\frac{D_{0} e^{\psi_{\max } t}}{N_{0} e^{\psi_{\max } t}}=\frac{D_{0}}{N_{0}}=X_{0}
$$


941 Thus, this fraction does not change over time (i.e., $X_{t}$ is a constant). Lastly, we define a 942 fraction $Y_{t}=T_{t} / R_{t}$. Using equations [2] and [3],

$$
\frac{d Y}{d t}=\frac{\left\{\psi_{\max } T+\gamma_{\max } R(D+T)\right\} R-\left\{\psi_{\max } R-\gamma_{\max } R(D+T)\right\} T}{R^{2}}
$$

$$
\frac{d Y}{d t}=\frac{\psi_{\max } T R+\gamma_{\max }(D+T) R^{2}-\psi_{\max } T R+\gamma_{\max }(D+T) T R}{R^{2}}
$$

$$
\frac{d Y}{d t}=\frac{\gamma_{\max }(D+T) R^{2}+\gamma_{\max }(D+T) T R}{R^{2}},
$$

$$
\frac{d Y}{d t}=\frac{\gamma_{\max }(D+T) R+\gamma_{\max }(D+T) T}{R},
$$

Because $N=D+R+T$,

$$
\frac{d Y}{d t}=\frac{\gamma_{\max }\left(D R+T R+D T+T^{2}\right)}{R}
$$

$$
\frac{d Y}{d t}=\gamma_{\max } \frac{D T+R T+T^{2}+D R}{R}
$$

$$
\frac{d Y}{d t}=\gamma_{\max }\left(\frac{T(D+R+T)}{R}+D\right)
$$

$$
\frac{d Y}{d t}=\gamma_{\max }\left(\frac{T N}{R}+D\right)
$$$$
\frac{d Y}{d t}=\gamma_{\max } N\left(\frac{T}{R}+\frac{D}{N}\right)
$$

Because $X=D / N$ and $Y=T / R$,

$$
\frac{d Y}{d t}=\gamma_{\max } N(Y+X)
$$

$$
\left(\frac{1}{Y+X}\right) \frac{d Y}{d t}=\gamma_{\max } N
$$

Equation [4.5] ensures $N=\left(\frac{1}{\psi_{\max }}\right) \frac{d N}{d t}$, which yields

$$
\left(\frac{1}{Y+X}\right) \frac{d Y}{d t}=\gamma_{\max }\left(\frac{1}{\psi_{\max }}\right) \frac{d N}{d t},
$$

$$
\psi_{\max }\left(\frac{1}{Y+X}\right) \frac{d Y}{d t}=\gamma_{\max } \frac{d N}{d t}
$$

$$
\gamma_{\max }(d N)=\psi_{\max }\left(\frac{d Y}{Y+X}\right)
$$

To emphasize that $X$ is a constant, we write this as $X_{0}$ and then integrate both sides, 


$$
\gamma_{\max } \int_{0}^{\tilde{t}} d N=\psi_{\max } \int_{0}^{\tilde{t}}\left(\frac{d Y}{Y+X_{0}}\right),
$$

Making the time dependence of the variables explicit via subscripts

$$
\begin{gathered}
\left.\gamma_{\max } N_{t}\right|_{0} ^{\tilde{t}}=\left.\psi_{\max } \ln \left(Y_{t}+X_{0}\right)\right|_{0} ^{\tilde{t}}, \\
\gamma_{\max }\left(N_{\tilde{t}}-N_{0}\right)=\psi_{\max }\left\{\ln \left(Y_{\tilde{t}}+X_{0}\right)-\ln \left(Y_{0}+X_{0}\right)\right\} .
\end{gathered}
$$

966

Because $X_{0}=X_{\tilde{t}}$

$$
\begin{gathered}
\gamma_{\max }\left(N_{\tilde{t}}-N_{0}\right)=\psi_{\max }\left\{\ln \left(Y_{\tilde{t}}+X_{\tilde{t}}\right)-\ln \left(Y_{0}+X_{\tilde{t}}\right)\right\}, \\
\gamma_{\max }\left(N_{\tilde{t}}-N_{0}\right)=\psi_{\max } \ln \left(\frac{Y_{\tilde{t}}+X_{\tilde{t}}}{Y_{0}+X_{\tilde{t}}}\right) .
\end{gathered}
$$

Because $X_{\tilde{t}}=D_{\tilde{t}} / N_{\tilde{t}}$ and $Y_{\tilde{t}}=T_{\tilde{t}} / R_{\tilde{t}}$,

$$
\gamma_{\max }\left(N_{\tilde{t}}-N_{0}\right)=\psi_{\max } \ln \left(\frac{\frac{T_{\tilde{t}}}{R_{\tilde{t}}}+\frac{D_{\tilde{t}}}{N_{\tilde{t}}}}{\frac{T_{0}}{R_{0}}+\frac{D_{\tilde{t}}}{N_{\tilde{t}}}}\right) .
$$

Because $T_{0}=0$,

$$
\gamma_{\max }\left(N_{\tilde{t}}-N_{0}\right)=\psi_{\max } \ln \left(\frac{\frac{T_{\tilde{t}}}{R_{\tilde{t}}}+\frac{D_{\tilde{t}}}{N_{\tilde{t}}}}{\frac{D_{\tilde{t}}}{N_{\tilde{t}}}}\right)
$$

$$
\gamma_{\max }\left(N_{\tilde{t}}-N_{0}\right)=\psi_{\max } \ln \left(\frac{T_{\tilde{t}}}{R_{\tilde{t}}} \frac{N_{\tilde{t}}}{D_{\tilde{t}}}+1\right),
$$

$$
\gamma_{\max }=\psi_{\max } \ln \left(1+\frac{T_{\tilde{t}}}{R_{\tilde{t}}} \frac{N_{\tilde{t}}}{D_{\tilde{t}}}\right) \frac{1}{N_{\tilde{t}}-N_{0}} .
$$

Dropping the "max" subscripts, we have recovered equation [5].

\section{SI section 5 : ASM derivation}

In this section, we walk through all of the mathematical steps in the Huisman et. al. ${ }^{11}$ derivation. We include this derivation as a quick reference to help the reader compare the derivations of all four methods (TDR, SIM, ASM, and LDM). We start with a system of ordinary differential equations described by Huisman et. al. ${ }^{11}$, the Extended Simonsen Model (ESM):

$$
\begin{gathered}
\frac{d D}{d t}=\psi_{D}(C) D \\
\frac{d R}{d t}=\psi_{R}(C) R-\left(\gamma_{D}(C) D+\gamma_{T}(C) T\right) R
\end{gathered}
$$




$$
\begin{gathered}
\frac{d T}{d t}=\psi_{T}(C) T+\left(\gamma_{D}(C) D+\gamma_{T}(C) T\right) R, \\
\frac{d C}{d t}=-\left(\psi_{D}(C) D+\psi_{R}(C) R+\psi_{T}(C) T\right) e,
\end{gathered}
$$

Where $\psi$ subscripts specify population specific growth rates, $\gamma$ subscripts specify donor and transconjugant specific conjugation rates, and other variables are consistent with equations [1]-[3] or equations [4.1]-[4.4]. As with the SIM, growth and conjugation rate are both dependent on resource concentration. When resources are depleted, growth and conjugation stop.

where $A \in\{D, R, T\}$ and $B \in\{D, T\}$.

$$
\begin{aligned}
& \psi_{A}(C)=\frac{\psi_{A, \max } C}{Q+C}, \\
& \gamma_{B}(C)=\frac{\gamma_{B, \max C}}{Q+C},
\end{aligned}
$$

Under the assumption that growth rate and conjugation rate are constant (where $\psi_{A}(C)=\psi_{A, \max }$ and $\left.\gamma_{B}(C)=\gamma_{B, \max }\right)$, equation [5.4] can be dropped. Although the maximal rates of growth and conjugation are assumed, in what follows, we drop the "max" subscript for notational convenience. This new set of simplified ordinary differential equations is termed the Approximate Simonsen et. al. Method ('ASM').

$$
\begin{gathered}
\frac{d D}{d t}=\psi_{D} D, \\
\frac{d R}{d t}=\psi_{R} R-\left(\gamma_{D} D+\gamma_{T} T\right) R, \\
\frac{d T}{d t}=\psi_{T} T+\left(\gamma_{D} D+\gamma_{T} T\right) R,
\end{gathered}
$$

Under initial culture conditions, the final simple set of equations [1.1] - [1.3] arise by assuming the recipient population is dominated by growth $\psi_{R} R \gg\left(\gamma_{D} D+\gamma_{T} T\right) R$ and the transconjugant population is dominated by growth and conjugation from donors $\psi_{T} T+$ $\gamma_{D} D R \gg \gamma_{T} T R$. The solutions to differential equations [1.1] and [1.2] are:

$$
\begin{aligned}
& D_{t}=D_{0} e^{\psi_{D} t} \\
& R_{t}=R_{0} e^{\psi_{R} t}
\end{aligned}
$$

1000 Here we will derive the solution for the differential equation [1.3] for transconjugant density 1001 $T$. We know that $D_{t}=D_{0} e^{\psi_{D} t}$ and $R_{t}=R_{0} e^{\psi_{R} t}$, therefore 


$$
\frac{d T}{d t}=\psi_{T} T+\gamma_{D} D_{0} R_{0} e^{\left(\psi_{D}+\psi_{R}\right) t}
$$

We propose that the transconjugant density can be written as a product of time dependent functions:

$$
T_{t}=u_{t} v_{t}
$$

1004 We'll drop the $t$ subscripts for notational ease. By the product rule,

$$
\frac{d T}{d t}=u \frac{d v}{d t}+v \frac{d u}{d t}
$$

We can rewrite equation [5.10] by plugging in equations [5.11] and [5.12] as follows:

$$
\begin{aligned}
& u \frac{d v}{d t}+v \frac{d u}{d t}=\psi_{T} u v+\gamma_{D} D_{0} R_{0} e^{\left(\psi_{D}+\psi_{R}\right) t} \\
& u \frac{d v}{d t}+v\left(\frac{d u}{d t}-\psi_{T} u\right)=\gamma_{D} D_{0} R_{0} e^{\left(\psi_{D}+\psi_{R}\right) t}
\end{aligned}
$$

We have some freedom to pick $u_{t}$ as we please. So, let's choose a function such that the second term of the left-hand side of equation [5.13] is zero:

$$
\begin{gathered}
\frac{d u}{d t}-\psi_{T} u=0 \\
\frac{d u}{d t}=\psi_{T} u
\end{gathered}
$$

1011 The solution to this differential equation is:

$$
u_{t}=u_{0} e^{\psi_{T} t}
$$

1012 where $u_{0}$ is a constant.

1013 Thus, we can rewrite equation [5.13] as:

To solve this equation, we integrate

$$
\begin{gathered}
u_{0} e^{\psi_{T} t} \frac{d v}{d t}=\gamma_{D} D_{0} R_{0} e^{\left(\psi_{D}+\psi_{R}\right) t} \\
u_{0} \frac{d v}{d t}=\frac{\gamma_{D} D_{0} R_{0} e^{\left(\psi_{D}+\psi_{R}\right) t}}{e^{\psi_{T} t}} \\
u_{0} \frac{d v}{d t}=\gamma_{D} D_{0} R_{0} e^{\left(\psi_{D}+\psi_{R}\right) t} e^{-\psi_{T} t} \\
u_{0} \frac{d v}{d t}=\gamma_{D} D_{0} R_{0} e^{\left(\psi_{D}+\psi_{R}-\psi_{T}\right) t}
\end{gathered}
$$

1019

$$
u_{0} \int d v=\gamma_{D} D_{0} R_{0} \int e^{\left(\psi_{D}+\psi_{R}-\psi_{T}\right) t} d t
$$




$$
u_{0} v_{t}=\frac{\gamma_{D} D_{0} R_{0}}{\psi_{D}+\psi_{R}-\psi_{T}} e^{\left(\psi_{D}+\psi_{R}-\psi_{T}\right) t}+c
$$

where $c$ is a constant of the integration. To solve for $c$, plug in $t=0$,

$$
\begin{gathered}
u_{0} v_{0}=\frac{\gamma_{D} D_{0} R_{0}}{\psi_{D}+\psi_{R}-\psi_{T}} e^{\left(\psi_{D}+\psi_{R}-\psi_{T}\right) 0}+c \\
u_{0} v_{0}=\frac{\gamma_{D} D_{0} R_{0}}{\psi_{D}+\psi_{R}-\psi_{T}}+c \\
c=u_{0} v_{0}-\frac{\gamma_{D} D_{0} R_{0}}{\psi_{D}+\psi_{R}-\psi_{T}}
\end{gathered}
$$

1024 Because $T_{0}=u_{0} v_{0}$,

$$
c=T_{0}-\frac{\gamma_{D} D_{0} R_{0}}{\psi_{D}+\psi_{R}-\psi_{T}}
$$

So, we now can find the solution for $v_{\tilde{t}}$ by plugging in our solution for $c$ into equation

$$
\begin{gathered}
u_{0} v_{\tilde{t}}=\frac{\gamma_{D} D_{0} R_{0}}{\psi_{D}+\psi_{R}-\psi_{T}} e^{\left(\psi_{D}+\psi_{R}-\psi_{T}\right) \tilde{t}}+T_{0}-\frac{\gamma_{D} D_{0} R_{0}}{\psi_{D}+\psi_{R}-\psi_{T}} \\
u_{0} v_{\tilde{t}}=T_{0}+\frac{\gamma_{D} D_{0} R_{0}}{\psi_{D}+\psi_{R}-\psi_{T}}\left\{e^{\left(\psi_{D}+\psi_{R}-\psi_{T}\right) \tilde{t}}-1\right\} \\
v_{\tilde{t}}=\frac{1}{u_{0}}\left(T_{0}+\frac{\gamma_{D} D_{0} R_{0}}{\psi_{D}+\psi_{R}-\psi_{T}}\left\{e^{\left(\psi_{D}+\psi_{R}-\psi_{T}\right) \tilde{t}}-1\right\}\right)
\end{gathered}
$$

Because $T_{\tilde{t}}=u_{\tilde{t}} v_{\tilde{t}}$ through substitution of equations [5.14] and [5.16], we have

$$
\begin{gathered}
T_{\tilde{t}}=\left[u_{0} e^{\psi_{T} \tilde{t}}\right]\left[\frac{1}{u_{0}}\left(T_{0}+\frac{\gamma_{D} D_{0} R_{0}}{\psi_{D}+\psi_{R}-\psi_{T}}\left\{e^{\left(\psi_{D}+\psi_{R}-\psi_{T}\right) \tilde{t}}-1\right\}\right)\right] \\
T_{\tilde{t}}=e^{\psi_{T} \tilde{t}}\left(T_{0}+\frac{\gamma_{D} D_{0} R_{0}}{\psi_{D}+\psi_{R}-\psi_{T}}\left\{e^{\left(\psi_{D}+\psi_{R}-\psi_{T}\right) \tilde{t}}-1\right\}\right) \\
T_{\tilde{t}}=T_{0} e^{\psi_{T} \tilde{t}}+\frac{\gamma_{D} D_{0} R_{0}}{\psi_{D}+\psi_{R}-\psi_{T}}\left\{e^{\left(\psi_{D}+\psi_{R}\right) \tilde{t}}-e^{\psi_{T} \tilde{t}}\right\}
\end{gathered}
$$

Because $T_{0}=0$, we arrive at the result in Huisman et al. ${ }^{11}$

$$
T_{\tilde{t}}=\frac{\gamma_{D} D_{0} R_{0}}{\psi_{D}+\psi_{R}-\psi_{T}}\left\{e^{\left(\psi_{D}+\psi_{R}\right) \tilde{t}}-e^{\psi_{T} \tilde{t}}\right\}
$$

Given that $D_{\tilde{t}}=D_{0} e^{\psi_{D} \tilde{t}}$ and $R_{\tilde{t}}=R_{0} e^{\psi_{R} \tilde{t}}$, this can be rewritten as

$$
T_{\tilde{t}}=\frac{\gamma_{D}}{\psi_{D}+\psi_{R}-\psi_{T}}\left\{D_{\tilde{t}} R_{\tilde{t}}-D_{0} R_{0} e^{\psi_{T} \tilde{t}}\right\}
$$

Solving for $\gamma_{D}$ gives equation [6]. 


\section{SI section 6 : Alternative laboratory forms for conjugation estimates}

1041

To rearrange the SIM estimate, we start with equation [5]. If the entire period from $t=0$ to $t=\tilde{t}$ involves exponential growth, then $N_{\tilde{t}}=N_{0} e^{\psi \tilde{t}}$. In such a case, $\psi=$

To rearrange the ASM estimate, we start with equation [6]. While the equations $\psi_{D}=(1 / \tilde{t}) \ln \left(D_{\tilde{t}} / D_{0}\right)$ and $\psi_{R}=(1 / \tilde{t}) \ln \left(R_{\tilde{t}} / R_{0}\right)$ again provide estimates on donor and recipient growth rates, we cannot express the transconjugant growth rate $\left(\psi_{T}\right)$ as a simple expression of time and initial/final densities of members of the mating culture. However, data from a transconjugant monoculture supply an estimate on this parameter. Thus, we arrive at the laboratory form for ASM which is equation [2.3].

$$
\gamma_{D}=\left\{\frac{1}{\tilde{t}}\left(\ln D_{\tilde{t}} R_{\tilde{t}}-\ln D_{0} R_{0}\right)-\psi_{T}\right\} \frac{T_{\tilde{t}}}{\left(D_{\tilde{t}} R_{\tilde{t}}-D_{0} R_{0} e^{\psi_{T} \tilde{t}}\right)} .
$$

To rearrange the LDM estimate, we start with equation [13]. Since $D_{\tilde{t}}=D_{0} e^{\psi_{D} \tilde{t}}$ 1054 and $R_{\tilde{t}}=R_{0} e^{\psi_{R} \tilde{t}}$, it is the case that $\psi_{D}=(1 / \tilde{t}) \ln \left(D_{\tilde{t}} / D_{0}\right)$ and $\psi_{R}=(1 / \tilde{t}) \ln \left(R_{\tilde{t}} / R_{0}\right)$. So, we have

After rearrangement, we have

$$
\gamma_{D}=\frac{1}{\tilde{t}}\left\{-\ln p_{0}(\tilde{t})\right\} \frac{\ln \left(D_{\tilde{t}} R_{\tilde{t}}\right)-\ln \left(D_{0} R_{0}\right)}{D_{\tilde{t}} R_{\tilde{t}}-D_{0} R_{0}} .
$$

In the laboratory, we measure an estimate $\left(\hat{p}_{0}(\tilde{t})\right)$ of the probability that a population has no transconjugants $\left(p_{0}(\tilde{t})\right)$ which is simply the fraction of the populations (i.e., parallel cultures) that have no transconjugants at the incubation time $\tilde{t}$ (see SI section 9 for details). In addition, if a $1 \mathrm{ml}$ volume is not used for each mating culture (assuming that all cell densities are measured in $\frac{\mathrm{cfu}}{\mathrm{ml}}$ units), then we must add a correction factor $f$ which is the number of experimental volume units (evu) per $\mathrm{ml}$ (see SI section 10 for details and an example). Thus, we arrive at the laboratory form for the LDM which is equation [15].

$$
\gamma_{D}=f\left\{\frac{1}{\tilde{t}}\left[-\ln \hat{p}_{0}(\tilde{t})\right] \frac{\ln D_{\tilde{t}} R_{\tilde{t}}-\ln D_{0} R_{0}}{D_{\tilde{t}} R_{\tilde{t}}-D_{0} R_{0}}\right\}
$$


1068

1069

1070

1071

1072

1073

1074

1075

1076

1077

1078

1079

1080

1081

1082

1083

1084

1085

1086

1087

1088

1089

1090

1091

1092

1093

1094

\section{SI section 7 : Derivation of $p_{0}(\tilde{t})$ for the LDM estimate}

We define $p_{n}(t)$ to be the probability that there are $n$ transconjugants at time $t$, where $n$ is a non-negative integer (i.e., $p_{n}(t)=\operatorname{Pr}\left\{T_{t}=n\right\}$ ). We focus here on the probability that transconjugants are absent (namely, where $n=0$ ) and derive an expression for $p_{0}(t)$. By definition $p_{0}(t+\Delta t)=\operatorname{Pr}\left\{T_{t+\Delta t}=0\right\}$. However, $T_{t+\Delta t}=0$ implies $T_{t}=0$, so we can write

$$
p_{0}(t+\Delta t)=\operatorname{Pr}\left\{\left(T_{t+\Delta t}=0\right) \cap\left(T_{t}=0\right)\right\}=\operatorname{Pr}\left\{T_{t+\Delta t}=0 \mid T_{t}=0\right\} \operatorname{Pr}\left\{T_{t}=0\right\},
$$

Given that $p_{0}(t)=\operatorname{Pr}\left\{T_{t}=0\right\}$, we can use equation [11] to write the following timeincrement recursion for $p_{0}(t)$ :

$$
p_{0}(t+\Delta t)=\left(1-\gamma_{D} D_{t} R_{t} \Delta t\right) p_{0}(t) .
$$

This can be simplified as follows

$$
\frac{p_{0}(t+\Delta t)-p_{0}(t)}{\Delta t}=-\gamma_{D} D_{t} R_{t} p_{0}(t)
$$

Taking the limit as $\Delta t \rightarrow 0$ gives

$$
\lim _{\Delta t \rightarrow 0} \frac{p_{0}(t+\Delta t)-p_{0}(t)}{\Delta t}=\frac{d p_{0}(t)}{d t} .
$$

Therefore, we have the following differential equation:

$$
\frac{d p_{0}(t)}{d t}=-\gamma_{D} D_{t} R_{t} p_{0}(t)
$$

We are assuming $D_{t}=D_{0} e^{\psi_{D} t}$ and $R_{t}=R_{0} e^{\psi_{R} t}$. We note that these assumptions are reasonable if the densities of donors and recipients are reasonably large and the rate of transconjugant generation per recipient cell $\left(\gamma_{D} D_{t}+\gamma_{T} T_{t}\right.$, or if $T_{t}=0$, simply $\left.\gamma_{D} D_{t}\right)$ remains very small relative to per capita recipient growth rate. Under these assumptions, our differential equation becomes:

$$
\frac{d p_{0}(t)}{d t}=-\gamma_{D} D_{0} R_{0} e^{\left(\psi_{D}+\psi_{R}\right) t} p_{0}(t)
$$

We solve this differential equation via separation of variables, integrating from 0 to our incubation time of interest $\tilde{t}$ :

$$
\int_{0}^{\tilde{t}} \frac{d p_{0}(t)}{p_{0}(t)}=\int_{0}^{\tilde{t}}-\gamma_{D} D_{0} R_{0} e^{\left(\psi_{D}+\psi_{R}\right) t} d t
$$

$$
\left.\ln p_{0}(t)\right|_{0} ^{\tilde{t}}=\left.\frac{-\gamma_{D} D_{0} R_{0}}{\psi_{D}+\psi_{R}} e^{\left(\psi_{D}+\psi_{R}\right) t}\right|_{0} ^{\tilde{t}},
$$

$$
\ln p_{0}(\tilde{t})-\ln p_{0}(0)=\frac{-\gamma_{D} D_{0} R_{0}}{\psi_{D}+\psi_{R}} e^{\left(\psi_{D}+\psi_{R}\right) \tilde{t}}-\frac{-\gamma_{D} D_{0} R_{0}}{\psi_{D}+\psi_{R}} \text {. }
$$


1095 Given that $p_{0}(0)=1$,

$$
\begin{gathered}
\ln p_{0}(\tilde{t})=\frac{-\gamma_{D} D_{0} R_{0}}{\psi_{D}+\psi_{R}}\left(e^{\left(\psi_{D}+\psi_{R}\right) \tilde{t}}-1\right), \\
p_{0}(\tilde{t})=\exp \left\{\frac{-\gamma_{D} D_{0} R_{0}}{\psi_{D}+\psi_{R}}\left(e^{\left(\psi_{D}+\psi_{R}\right) \tilde{t}}-1\right)\right\},
\end{gathered}
$$

which is equation [12].

\section{SI section 8 : Derivation of mutation rate from the Luria-Delbrück experiment} equation:

$$
N_{t}=N_{0} e^{\psi_{N} t}
$$

where $N_{t}$ is the number of wild type cells at time $t$ and $\psi_{N}$ is the per capita growth rate. The wild-type population dynamics are assumed to be deterministic (a reasonable assumption if the initial population size is reasonably large, i.e., $\left.N_{0} \gg 0\right)$. We are also ignoring the loss of wild-type cells to mutational transformation, but this omission is reasonable if the mutation rate is very small relative to per capita wild-type growth rate.

Let the number of mutants be given by a random variable $M_{t}$. This variable takes on non-negative integer values. For a very small interval of time, $\Delta t$, the current number of mutants will either increase by one or remain constant. The probabilities of each possibility are given as follows:

$$
\begin{aligned}
& \operatorname{Pr}\left\{M_{t+\Delta t}=M_{t}+1\right\}=\mu N_{t} \Delta t+\psi_{M} M_{t} \Delta t \\
& \operatorname{Pr}\left\{M_{t+\Delta t}=M_{t}\right\}=1-\left(\mu N_{t}+\psi_{M} M_{t}\right) \Delta t .
\end{aligned}
$$

1113 The two terms on the right-hand side of equation [8.2] give the ways that a mutant can be generated. The first term measures the probability that a wild-type cell undergoes a mutation ( $\mu$ is the mutation rate). The second term gives the probability that a mutant cell divides and produces two mutant cells $\left(\psi_{M}\right.$ is the mutant growth rate). Equation [8.3] is the probability that neither of these processes occur.

Analogous to the procedure in SI section 7 (with $p_{0}(t)=\operatorname{Pr}\left\{M_{t}=0\right\}$ ):

$$
p_{0}(t+\Delta t)=\left(1-\mu N_{t} \Delta t\right) p_{0}(t) \text {. }
$$

By rearranging, taking the limit as $\Delta t \rightarrow 0$, and utilizing equation [8.1], we have 


$$
\frac{d p_{0}(t)}{d t}=-\mu N_{0} e^{\psi_{N} t} p_{0}(t)
$$

This differential equation can be solved in an analogous way as well

$$
\int_{0}^{\tilde{t}} \frac{d p_{0}(t)}{p_{0}(t)}=\int_{0}^{\tilde{t}}-\mu N_{0} e^{\psi_{N} t} d t
$$

$$
\left.\ln p_{0}(t)\right|_{0} ^{\tilde{t}}=\left.\frac{-\mu N_{0}}{\psi_{N}} e^{\psi_{N} t}\right|_{0} ^{\tilde{t}},
$$

$$
\ln p_{0}(\tilde{t})-\ln p_{0}(0)=\frac{-\mu N_{0}}{\psi_{N}} e^{\psi_{N} \tilde{t}}-\frac{-\mu N_{0}}{\psi_{N}} .
$$

Because we assume $M_{0}=0$, we must have $p_{0}(0)=1$, and

$$
\ln p_{0}(\tilde{t})=\frac{-\mu N_{0}}{\psi_{N}}\left(e^{\psi_{N} \tilde{t}}-1\right)
$$

Solving for the mutation rate $\mu$

$$
\mu=-\ln p_{0}(\tilde{t}) \frac{\psi_{N}}{N_{0}\left(e^{\psi_{N} \tilde{t}}-1\right)},
$$

which is equation [14]. This equation can also be expressed as:

$$
\mu=-\ln p_{0}(\tilde{t}) \frac{\psi_{N}}{N_{\tilde{t}}-N_{0}} .
$$

In Luria and Delbrück's original formulation, they measured time in units proportional to the bacterial division cycles. Specifically, a new time variable $z$ is defined:

$$
z=\frac{t}{t_{d} / \ln 2}
$$

where $t_{d}$ is the period required for population doubling during exponential growth.

1138 it is the case that

\section{Because}

$$
N_{0} e^{\psi_{N} t}=N_{0} 2^{t / t_{d}}
$$

$$
\psi_{N}=\frac{1}{t_{d} / \ln 2}
$$

Therefore, $z=\psi_{N} t$ and the equation $N_{t}=N_{0} e^{\psi_{N} t}$ can be expressed as

$$
N_{z}=N_{0} e^{z}
$$

Performing the same analysis on this nondimensionalized equation gives the original formulation (their equations [4] and [5], where our $\mu$ is given by their "a" and our $\tilde{z}$ is given by their "t"):

$$
\mu=\frac{-\ln p_{0}(\tilde{z})}{N_{\tilde{z}}-N_{0}} .
$$




\section{SI section 9 : The LDM MLE derivation}

We start by focusing on $p_{0}(\tilde{t})$, the probability that a population will have no transconjugants at time $\tilde{t}$ (for notational convenience we'll drop the time variable). What we actually measure is the number of independent populations (or wells) that have no transconjugants (call this $w$ ) out of the total number of populations (or wells) tracked (call this $\mathrm{W})$. What is our best estimate of $p_{0}$, given our data $w$ ? That is, what value $p_{0}$ maximizes the likelihood function:

$$
\mathcal{L}\left(p_{0}\right)=\operatorname{Pr}\left\{w \mid p_{0}\right\}=\left(\begin{array}{l}
\mathrm{W} \\
w
\end{array}\right)\left(p_{0}\right)^{w}\left(1-p_{0}\right)^{\mathrm{W}-w}
$$

Because $-\ln (x)$ is a monotonic decreasing function, the value $p_{0}$ that maximizes $\mathcal{L}\left(p_{0}\right)$ will be the same value that minimizes:

$$
\mathrm{L}\left(p_{0}\right)=-\ln \left\{\mathcal{L}\left(p_{0}\right)\right\}
$$

This can be rewritten as follows:

$$
\begin{gathered}
\mathrm{L}\left(p_{0}\right)=-\ln \left\{\left(\begin{array}{l}
\mathrm{W} \\
w
\end{array}\right)\left(p_{0}\right)^{w}\left(1-p_{0}\right)^{\mathrm{W}-w}\right\} \\
\mathrm{L}\left(p_{0}\right)=-\ln \left(\begin{array}{l}
\mathrm{W} \\
w
\end{array}\right)-w \ln p_{0}-(\mathrm{W}-w) \ln \left(1-p_{0}\right)
\end{gathered}
$$

To find the maximum likelihood estimate, we find the critical points of $\mathrm{L}$ by setting its derivative to zero, or:

$$
\frac{d \mathrm{~L}}{d p_{0}}=-\frac{w}{p_{0}}+\frac{\mathrm{W}-w}{1-p_{0}}=0
$$

The maximum likelihood estimate for $p_{0}$ (which we'll denote $\hat{p}_{0}$ ) solves the above equation, or

$$
\begin{gathered}
\frac{\mathrm{W}-w}{1-\hat{p}_{0}}=\frac{w}{\hat{p}_{0}} \\
(\mathrm{~W}-w) \hat{p}_{0}=w\left(1-\hat{p}_{0}\right) \\
\mathrm{W} \hat{p}_{0}-w \hat{p}_{0}=w-w \hat{p}_{0} \\
\mathrm{~W} \hat{p}_{0}=w \\
\hat{p}_{0}=\frac{w}{\mathrm{~W}}
\end{gathered}
$$

This answer makes intuitive sense: the most likely estimate for $p_{0}$ (the probability that a population has no transconjugants) is simply the fraction of the populations that have no transconjugants. 

consider the second derivative:

$$
\frac{d^{2} \mathrm{~L}}{d p_{0}^{2}}=\frac{w}{p_{0}^{2}}+\frac{\mathrm{W}-w}{\left(1-p_{0}\right)^{2}}
$$

Evaluating this derivative at the critical point yields:

$$
\begin{aligned}
& \left.\frac{d^{2} \mathrm{~L}}{d p_{0}^{2}}\right|_{p_{0}=\hat{p}_{0}}=\frac{w}{\left(\frac{w}{\mathrm{~W}}\right)^{2}}+\frac{\mathrm{W}-w}{\left(\frac{\mathrm{W}-w}{\mathrm{~W}}\right)^{2}} \\
& \left.\frac{d^{2} \mathrm{~L}}{d p_{0}^{2}}\right|_{p_{0}=\hat{p}_{0}}=\mathrm{W}^{2}\left(\frac{1}{w}+\frac{1}{\mathrm{~W}-w}\right)
\end{aligned}
$$

As long as $0<w<W$,

which means that $\mathrm{L}$ is convex at $\hat{p}_{0}$ and therefore, this value is a local minimum. In turn, this means that $\hat{p}_{0}$ is a local maximum for $\mathcal{L}$.

\section{SI section 10 : Experimental volume unit conversion using $f$}

In this section, we walk through the addition of $f$ to the LDM estimate. This is

important to maintain the typical units $\frac{\mathrm{ml}}{\mathrm{h} \cdot \mathrm{cfu}}$ used for reporting the conjugation rates. In the "evu" standing for "experimental volume unit" where we will assume there are $f$ evu's per following conversion:

Equivalently,

$$
\breve{D} \frac{\mathrm{cfu}}{\mathrm{evu}}=\frac{\left(D \frac{\mathrm{cfu}}{\mathrm{ml}}\right)}{\left(f \frac{\mathrm{evu}}{\mathrm{ml}}\right)}
$$

$$
\begin{aligned}
\check{R} & =\frac{R}{f} \\
\check{T} & =\frac{T}{f}
\end{aligned}
$$

In our original differential equations, let us multiply both sides of all the differential equations by $1 / f$, yielding: 


$$
\frac{1}{f} \frac{d D}{d t}=\psi \frac{1}{f} D
$$

$$
\frac{1}{f} \frac{d R}{d t}=\psi \frac{1}{f} R-\gamma \frac{1}{f} R(D+T),
$$

$$
\frac{1}{f} \frac{d T}{d t}=\psi \frac{1}{f} T+\gamma \frac{1}{f} R(D+T) .
$$

This can be reworked as

$$
\frac{d \breve{D}}{d t}=\psi \breve{D}
$$

$$
\frac{d \check{R}}{d t}=\psi \check{R}-\gamma \check{R}(D+T),
$$

$$
\frac{d \check{T}}{d t}=\psi \check{T}+\gamma \check{R}(D+T)
$$

We have a mix of densities $\left(\frac{\mathrm{cfu}}{\mathrm{ml}}\right)$ and numbers $\left(\frac{\mathrm{cfu}}{\mathrm{evu}}\right)$ here, so we do the following:

$$
\frac{d \breve{D}}{d t}=\psi \breve{D}
$$

$$
\frac{d \check{R}}{d t}=\psi \check{R}-f \gamma \check{R}\left(\frac{D+T}{f}\right),
$$

$$
\frac{d \check{T}}{d t}=\psi \check{T}+f \gamma \check{R}\left(\frac{D+T}{f}\right) .
$$

Again, making substitutions:

$$
\frac{d \check{D}}{d t}=\psi \breve{D}
$$

If we let

$$
\frac{d \check{R}}{d t}=\psi \check{R}-\{f \gamma\} \check{R}(\breve{D}+\check{T}),
$$$$
\frac{d \check{T}}{d t}=\psi \check{T}+\{f \gamma\} \breve{R}(\breve{D}+\check{T})
$$

$$
\check{\gamma}=f \gamma
$$

we note the units on $\check{\gamma}$ are $\frac{\mathrm{evu}}{\mathrm{h} \cdot \mathrm{cfu}}$. Incorporating this relation, we have

$$
\begin{gathered}
\frac{d \breve{D}}{d t}=\psi \breve{D}, \\
\frac{d \check{R}}{d t}=\psi \check{R}-\check{\gamma} \check{R}(\breve{D}+\check{T}), \\
\frac{d \check{T}}{d t}=\psi \breve{T}+\check{\gamma} \check{R}(\breve{D}+\check{T}) .
\end{gathered}
$$


1220 This set of equations tracks the number of cells (per evu). Thus, if the above equations were used, then the derivations of the LDM estimate could flow exactly like we show in the "Theoretical Framework" section and SI section 7. That is, the following will be correct:

$$
\check{\gamma}=-\ln p_{0}(\tilde{t})\left(\frac{\psi_{D}+\psi_{R}}{\check{D}_{0} \check{R}_{0}\left(e^{\left(\psi_{D}+\psi_{R}\right) \tilde{t}}-1\right)}\right)
$$

Note, no correction is needed on $p_{0}(\tilde{t})$ as everything is in terms of numbers, which was how this quantity was derived. Because $\breve{D}=\frac{D}{f}$ and $\breve{R}=\frac{R}{f}$, we can rewrite the above as

$$
\check{\gamma}=-\ln p_{0}(\tilde{t})\left(\frac{\psi_{D}+\psi_{R}}{\frac{D_{0}}{f} \frac{R_{0}}{f}\left(e^{\left(\psi_{D}+\psi_{R}\right) \tilde{t}}-1\right)}\right)
$$

1227 Or:

$$
\frac{\check{\gamma}}{f}=f\left\{-\ln p_{0}(\tilde{t})\left(\frac{\psi_{D}+\psi_{R}}{D_{0} R_{0}\left(e^{\left(\psi_{D}+\psi_{R}\right) \tilde{t}}-1\right)}\right)\right\}
$$

Because $\gamma=\frac{\check{\gamma}}{f}$, we have

$$
\gamma=f\left\{-\ln p_{0}(\tilde{t})\left(\frac{\psi_{D}+\psi_{R}}{D_{0} R_{0}\left(e^{\left(\psi_{D}+\psi_{R}\right) \tilde{t}}-1\right)}\right)\right\}
$$

Note that if our evu was $1 \mathrm{ml}$, then $f=1$ and we could use our estimate exactly as written. Generally, we have to correct our original metric by multiplying by $f$.

Now just as a check, let's see what happens when we flip the unit of volume for a

where $\check{\gamma}$ has units $\frac{\mathrm{evu}}{\mathrm{h} \cdot \mathrm{cfu}}$. Using our conversion, we can rewrite the right-hand side as different metric, say SIM. Suppose that we use the $\frac{\mathrm{cfu}}{\mathrm{evu}}$ units for cell densities, which means we are calculating follows:

$$
\check{\gamma}=\psi \ln \left(1+\frac{\check{T}_{\tilde{t}}}{\check{R}_{\tilde{t}}} \frac{\breve{N}_{\tilde{t}}}{\breve{D}_{\tilde{t}}}\right) \frac{1}{\left(\widetilde{N}_{\tilde{t}}-\widetilde{N}_{0}\right)}
$$

Canceling and rearranging yields

$$
\check{\gamma}=\psi \ln \left(1+\frac{T_{\tilde{t}} / f}{R_{\tilde{t}} / f} \frac{N_{\tilde{t}} / f}{D_{\tilde{t}} / f}\right) \frac{1}{\left(N_{\tilde{t}}-N_{0}\right) / f}
$$

$1243 \quad$ However, $\gamma=\frac{\check{\gamma}}{f}$, so we have

$$
\frac{\check{\gamma}}{f}=\psi \ln \left(1+\frac{T_{\tilde{t}}}{R_{\tilde{t}}} \frac{N_{\tilde{t}}}{D_{\tilde{t}}}\right) \frac{1}{\left(N_{\tilde{t}}-N_{0}\right)}
$$




$$
\gamma=\psi \ln \left(1+\frac{T_{\tilde{t}}}{R_{\tilde{t}}} \frac{N_{\tilde{t}}}{D_{\tilde{t}}}\right) \frac{1}{\left(N_{\tilde{t}}-N_{0}\right)}
$$

Therefore, if we flip to cell densities in the new unit $\frac{\mathrm{cfu}}{\mathrm{ml}}$, we will automatically get the conjugation rate in the new unit $\frac{\mathrm{ml}}{\mathrm{h} \cdot \mathrm{cfu}}$. The same applies to the TDR and ASM estimates. Therefore, there are no correction factors needed for these metrics as volume units change. Note, in these cases, the numerical value of the transfer rate in the new units is not generally the same as the rate in the old units $(\gamma \neq \check{\gamma}$ when $f \neq 1)$.

\section{SI section 11 : Stochastic simulation framework}

We developed a stochastic simulation framework using the Gillespie algorithm. We extended the base model (equations [1] - [3]) to include plasmid loss due to segregation at rate $\tau$. Thus, transconjugants are transformed into plasmid-free recipients due to plasmid loss via segregation at rate $\tau_{T}$. The donors are transformed into plasmid-free cells due to plasmid loss via segregation at rate $\tau_{D}$. Therefore, the extended model (equations [11.1] - [11.4]) tracks the change in density of a new population type, plasmidfree former donors $(F)$. In total, the extended model describes the change in density of four populations $(D, R, T$, and $F)$ due to various biological parameters: growth rates $\left(\psi_{D}\right.$, $\psi_{R}, \psi_{T}$, and $\left.\psi_{F}\right)$, conjugation rates $\left(\gamma_{D}\right.$ and $\left.\gamma_{T}\right)$ and segregation rates $\left(\tau_{D}\right.$ and $\left.\tau_{T}\right)$. For simplicity, here we assume donor-to-recipient conjugation rate is equal to donor-toplasmid-free-former-donor conjugation rate and represent both with $\gamma_{D}$. In addition, we assume transconjugant-to-recipient conjugation rate is equal to transconjugant-toplasmid-free-donor conjugation rate and represent both with $\gamma_{T}$.

$$
\begin{aligned}
& \frac{d D}{d t}=\psi_{D} D+\left(\gamma_{D} D+\gamma_{T} T\right) F-\tau_{D} D, \\
& \frac{d R}{d t}=\psi_{R} R-\left(\gamma_{D} D+\gamma_{T} T\right) R+\tau_{T} T \\
& \frac{d T}{d t}=\psi_{T} T+\left(\gamma_{D} D+\gamma_{T} T\right) R-\tau_{T} T \\
& \frac{d F}{d t}=\psi_{F} F-\left(\gamma_{D} D+\gamma_{T} T\right) F+\tau_{D} D .
\end{aligned}
$$

Each simulation examined a biological process (i.e., growth, conjugation, or segregation) in isolation by manipulating one or two of the relevant parameters in order 
1268 initiated with a positive density of donors $\left(D_{0}\right)$, a positive density of recipients $\left(R_{0}\right)$, 1269 biological parameters for each population type, and then run for a specified incubation time $\tilde{t}$. For each initial parameter setting, we simulated 10,000 parallel populations and calculated the conjugation rate using each method (TDR, SIM, ASM, and LDM). Each estimate has different requirements for calculating the conjugation rate. The LDM estimate needs multiple populations to calculate $\hat{p}_{0}(\tilde{t})$. Thus, for a single estimate via LDM, we reserved 100 parallel populations, where 99 were used to calculate $\hat{p}_{0}(\tilde{t})$ and the remaining population was used to calculate initial and final cell densities. In other words, the 10,000 parallel populations yielded 100 replicate LDM estimates for each parameter setting. For the other methods, we calculated a conjugation rate for each simulated population. In other words, the 10,000 simulated populations yielded 10,000 replicate estimates for each initial parameter setting.

The window of time for population growth (i.e., incubation time $\tilde{t}$ ) is different for the LDM vs. the other methods. A non-zero conjugation rate can be calculated for TDR, SIM, and ASM whenever there is a non-zero number of transconjugants. This contrasts with the LDM since conjugation rate is calculated when a proportion of populations have zero transconjugants. Given different incubation time requirements for each method, we use a deterministic simulation for each parameter setting to find the incubation time $(\tilde{t})$ where we calculated conjugation rate. For the LDM, the incubation time was chosen to occur where on average a single transconjugant was present in the population. For the other methods, the incubation time occurred where on average ten transconjugant cells were present in the population. For Figure 2, we used a "baseline" set of parameters $\left(\psi_{D}=\right.$ $\left.\psi_{R}=\psi_{T}=\psi_{F}=1, \gamma_{D}=\gamma_{T}=1 \times 10^{-6}, \tau_{D}=\tau_{T}=0\right)$ and initial densities $\left(D_{0}=R_{0}=\right.$ $1 \times 10^{2}$ ) unless otherwise indicated. Given that the Gillespie algorithm is computationally expensive, we chose low initial densities and an unusually high conjugation rate. See below and Figure 3 for more realistic rates.

For Figure 3, the conjugation rate was estimated for each method at 30-minute intervals. The 30-minute time intervals occur over an earlier time interval for the LDM vs. the other methods for reasons described above. For the 30-minute time intervals, we applied estimate-specific filters. For the TDR, SIM, and ASM estimates, the range for these intervals was determined by the condition that at least 90 percent of the simulated populations contained transconjugants at the incubation time $\tilde{t}$. For the LDM estimate, 
the range for these intervals was determined by the condition that at least one parallel population had zero transconjugants. Therefore, estimates for the LDM are shown at earlier incubation times than those for the other methods (Figure 3). Given that Figure 3 explores only three parameter combinations (i.e., one for each panel), the computational expense of the Gillespie algorithm was of less concern than in Figure 2 where there are initial densities $\left(D_{0}=R_{0}=1 \times 10^{5}\right)$ unless otherwise indicated. We note that the Gillespie algorithm is computationally expensive when the densities are very large. Therefore, due to the longer incubation times needed for TDR, SIM, and ASM, only 100 populations of the 10,000 were incubated through the later time intervals (i.e., in Figure 3 , each time point has 100 conjugation rate estimates).

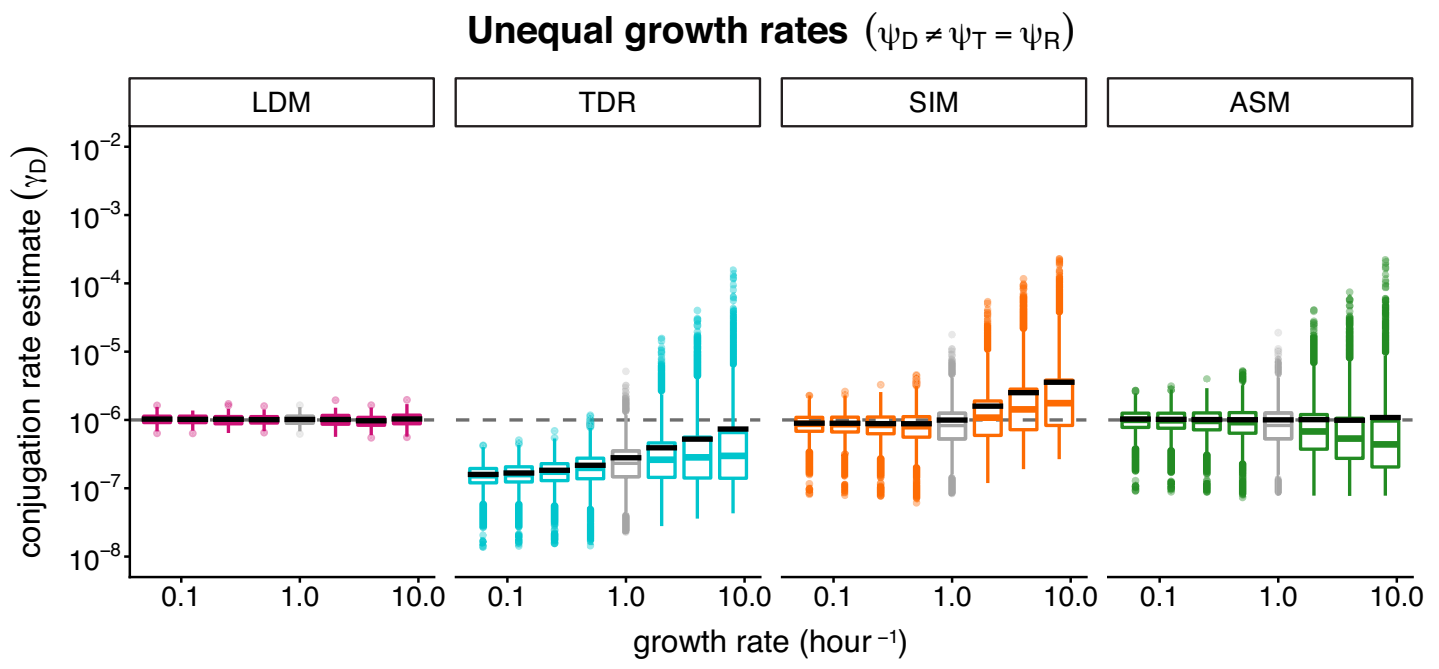

SI Figure 1: The effect of different host growth rates on conjugation rate estimation. The parameter values were $\psi_{D}=\psi_{R}=\psi_{T}=1, \gamma_{D}=\gamma_{T}=1 \times 10^{-6}$, and $\tau_{D}=\tau_{T}=0$ unless otherwise indicated. The dynamic variables were initialized with $D_{0}=R_{0}=1 \times 10^{2}$ and $T_{0}=F_{0}=0$. All incubation times are short but are specific to each parameter setting. Conjugation rate was estimated using 10,000 populations and summarized using boxplots. The gray dashed line indicates the "true" value for the donor conjugation rate. The box contains the $25^{\text {th }}$ to $75^{\text {th }}$ percentile. The vertical lines connected to the box contain 1.5 times the interquartile range above the $75^{\text {th }}$ percentile and below the $25^{\text {th }}$ percentile with the caveat that the whiskers were always constrained to the range of the data. The colored line in the box indicates the median. The solid black line indicates the mean. The boxplots in gray indicate the baseline parameter setting, and all colored plots represent deviation of one or two parameters from baseline. In this plot, we explored unequal host growth rates by tracking simulated populations over a range $\psi_{R}=\psi_{T} \in\{0.0625,0.125,0.24,0.5,1,2,4,8\}$. This captures the situation in which the recipient (and therefore transconjugant) is a different strain or species from the donor and differs in growth rate.

\section{SI section 12 : The LDM protocol}

Here we provide a general protocol for implementing the LDM in the laboratory. 
additions that may be useful if the experimental system allows. For the main body of this section, we left out these "additions" and only included the minimum supplies, strains, and selection conditions needed to estimate conjugation rate using the LDM. For a more general overview of the LDM see the "The LDM Implementation" section.

To start, identify the donor and recipient strains to be used (SI Table 5). The recipients must contain a selectable marker unique to the recipient host strain $\left(S_{R H}\right)$. The donors must also contain a unique selectable marker, but it will be encoded on the donor's conjugative plasmid $\left(S_{P}\right)$. The combination of the unique selectable markers allows for selection of the transconjugant cell type, a recipient strain containing the conjugative plasmid.

SI Table 5: Overview of cell types in the conjugation assay.

\begin{tabular}{|c|l|l|}
\hline Cell type & Cell type description & Selectable marker $(S)$ \\
\hline$R$ & Plasmid-free recipients & Recipient host marker $\left(S_{R H}\right)$ \\
\hline$D$ & Plasmid donors & Donor plasmid marker $\left(S_{P}\right)$ \\
\hline$T$ & Transconjugants & $\begin{array}{l}\text { Recipient host marker }\left(S_{R H}\right) \\
\text { Donor plasmid marker }\left(S_{P}\right)\end{array}$ \\
\hline
\end{tabular}

Part I. Characterize cell types (estimated time $=4$ days)

Four key pieces of information necessary to proceed with Part II are determined here. (1) The approximate cell density for donors and recipients after overnight growth. (2) The minimum inhibitory concentrations (MIC) for the donors, recipients, and transconjugants. If the selectable markers involve antibiotic resistance, this will identify the antibiotic concentration (selection for $S_{R H}+S_{P}$ ) to select for transconjugants and inhibit donors and recipients. (3) Verifying the conjugative plasmid will transfer horizontally between cells in liquid culture. Note that some plasmids transfer inefficiently in liquid culture. (4) The length of lag phase for donor and recipient cells. This information is needed because the donors and recipients are mixed in the exponential phase when determining "the conjugation window" (Part II) and executing the LDM conjugation assay (Part III). 
Step I.1.1 Start three replicate overnight cultures from freezer stocks of the donors and recipients (SI Figure 2a). Include selection for $S_{P}$ in the donor cultures to ensure plasmid maintenance.

Step I.1.2 Incubate cultures.

Step I.2.1 Transfer the donor and recipient cultures created in Step I.1.1 using a 1:100 dilution (SI Figure 2b). Include selection for $S_{P}$ in the donor cultures to ensure plasmid maintenance. cultures created in Step I.1.1 using a 1:100 dilution. Mix the diluted donors and recipients at a 1:1 ratio (SI Figure $2 b$ ).

Note: These mating cultures allow for plasmid conjugation between donors and recipients to create transconjugants. This will (1) verify that transconjugants can form in the liquid condition, (2) generate the

Step I.2.2 To start three replicate mating cultures, dilute the donor and recipient transconjugant strain that will verify the transconjugant-selecting conditions during the MIC assay.

Step I.2.3 Incubate cultures.

Note: If antibiotic selection is used to maintain the donor plasmid, wash donor cells (through three repeated rounds of centrifugation, removal of the supernatant, addition of an equal volume of saline, and vortexing) before

Step I.3.1 Determine the overnight culture density for the donor and recipient cultures created in Step I.2.1, for example by creating a dilution series and separately plating each culture on non-selective medium. experimenter tailor Part II most effectively (see Figure 4a)

Step I.3.2 Determine the minimum inhibitory concentration (MIC) for all cell types (donors, recipients and transconjugants) using transconjugant selection (selection for $S_{R H}+S_{P}$ ).

Note: The MIC needs to be in the growth format planned for the remaining phases (container, temperature, growth medium type, etc.). Since drug 
interaction effects can occur, it is not appropriate to assume that the MIC values established for two strains with a single selectable marker can be combined to yield conditions that will inhibit a strain with both selectable markers (i.e., the MIC for the donor and the recipient are not necessarily concentrations that will inhibit the transconjugant).

I.3.2.1 Create the MIC microtiter plate with a twofold gradient of transconjugant-selecting medium across the rows. See SI Figure 2c, for example.

I.3.2.2 Dilute the donor, recipient and mating cultures created on Day I.2 using a 1:100 dilution. Inoculate the MIC plate with the diluted donors, recipients, and mating cultures in the appropriate columns.

Note: If transconjugants are at too of low of a percentage in the mating cultures, a transconjugant may not get into the MIC microtiter plate. Thus, the MIC for the "transconjugant" may appear to be equal to the maximum MIC of the donors and recipients. In this case, a mating culture should be plated on transconjugant-selecting agar plates to isolate a transconjugant and then determine the MIC.

I.3.2.3 Incubate the MIC microtiter plate.

Step I.3.3 Characterize strain's growth using a spectrophotometer.

I.3.3.1 Dilute the donor and recipient cultures created on Day I.2. using a 1:100 dilution and inoculate three replicate wells for both the diluted donors and recipients.

I.3.3.2 Incubate the microtiter plate in a spectrophotometer using the discontinuous kinetic reading setting which will measure the optical density (O.D.) of each growing culture at frequent intervals.

Note: The spectrophotometer's setting for the kinetic read should include shaking and temperature control which matches the growth conditions used for culturing the strains.

Day 1.4

Step I.4.1 Calculate the overnight culture density by counting the cfu on the agar plates from Step I.3.1. 
Step I.4.2 Determine the transconjugant selection MIC for each cell type by analyzing the MIC microtiter plate created in Step I.3.2.

Note: The transconjugant-selecting concentration to be chosen for Part II should be below the MIC for transconjugants and above the MIC for donors and recipients. All the tested concentrations in SI Figure $2 d$ are usable for Part II. of lag phase for the donor and recipient strains.

Note: In Part II and Part III, the donors and recipient strains need to grow through lag phase and be mixed while in exponential phase. The length of lag phase may differ between donors and recipients.

Step I.4.3 Analyze the growth kinetic readings from Step I.3.3 to determine the length wells for the transconjugant strain isolated in Part I.

Day II.2

Step II.2.1 Transfer cultures by repeating Step I.2.1 and Step I.2.3 from Day I.2 including the transconjugant cultures.

Part II. Determine the conjugation window (estimated time $=4$ days)

Day II.1

Step II.1.1 Start overnight cultures by repeating Day I.1 and adding in three replicate

Day II.3

Step II.3.1 Transfer the donor and recipient cultures created in Step II.2.1 using a 1:100 dilution. Add selection for $S_{P}$ to the donor cultures to ensure plasmid maintenance.

Step II.3.2 Incubate the donor and recipient cultures created in Step II.3.1 until both are in exponential phase. The incubation time for each strain was determined in Step I.4.3.

Step II.3.3 Make a 10-fold dilution series of the donor and recipient cultures after the incubation in Step II.3.2 and plate appropriate dilutions to determine densities. Also, dilute the transconjugant cultures created in Step II.2.1. Note: Reserve a portion of these dilutions to create control wells in a future step. 
Step II.3.4 Mix a portion of the corresponding donor and recipient dilutions created in Step II.3.3.

Note: If antibiotic selection is used to maintain the donor plasmid, wash donor cells as described previously.

Step II.3.5 Add the diluted mixed cultures created in Step II.3.4 into the corresponding columns in a deep-well microtiter plate (Figure 4a gray columns). Create multiple replicate wells for each density-time treatment (see Figure 4a and $4 \mathrm{~b}$ for an example microtiter plate map).

Note: The culture will be diluted 1:10 with transconjugant-selecting medium in a future step. Thus, add a culture volume which allows for a 1:10 dilution in the deep-well. This will vary depending on the volume capacity of the deep-well plate used.

Step II.3.6 Add the diluted donor, recipient, and transconjugant cultures created in Step II.3.3 into the separate corresponding columns (Figure 4a red, blue, and purple column).

Note: Multiple dilutions were created for the donors, recipients, and transconjugants in Step II.3.3. All of the dilutions can be used for controls by using an additional deep-well plate. Figure 4a shows a simple scenario where the densest dilution is used for the control column.

Step II.3.7 Add the diluted cultures for the donors and recipients created in Step II.3.3 into additional separate columns (not shown in Figure 4) to be used as mating controls at each time point.

Note: The mating controls are created at each time point by mixing donors and recipients and immediately adding transconjugant-selecting media. The mating controls confirm that plasmid conjugation is not occurring after the transconjugant-selecting medium is added.

Step II.3.8 At each time point,

II.3.8.1 Mix the donor and recipient cultures created for the mating controls in Step II.3.7 into an empty well in the corresponding row (i.e., the mating control column).

II.3.8.2 Add transconjugant-selecting medium to all wells in the designated row(s) which dilutes the cultures ten-fold (see Figure 4b). 
1465

1466

1467

1468

1469

1470

1471

1472

1473

1474

1475

1476

1477

1478

1479

1480

1481

1482

1483

1484

1485

1486

1487

1488

1489

1490

1491

1492

1493

1494

1495

Step II.3.9 Incubate the microtiter plate until turbidity is stable.

Note: Since transconjugants are rare, it will take longer for cultures to reach stationary phase. Thus, even for fast-growing bacteria this incubation may be longer than one day.

Note: The donor and recipient control wells should stay clear throughout incubation. If turbidity starts to occur, antibiotic may be degrading.

Day II.4

Step II.4.1 Calculate the culture density from Step II.3.1 by counting the cfu on the agar plates created in Step II.3.3.

Step II.4.2 Record the turbidity results from the deep-well plate(s) (Figure 4c).

Step II.4.3 Analyze the data from Step II.4.1 and Step II.4.2 then select a target incubation time $\left(\tilde{t}^{\prime}\right)$ and target initial density for the donors $\left(D_{0}^{\prime}\right)$ and recipients $\left(R_{0}^{\prime}\right)$ to use in Part III (see Figure 4c for an example).

\section{Part III. Executing the actual LDM conjugation assay (estimated time $=4$ days)}

Day III.1

Step III.1.1 Start overnight cultures by repeating Step II.1.1.

Day III.2

Step III.2.1 Transfer cultures by repeating Step II.2.1.

Day III.3

Step III.3.1 Create donor and recipient cultures in exponential phase by repeating Step II.3.1 and Step II.3.2.

Step III.3.2 Dilute the donor and recipient cultures from Step III.3.1 to the target initial densities $\left(D_{0}^{\prime}, R_{0}^{\prime}\right)$. Dilute the transconjugant cultures created in Step III.2.1. Note: Reserve a portion of the dilutions to create control wells.

Step III.3.3 Mix a portion of the diluted donor and recipient cultures created in Step III.3.2.

Step III.3.4 Add the diluted mixed culture created in Step III.3.3 into the corresponding wells in a deep-well microtiter plate (Figure 4d gray wells).

Note: Add additional culture volume to the mating wells to be used to determine the initial and final densities. Thus, after the additional volume is 
removed for the initial density plating $\left(D_{0}, R_{0}\right)$ the remaining culture volume will match the other mating wells during the incubation time.

Step III.3.5 Add the diluted donor, recipient, and transconjugant cultures created in Step III.3.2 into the corresponding separate wells (Figure 4d red, blue, and purple wells).

Step III.3.6 Add the diluted donor and recipient cultures created in Step III.3.2 into 1502 additional wells (not shown in Figure 4) to be used as mating controls. Note: The mating controls are created after the incubation time $(\tilde{t})$ by mixing donor and recipient cultures and immediately adding transconjugantselecting medium. The mating controls confirm that the donors and recipients cannot grow in the transconjugant-selecting medium and that plasmid conjugation is not occurring after transconjugant-selecting medium is added. One deep-well plate can be used for Part III by rearranging the microtiter plate map shown in Figure $4 d$ to include the extra wells needed for the mating controls). (see Figure 4d).

Step III.3.11 Incubate the microtiter plate.

Note: Since transconjugants are rare, it will take longer for cultures to reach stationary phase. Thus, even for fast-growing bacteria this incubation may be longer than one day.

Step III.3.7 Determine initial densities of donor and recipients $\left(D_{0}, R_{0}\right)$ by dilutionplating from three designated mating wells.

Step III.3.8 Incubate the deep well plate for the chosen time $(\tilde{t})$.

Step III.3.9 Determine final densities of donor and recipients $\left(D_{\tilde{t}}, R_{\tilde{t}}\right)$ by dilution-plating from the three designated mating wells used in Step III.3.7.

Day III.4

Step III.4.1 Calculate the initial $\left(D_{0}, R_{0}\right)$ and final densities $\left(D_{\tilde{t}}, R_{\tilde{t}}\right)$ by counting the cfu on the agar plates from Step III.3.7 and Step III.3.9.

Step III.4.2 Record the turbidity results from the deep-well plate (Figure 4d). 
Step III.4.3 Analyze the data from Step III.4.1 and Step III.4.2 to calculate the donor conjugation rate using the LDM estimate (equation [15]).

Protocol "additions" to consider:

- Add an additional selectable marker to either the donor host strain $\left(S_{D H}\right)$ or the recipient host strain $\left(S_{R H 2}\right)$. Since the selectable marker in the recipient host strain $\left(S_{R H}\right)$ is often chromosomally encoded through a single mutation, the donor may easily become resistant to the selecting agent (e.g., corresponding antibiotic) through a similar or different mutation in the donor chromosome. Thus, it becomes valuable to be able to distinguish transconjugant-containing populations from mutant-donor-containing populations through an additional marker. This type of mutant arose in our experiments. However, our donor strain had an additional selectable marker $\left(S_{D H}\right)$, thus the final microtiter plate in the LDM assay was pin replicated into medium selecting for such donors (selection for $S_{D H}+S_{P}+S_{R H}$ ). All mutant-donor-containing populations were identified and eliminated from the LDM calculation. If an additional selectable marker was available for the recipient host strain $\left(S_{R H 2}\right)$, the transconjugant-selecting medium could have all three selective agents (selection for $S_{P}+S_{R H}+S_{R H 2}$ ); here, a spurious result would require two mutations in the same donor lineage, which occurs with a very low probability.

- Adding additional densities and time intervals. In Figure 4, we have shown a version of Part II. This portion of the assay is highly customizable depending on the strains that are used. If the experimenter has an idea of the conjugation window, then Part II could be simplified. If the experimenter is working with strains that have large unknowns, then adding additional densities or time intervals is advisable.

- For a more detailed protocol, see https://github.com/livkosterlitz/LDMprotocol/wiki. 
bioRxiv preprint doi: https://doi.org/101101/2021.01.06 425583; this version posted December 3, 2021. The copyright holder for this preprint (which was not certified by peer review) is the author/funder, who has granted bioRxiv a license to display the preprint in perpetuity. It is made available under aCC-BY-NC-ND 4.0 International license.

a

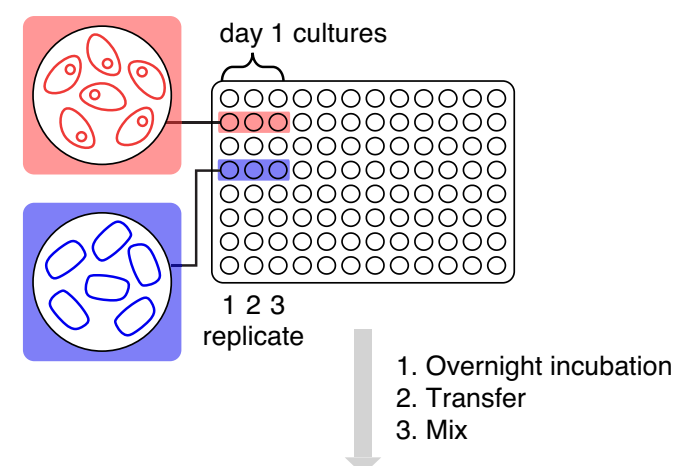

b
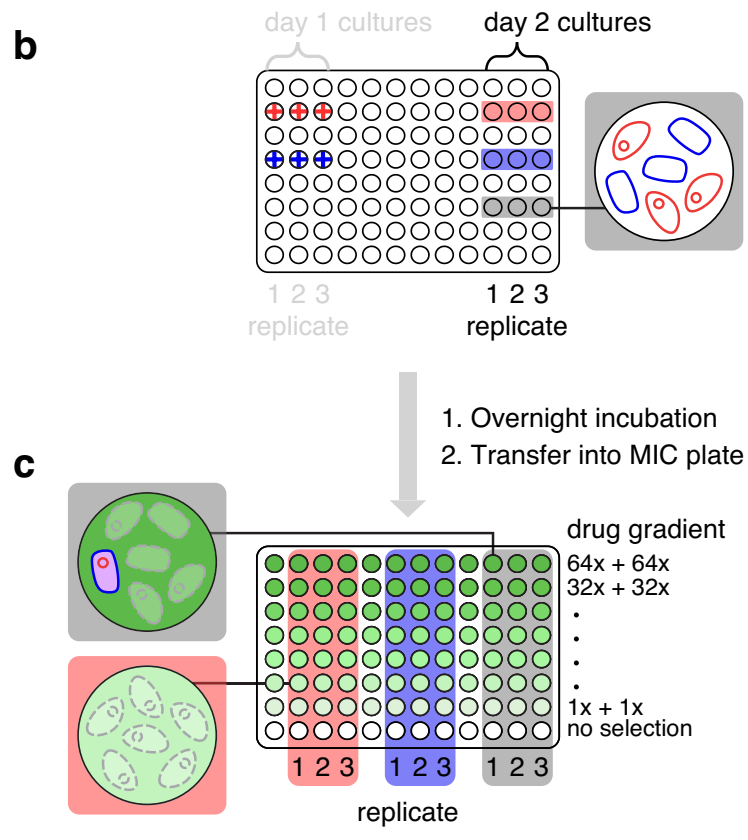

d

Long incubation

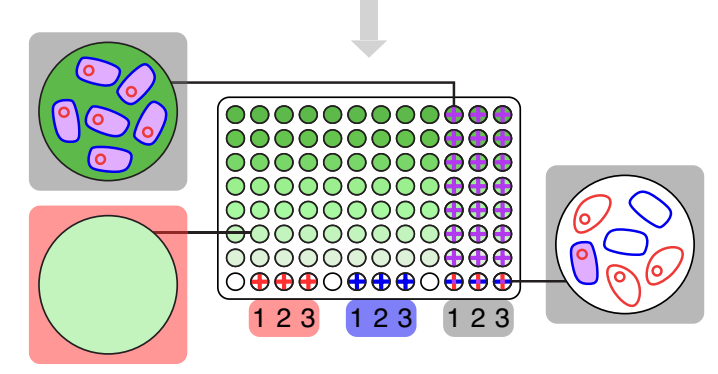

SI Figure 2: Protocol overview for determining the minimum inhibitory concentration in Part I. (a) On day 1, the donors (red) and recipients (blue) are inoculated into three replicate wells in the standard 96-well microtiter plate. (b) On day 2 after incubation of the microtiter plate in a, the grown donor (red crosses) and recipient (blue crosses) replicate cultures are transferred (and diluted) into new wells. In addition, the grown donor a nd recipient are mixed to inoculate 
three replicate mating wells (gray) to allow for plasmid transfer between donors and recipients to create transconjugants. (c) On day 3 after incubation, the drug microtiter plate is created using transconjugant-selecting medium (selection for $S_{R H}+S_{P}$ ) over a two-fold gradient. The bottom row of the microtiter plate contains non-selective medium thus serves as a positive control for bacterial growth. The corresponding grown culture from part (b) is diluted and inoculated into the designated columns in the drug microtiter plate. (d) After incubation of the microtiter plate in (c), $\mathrm{MIC}$ is determined for the donors, recipients, and for transconjugants (purple) that arose in the mating cultures. For recipients (blue cross) and donors (red cross), the MIC for transconjugant selecting medium is below the $1 \mathrm{X}$ drug concentration. For transconjugants (purple cross), the MIC for transconjugant-selecting medium is above the 64X drug concentration. In the bottom row with non-selective medium, all cell types can be present (mixed cross with blue, red and purple).

\section{SI section 13 : Probability generating function and low-order moments}

Keller and Antal (2015) ${ }^{38}$ studied a generalization of the process explored by Luria and

from a single cell as follows:

$$
N_{t}=f(t)=e^{\delta t}
$$

Each wildtype cell generates a mutant cell at a rate $v^{\prime}$, which grows as a stochastic birth process with rate $\alpha$ (Keller and Antal studied a supercritical birth-death process, but we mutants at an arbitrary time:

$$
G(z, t)=\exp \left\{\frac{\nu}{\delta}\left(F\left(1, \kappa ; 1+\kappa ; \frac{z}{z-1} e^{-\alpha t}\right)-e^{\delta t} F\left(1, \kappa ; 1+\kappa ; \frac{z}{z-1}\right)\right)\right\},
$$

where $F$ is the Gaussian hypergeometric function and $\kappa=\frac{\delta}{\alpha}$. an instance of their formulation by making the following substitutions:

$$
\begin{gathered}
\delta=\psi_{D}+\psi_{R}, \\
v=\gamma_{D} D_{0} R_{0}, \\
\alpha=\psi_{T} .
\end{gathered}
$$

With these substitutions, the generating function becomes: 


$$
\begin{aligned}
G(z, t)=\exp & \left\{\frac { \gamma _ { D } D _ { 0 } R _ { 0 } } { \psi _ { D } + \psi _ { R } } \left(F\left(1, \frac{\psi_{D}+\psi_{R}}{\psi_{T}} ; 1+\frac{\psi_{D}+\psi_{R}}{\psi_{T}} ; \frac{z}{z-1} e^{-\psi_{T} t}\right)\right.\right. \\
& \left.\left.-e^{\left(\psi_{D}+\psi_{R}\right) t} F\left(1, \frac{\psi_{D}+\psi_{R}}{\psi_{T}} ; 1+\frac{\psi_{D}+\psi_{R}}{\psi_{T}} ; \frac{z}{z-1}\right)\right)\right\} .
\end{aligned}
$$

Because

$$
G(z, t)=\sum_{n=0}^{\infty} p_{n}(t) z^{n}
$$

$$
\left.F\left(1, \frac{\psi_{D}+\psi_{R}}{\psi_{T}} ; 1+\frac{\psi_{D}+\psi_{R}}{\psi_{T}} ; 0\right)=1\right):
$$

$$
p_{0}(t)=G(0, t)=\exp \left\{\frac{-\gamma_{D} D_{0} R_{0}}{\psi_{D}+\psi_{R}}\left(e^{\left(\psi_{D}+\psi_{R}\right) t}-1\right)\right\},
$$

which agrees with the result from SI section 7. and 9 from Keller and Antal) for the transconjugants:

$$
\begin{gathered}
E\left[T_{t}\right]= \begin{cases}\gamma_{D} D_{0} R_{0} e^{\left(\psi_{D}+\psi_{R}\right) t} t & \text { if } \psi_{D}+\psi_{R}=\psi_{T} D_{0} R_{0}\left(e^{\left(\psi_{D}+\psi_{R}\right) t}-e^{\psi_{T} t}\right) \\
\psi_{D}+\psi_{R}-\psi_{T} & \text { if } \psi_{D}+\psi_{R} \neq \psi_{T}\end{cases} \\
\operatorname{Var}\left[T_{t}\right]=\left\{\begin{array}{lr}
\frac{2 \gamma_{D} D_{0} R_{0}\left(e^{2\left(\psi_{D}+\psi_{R}\right) t}-e^{\left(\psi_{D}+\psi_{R}\right) t}\right)}{\psi_{D}+\psi_{R}}-\gamma_{D} D_{0} R_{0} e^{\left(\psi_{D}+\psi_{R}\right) t} t & \text { if } \psi_{D}+\psi_{R}=\psi_{T} \\
\frac{2 \gamma_{D} D_{0} R_{0}\left(e^{\left(\psi_{D}+\psi_{R}\right) t / 2}-e^{\left(\psi_{D}+\psi_{R}\right) t}\right)}{\psi_{D}+\psi_{R}}+2 \gamma_{D} D_{0} R_{0} e^{\left(\psi_{D}+\psi_{R}\right) t} t & \text { if } \psi_{D}+\psi_{R}=2 \psi_{T} \\
\gamma_{D} D_{0} R_{0}\left\{\frac{2 e^{2 \psi_{T} t}\left(\psi_{T}-\left(\psi_{D}+\psi_{R}\right)\right)-e^{\psi_{T} t}\left(2 \psi_{T}-\left(\psi_{D}+\psi_{R}\right)\right)+\left(\psi_{D}+\psi_{R}\right) e^{\left(\psi_{D}+\psi_{R}\right) t}}{\left(2 \psi_{T}-\left(\psi_{D}+\psi_{R}\right)\right)\left(\psi_{T}-\left(\psi_{D}+\psi_{R}\right)\right)}\right\} \text { otherwise }
\end{array}\right.
\end{gathered}
$$

We provide derivations for these expressions in Box 1. In all cases, the variance grows relative to the mean over time (see Box 2 below for the derivations).

\section{Box 1 : Derivations of the first and second central moments}

In this Box, we derive the equations for the moments using terminology close to what Keller and Antal used (focusing on the case where $\kappa \notin\{1,2\}$ ). From above, $N=e^{\delta t}$ and $N^{-1 / \kappa}=e^{\alpha t}$. Keller and Antal denote the number of mutants (analogous to our transconjugants) as $B$. Also, letting $\xi=\frac{z}{z-1}$ and $\mu=\frac{v}{\alpha}$ and dropping the time argument for notational convenience, the probability generating function (PGF) can be written as: 


$$
G_{B}(z)=\exp \left\{\frac{N \mu}{\kappa}\left[\frac{1}{N} F\left(1, \kappa ; 1+\kappa ; \xi N^{-\frac{1}{\kappa}}\right)-F(1, \kappa ; 1+\kappa ; \xi)\right]\right\}
$$

The expected value for the number of mutants can be obtained from the PGF in the usual way:

$$
E[B]=G_{B}^{\prime}(1-)=\lim _{z \rightarrow 1-} G_{B}(z) \frac{N \mu}{1+\kappa} \frac{1}{(z-1)^{2}} \Sigma,
$$

where

$$
\Sigma=-N^{-1-\frac{1}{\kappa}} F\left(2,1+\kappa ; 2+\kappa ; \xi N^{-\frac{1}{\kappa}}\right)+F(2,1+\kappa ; 2+\kappa ; \xi) .
$$

1609

We use the following identity

$$
F(a, b ; c ; z)=(1-z)^{c-a-b} F(c-a, c-b ; c ; z),
$$

1611

to obtain

$$
\begin{aligned}
F(2,1+\kappa ; 2+\kappa ; \xi) & =(1-\xi)^{-1} F(\kappa, 1,2+\kappa, \xi) \\
& =(1-z) F\left(\kappa, 1,2+\kappa, \frac{z}{z-1}\right)
\end{aligned}
$$

Using a related identity

$$
F(a, b ; c ; z)=(1-z)^{-b} F\left(c-a, b ; c ; \frac{z}{z-1}\right),
$$

we get:

$$
\begin{aligned}
F(2,1+\kappa ; 2+\kappa ; \xi) & =(1-z) F\left(\kappa, 1,2+\kappa, \frac{z}{z-1}\right) \\
& =(1-z)^{2} F(2,1,2+\kappa, z)
\end{aligned}
$$

We will use a similar procedure for the first term in $\Sigma$, obtaining:

$$
\begin{aligned}
F\left(2,1+\kappa ; 2+\kappa ; \xi N^{-1 / \kappa}\right) & =\left(1-\xi N^{-1 / \kappa}\right)^{-1} F\left(\kappa, 1,2+\kappa ; \xi N^{-1 / \kappa}\right) \\
& =\left(1-\xi N^{-1 / \kappa}\right)^{-1} F\left(\kappa, 1,2+\kappa ; \frac{x}{x-1}\right) \\
& =\left(1-\xi N^{-1 / \kappa}\right)^{-1}(1-x) F(2,1,2+\kappa ; x) \\
& =\frac{(z-1)^{2}}{\left(z N^{-1 / \kappa}-z+1\right)^{2}} F(2,1 ; 2+\kappa ; x)
\end{aligned}
$$

where $x=\frac{z N^{-1 / \kappa}}{z N^{-1 / \kappa}-z+1}$.

In sum, we have shown that the first derivative of the PGF can be expressed in the following way:

$$
G_{B}^{\prime}(z)=G_{B}(z) \frac{N \mu}{1+\kappa}\left(-\frac{N^{-1-\frac{1}{\kappa}}}{\left(z N^{-\frac{1}{\kappa}}-z+1\right)^{2}} F(2,1 ; 2+\kappa ; x)+F(2,1,2+\kappa, z)\right) .
$$


Using the fact that $\lim _{z \rightarrow 1-} G_{B}(z)=1$ and the Gauss identity $F(a, b ; c ; 1)=\frac{\Gamma(c) \Gamma(c-a-b)}{\Gamma(c-a) \Gamma(c-b)}$ (which holds if $c>a+b)$ we obtain

$$
E[B]=G_{B}^{\prime}(1-)=\frac{N \mu}{\kappa-1}\left(-N^{-1+1 / \kappa}+1\right) .
$$

1631

1632

To calculate the variance for the number of mutants, we note that

$$
G_{B}^{\prime \prime}(1-)=E\left[B^{2}\right]-E[B]
$$

and thus

$$
\begin{aligned}
\operatorname{Var}[B] & =G_{B}^{\prime \prime}(1-)+E[B]-(E[B])^{2} \\
& =G_{B}^{\prime \prime}(1-)+G_{B}^{\prime}(1-)-\left(G_{B}^{\prime}(1-)\right)^{2}
\end{aligned}
$$

From equation [B1.1] we can express the first derivative of the PGF as:

$$
G_{B}^{\prime}(z)=G_{B}(z) \frac{N \mu}{1+\kappa} \Sigma_{1}
$$

It follows that

$$
\begin{aligned}
\operatorname{Var}[B] & =G_{B}^{\prime \prime}(1-)+G_{B}^{\prime}(1-)-\left(G_{B}^{\prime}(1-)\right)^{2} \\
& =\frac{N \mu}{1+\kappa} \lim _{z \rightarrow 1-}\left(\Sigma_{1}+\Sigma_{1}^{\prime}\right) \\
& =E[B]+\frac{N \mu}{1+\kappa} \lim _{z \rightarrow 1-} \Sigma_{1}^{\prime}
\end{aligned}
$$

To calculate the derivative of $\Sigma_{1}$, we note that

$$
\Sigma_{1}=-\frac{N^{-1-\frac{1}{\kappa}}}{\left(z N^{-\frac{1}{\kappa}}-z+1\right)^{2}} F(2,1 ; 2+\kappa ; x)+F(2,1,2+\kappa, z) .
$$

1644

1645

1646

We can calculate the derivative of the second term:

$$
\frac{\partial F(2,1,2+\kappa, z)}{\partial z}=\frac{2}{2+\kappa} F(3,2 ; 3+\kappa ; z),
$$

and of the first term:

$$
\begin{gathered}
\frac{\partial\left[-\frac{N^{-1-1 / \kappa}}{\left(z N^{-1 / \kappa}-z+1\right)^{2}} F(2,1 ; 2+\kappa ; x)\right]}{\partial z} \\
=-N^{-1-1 / \kappa}\left(-\frac{2\left(N^{-1 / \kappa}-1\right)}{\left(N^{-1 / \kappa} Z-z+1\right)^{3}} F(2,1 ; 2+\kappa ; x)\right. \\
\left.+\frac{2 N^{-1 / \kappa}}{\left(N^{-1 / \kappa} z-z+1\right)^{4}} \frac{1}{2+\kappa} F(3,2 ; 3+\kappa ; x)\right)
\end{gathered}
$$

Taken together, and using Gauss' equality,

$$
\lim _{z \rightarrow 1-} \Sigma_{1}^{\prime}=2\left(N^{-1+1 / \kappa}-N^{-1+2 / \kappa}\right) \frac{\kappa+1}{\kappa-1}+\frac{2}{2+\kappa} \frac{(\kappa+2)(\kappa+1)}{(\kappa-1)(\kappa-2)}\left(1-N^{-1+2 / \kappa}\right)
$$



available under aCC-BY-NC-ND 4.0 International license.

$$
\begin{aligned}
& =2 N^{-1+2 / \kappa} \frac{\kappa+1}{\kappa-1}\left(-1-\frac{1}{\kappa-2}\right)+2 N^{-1+1 / \kappa} \frac{\kappa+1}{\kappa-1}+\frac{2(\kappa+1)}{(\kappa-1)(\kappa-2)} \\
& =2 N^{-1+2 / \kappa} \frac{\kappa+1}{2-\kappa}+2 N^{-1+1 / \kappa} \frac{\kappa+1}{\kappa-1}+\frac{2(\kappa+1)}{(\kappa-1)(\kappa-2)}
\end{aligned}
$$

Coming back to

$$
\operatorname{Var}[B]=E[B]+\frac{N \mu}{1+\kappa} \lim _{z \rightarrow 1-} \Sigma_{1}^{\prime}
$$

we obtain

$$
\operatorname{Var}[B]=N \mu\left[\frac{2}{2-\kappa} N^{-1+\frac{2}{\kappa}}+\frac{1}{\kappa-1} N^{-1+\frac{1}{\kappa}}+\frac{\kappa}{(\kappa-1)(\kappa-2)}\right] .
$$

1658

Making the appropriate substitutions yields our expressions for the mean and variance for our transconjugants.

1660

1661

1662

1663

1664

1665

1666

1667

1668

1669

1670

1671

1672

1673

1674

\section{Box 2 : Behavior of the variance relative to the mean over time} In this Box, we will show that the variance in transconjugant numbers grows relative to the mean over time. To do so, we will need to consider several cases.

Let's consider the ratio of the variance to the mean, which we denote $\rho_{t}=\frac{\operatorname{Var}\left[T_{t}\right]}{E\left[T_{t}\right]}$. We will start by focusing on the case where $\psi_{D}+\psi_{R} \neq \psi_{T}$ and $\psi_{D}+\psi_{R} \neq 2 \psi_{T}$ :

$$
\rho_{t}=\frac{\gamma_{D} D_{0} R_{0}\left\{\frac{2 e^{2 \psi_{T} t}\left(\psi_{T}-\left(\psi_{D}+\psi_{R}\right)\right)-e^{\psi_{T} t}\left(2 \psi_{T}-\left(\psi_{D}+\psi_{R}\right)\right)+\left(\psi_{D}+\psi_{R}\right) e^{\left(\psi_{D}+\psi_{R}\right) t}}{\left(2 \psi_{T}-\left(\psi_{D}+\psi_{R}\right)\right)\left(\psi_{T}-\left(\psi_{D}+\psi_{R}\right)\right)}\right\}}{\frac{\gamma_{D} D_{0} R_{0}\left(e^{\left(\psi_{D}+\psi_{R}\right) t}-e^{\psi_{T} t}\right)}{\psi_{D}+\psi_{R}-\psi_{T}}} .
$$

This can be simplified as follows:

$$
\rho_{t}=\frac{2 e^{2 \psi_{T} t}\left(\psi_{T}-\left(\psi_{D}+\psi_{R}\right)\right)-e^{\psi_{T} t}\left(2 \psi_{T}-\left(\psi_{D}+\psi_{R}\right)\right)+\left(\psi_{D}+\psi_{R}\right) e^{\left(\psi_{D}+\psi_{R}\right) t}}{\left(\psi_{D}+\psi_{R}-2 \psi_{T}\right)\left(e^{\left(\psi_{D}+\psi_{R}\right) t}-e^{\psi_{T} t}\right)} .
$$

Letting $\kappa=\frac{\psi_{D}+\psi_{R}}{\psi_{T}}$,

$$
\begin{aligned}
\rho_{t} & =\frac{2 e^{2 \psi_{T} t}(1-\kappa)-e^{\psi_{T} t}(2-\kappa)+\kappa e^{\left(\psi_{D}+\psi_{R}\right) t}}{(\kappa-2)\left(e^{\left(\psi_{D}+\psi_{R}\right) t}-e^{\psi_{T} t}\right)}, \\
\rho_{t} & =\frac{\kappa e^{\left(\psi_{D}+\psi_{R}\right) t}-2(\kappa-1) e^{2 \psi_{T} t}+(\kappa-2) e^{\psi_{T} t}}{(\kappa-2)\left(e^{\left(\psi_{D}+\psi_{R}\right) t}-e^{\psi_{T} t}\right)} .
\end{aligned}
$$

To determine the behavior of this ratio over time we take the derivative with respect to time: 


$$
=\frac{\left\{\begin{array}{l}
\left(\kappa\left(\psi_{D}+\psi_{R}\right) e^{\left(\psi_{D}+\psi_{R}\right) t}-4 \psi_{T}(\kappa-1) e^{2 \psi_{T} t}+(\kappa-2) \psi_{T} e^{\psi_{T} t}\right)(\kappa-2)\left(e^{\left(\psi_{D}+\psi_{R}\right) t}-e^{\psi_{T} t}\right) \\
-\left(\kappa e^{\left(\psi_{D}+\psi_{R}\right) t}-2(\kappa-1) e^{2 \psi_{T} t}+(\kappa-2) e^{\psi_{T} t}\right)(\kappa-2)\left(\left(\psi_{D}+\psi_{R}\right) e^{\left(\psi_{D}+\psi_{R}\right) t}-\psi_{T} e^{\psi_{T} t}\right)
\end{array}\right\}}{\left((\kappa-2)\left(e^{\left(\psi_{D}+\psi_{R}\right) t}-e^{\psi_{T} t}\right)\right)^{2}}
$$

$$
\frac{d \rho_{t}}{d t}=\frac{\left\{\begin{array}{c}
\left(\kappa\left(\psi_{D}+\psi_{R}\right) e^{\left(\psi_{D}+\psi_{R}\right) t}-4 \psi_{T}(\kappa-1) e^{2 \psi_{T} t}+(\kappa-2) \psi_{T} e^{\psi_{T} t}\right)(\kappa-2)\left(e^{\left(\psi_{D}+\psi_{R}\right) t}\right) \\
-\left(\kappa\left(\psi_{D}+\psi_{R}\right) e^{\left(\psi_{D}+\psi_{R}\right) t}-4 \psi_{T}(\kappa-1) e^{2 \psi_{T} t}+(\kappa-2) \psi_{T} e^{\psi_{T} t}\right)(\kappa-2)\left(e^{\psi_{T} t}\right) \\
-\left(\kappa e^{\left(\psi_{D}+\psi_{R}\right) t}-2(\kappa-1) e^{2 \psi_{T} t}+(\kappa-2) e^{\psi_{T} t}\right)(\kappa-2)\left(\left(\psi_{D}+\psi_{R}\right) e^{\left(\psi_{D}+\psi_{R}\right) t}\right) \\
+\left(\kappa e^{\left(\psi_{D}+\psi_{R}\right) t}-2(\kappa-1) e^{2 \psi_{T} t}+(\kappa-2) e^{\psi_{T} t}\right)(\kappa-2)\left(\psi_{T} e^{\psi_{T} t}\right)
\end{array}\right.}{\left((\kappa-2)\left(e^{\left(\psi_{D}+\psi_{R}\right) t}-e^{\psi_{T} t}\right)\right)^{2}},
$$

$$
\begin{gathered}
\frac{d \rho_{t}}{d t}=\frac{\left\{\begin{array}{c}
\left(-4 \psi_{T}(\kappa-1) e^{2 \psi_{T} t}+(\kappa-2) \psi_{T} e^{\psi_{T} t}\right)(\kappa-2)\left(e^{\left(\psi_{D}+\psi_{R}\right) t}\right) \\
-\left(\kappa\left(\psi_{D}+\psi_{R}\right) e^{\left(\psi_{D}+\psi_{R}\right) t}-4 \psi_{T}(\kappa-1) e^{2 \psi_{T} t}\right)(\kappa-2)\left(e^{\psi_{T} t}\right) \\
-\left(-2(\kappa-1) e^{2 \psi_{T} t}+(\kappa-2) e^{\psi_{T} t}\right)(\kappa-2)\left(\left(\psi_{D}+\psi_{R}\right) e^{\left(\psi_{D}+\psi_{R}\right) t}\right) \\
+\left(\kappa e^{\left(\psi_{D}+\psi_{R}\right) t}-2(\kappa-1) e^{2 \psi_{T} t}\right)(\kappa-2)\left(\psi_{T} e^{\psi_{T} t}\right)
\end{array}\right\}}{\left((\kappa-2)\left(e^{\left(\psi_{D}+\psi_{R}\right) t}-e^{\psi_{T} t}\right)\right)^{2}}, \\
\frac{d \rho_{t}}{d t}=\frac{\left(\begin{array}{c}
\left(\psi_{D}+\psi_{R}-2 \psi_{T}\right) 2(\kappa-1)(\kappa-2) e^{2 \psi_{T} t}\left(e^{\left(\psi_{D}+\psi_{R}\right) t}\right) \\
-\left(\psi_{D}+\psi_{R}-\psi_{T}\right)(\kappa-2)^{2} e^{\psi_{T} t}\left(e^{\left(\psi_{D}+\psi_{R}\right) t}\right) \\
-\left(\psi_{D}+\psi_{R}-\psi_{T}\right) \kappa(\kappa-2) e^{\psi_{T} t}\left(e^{\left(\psi_{D}+\psi_{R}\right) t}\right) \\
+2 \psi_{T}(\kappa-1)(\kappa-2) e^{3 \psi_{T} t}
\end{array}\right\}}{\left((\kappa-2)\left(e^{\left(\psi_{D}+\psi_{R}\right) t}-e^{\psi_{T} t}\right)\right)^{2}}
\end{gathered}
$$

$$
\frac{d \rho_{t}}{d t}=\frac{\left\{\begin{array}{c}
\left(\psi_{D}+\psi_{R}-2 \psi_{T}\right) 2(\kappa-1)(\kappa-2) e^{2 \psi_{T} t}\left(e^{\left(\psi_{D}+\psi_{R}\right) t}\right) \\
-\left(\psi_{D}+\psi_{R}-\psi_{T}\right) 2(\kappa-1)(\kappa-2) e^{\psi_{T} t}\left(e^{\left(\psi_{D}+\psi_{R}\right) t}\right) \\
+2 \psi_{T}(\kappa-1)(\kappa-2) e^{3 \psi_{T} t}
\end{array}\right\}}{\left((\kappa-2)\left(e^{\left(\psi_{D}+\psi_{R}\right) t}-e^{\psi_{T} t}\right)\right)^{2}},
$$

$$
\frac{d \rho_{t}}{d t}=\frac{(\kappa-1)(\kappa-2)\left\{(\kappa-2) e^{\left(\psi_{D}+\psi_{R}+2 \psi_{T}\right) t}-(\kappa-1) e^{\left(\psi_{D}+\psi_{R}+\psi_{T}\right) t}+e^{3 \psi_{T} t}\right\}}{\left(\frac{1}{2 \psi_{T}}\right)\left((\kappa-2)\left(e^{\left(\psi_{D}+\psi_{R}\right) t}-e^{\psi_{T} t}\right)\right)^{2}},
$$

$$
\frac{d \rho_{t}}{d t}=\frac{(\kappa-1)(\kappa-2) e^{\psi_{T} t}\left\{(\kappa-2) e^{\left(\psi_{D}+\psi_{R}+\psi_{T}\right) t}-(\kappa-1) e^{\left(\psi_{D}+\psi_{R}\right) t}+e^{2 \psi_{T} t}\right\}}{\left(\frac{1}{2 \psi_{T}}\right)\left((\kappa-2)\left(e^{\left(\psi_{D}+\psi_{R}\right) t}-e^{\psi_{T} t}\right)\right)^{2}}
$$

$$
\frac{d \rho_{t}}{d t}=\frac{(\kappa-1)(\kappa-2)\left\{(\kappa-2) e^{(\kappa+1) \psi_{T} t}-(\kappa-1) e^{\kappa \psi_{T} t}+e^{2 \psi_{T} t}\right\}}{\left(\frac{e^{-\psi_{T} t}}{2 \psi_{T}}\right)\left((\kappa-2)\left(e^{\left(\psi_{D}+\psi_{R}\right) t}-e^{\psi_{T} t}\right)\right)^{2}}
$$




$$
\operatorname{sign}\left[\frac{d \rho_{t}}{d t}\right]=\operatorname{sign}\left[(\kappa-1)(\kappa-2)\left\{(\kappa-2) e^{(\kappa+1) \psi_{T} t}-(\kappa-1) e^{\kappa \psi_{T} t}+e^{2 \psi_{T} t}\right\}\right]
$$

For $\kappa>2$, which is when the growth rate of the transconjugant is less than the average of the donor and recipient growth rates, we have

$$
\operatorname{sign}\left[\frac{d \rho_{t}}{d t}\right]=\operatorname{sign}\left[(\kappa-2) e^{(\kappa+1) \psi_{T} t}-(\kappa-1) e^{\kappa \psi_{T} t}+e^{2 \psi_{T} t}\right] .
$$

For any $t>0$, for $\kappa=2$

$$
\operatorname{sign}\left[\frac{d \rho_{t}}{d t}\right]=\operatorname{sign}\left[0-e^{2 \psi_{T} t}+e^{2 \psi_{T} t}\right]=0 .
$$

Now letting $g(\kappa)=(\kappa-2) e^{(\kappa+1) \psi_{T} t}-(\kappa-1) e^{\kappa \psi_{T} t}+e^{2 \psi_{T} t}$, we have

$$
\begin{gathered}
g^{\prime}(\kappa)=e^{(\kappa+1) \psi_{T} t}+(\kappa-2) \psi_{T} t e^{(\kappa+1) \psi_{T} t}-e^{\kappa \psi_{T} t}-(\kappa-1) \psi_{T} t e^{\kappa \psi_{T} t}, \\
g^{\prime}(\kappa)=\left\{1+(\kappa-2) \psi_{T} t\right\} e^{(\kappa+1) \psi_{T} t}-\left\{1+(\kappa-1) \psi_{T} t\right\} e^{\kappa \psi_{T} t} .
\end{gathered}
$$

$$
g^{\prime}(\kappa)=h(1)-h(0)
$$

But we see that

$$
\begin{gathered}
h^{\prime}(x)=-\psi_{T} t e^{(\kappa+x) \psi_{T} t}+\left\{1+(\kappa-1-x) \psi_{T} t\right\} \psi_{T} t e^{(\kappa+x) \psi_{T} t}, \\
h^{\prime}(x)=\psi_{T} t e^{(\kappa+x) \psi_{T} t}\left(-1+\left\{1+(\kappa-1-x) \psi_{T} t\right\}\right), \\
h^{\prime}(x)=\psi_{T} t e^{(\kappa+x) \psi_{T} t}(\kappa-1-x) \psi_{T} t .
\end{gathered}
$$

1701

1702

$$
h(1)>h(0)
$$

1705

1706

1707

If $\kappa>2$ and $t>0$, then $h^{\prime}(x)>0$ for $0 \leq x \leq 1$. Since $h(x)$ is a continuous function, we must have

This implies that for $\kappa>2$ and $t>0$,

$$
g^{\prime}(\kappa)>0
$$

Since $g(\kappa)$ is also continuous, and since $g(2)=0$, we now know that for $\kappa>2$ and $t>$ 0 ,

$$
g(\kappa)>0 .
$$

1709

1710

Therefore $\operatorname{sign}\left[\frac{d \rho_{t}}{d t}\right]=1$, or the derivative is positive for $t>0$ and $\kappa>2$, which means the variance grows relative to the mean over time.

For $1<\kappa<2$,

$$
\operatorname{sign}\left[\frac{d \rho_{t}}{d t}\right]=\operatorname{sign}\left[-(\kappa-2) e^{(\kappa+1) \psi_{T} t}+(\kappa-1) e^{\kappa \psi_{T} t}-e^{2 \psi_{T} t}\right] .
$$

Letting $\varphi=-(\kappa-2)$, we know that $0<\varphi<1$, and we can rewrite the above

$$
\operatorname{sign}\left[\frac{d \rho_{t}}{d t}\right]=\operatorname{sign}\left[\varphi e^{(3-\varphi) \psi_{T} t}+(1-\varphi) e^{(3-(1+\varphi)) \psi_{T} t}-e^{2 \psi_{T} t}\right]
$$




$$
\begin{gathered}
\varphi e^{(3-\varphi) \psi_{T} t}+(1-\varphi) e^{(3-(1+\varphi)) \psi_{T} t}-e^{2 \psi_{T} t}>e^{\left(3-\left(\varphi^{2}+(1-\varphi)(1+\varphi)\right)\right) \psi_{T} t}-e^{2 \psi_{T} t}, \\
\varphi e^{(3-\varphi) \psi_{T} t}+(1-\varphi) e^{(3-(1+\varphi)) \psi_{T} t}-e^{2 \psi_{T} t}>e^{\left(3-\left(\varphi^{2}+1-\varphi^{2}\right)\right) \psi_{T} t}-e^{2 \psi_{T} t}, \\
\varphi e^{(3-\varphi) \psi_{T} t}+(1-\varphi) e^{(3-(1+\varphi)) \psi_{T} t}-e^{2 \psi_{T} t}>e^{2 \psi_{T} t}-e^{2 \psi_{T} t}, \\
\varphi e^{(3-\varphi) \psi_{T} t}+(1-\varphi) e^{(3-(1+\varphi)) \psi_{T} t}-e^{2 \psi_{T} t}>0 .
\end{gathered}
$$

Therefore $\operatorname{sign}\left[\frac{d \rho_{t}}{d t}\right]=1$, or the derivative is positive for $t>0$ and $1<\kappa<2$, which means the variance grows relative to the mean over time.

Next, we turn to $0<\kappa<1$, where

$$
\operatorname{sign}\left[\frac{d \rho_{t}}{d t}\right]=\operatorname{sign}\left[(\kappa-2) e^{(\kappa+1) \psi_{T} t}-(\kappa-1) e^{\kappa \psi_{T} t}+e^{2 \psi_{T} t}\right] .
$$

For any $t>0$, for $\kappa=1$

$$
\operatorname{sign}\left[\frac{d \rho_{t}}{d t}\right]=\operatorname{sign}\left[-e^{2 \psi_{T} t}-0+e^{2 \psi_{T} t}\right]=0 .
$$

Now letting $g(\kappa)=(\kappa-2) e^{(\kappa+1) \psi_{T} t}-(\kappa-1) e^{\kappa \psi_{T} t}+e^{2 \psi_{T} t}$, we have from above

$$
g^{\prime}(\kappa)=\left\{1+(\kappa-2) \psi_{T} t\right\} e^{(\kappa+1) \psi_{T} t}-\left\{1+(\kappa-1) \psi_{T} t\right\} e^{\kappa \psi_{T} t} .
$$

Letting $h(x)=\left\{1+(\kappa-1-x) \psi_{T} t\right\} e^{(\kappa+x) \psi_{T} t}$, we see

$$
g^{\prime}(\kappa)=h(1)-h(0)
$$

But we see from above that

$$
h^{\prime}(x)=\psi_{T} t e^{(\kappa+x) \psi_{T} t}(\kappa-1-x) \psi_{T} t
$$

If $0<\kappa<1$ and $t>0$, then $h^{\prime}(x)<0$ for $0 \leq x \leq 1$. Since $h(x)$ is a continuous function, we must have

$$
h(1)<h(0)
$$

This implies that for $0<\kappa<1$ and $t>0$,

$$
g^{\prime}(\kappa)<0
$$

Since $g(\kappa)$ is continuous, and since $g(1)=0$, we now know that for $0<\kappa<1$ and $t>$ $0, g(\kappa)>0$. Therefore $\operatorname{sign}\left[\frac{d \rho_{t}}{d t}\right]=1$, or the derivative is positive for $t>0$ and $0<\kappa<1$, which means the variance grows relative to the mean over time.

Now consider the special case of $\kappa=2$ 

available under aCC-BY-NC-ND 4.0 International license.

$$
\begin{gathered}
\rho_{t}=\frac{\frac{2 \gamma_{D} D_{0} R_{0}\left(e^{\psi_{T} t}-e^{2 \psi_{T} t}\right)}{2 \psi_{T}}+2 \gamma_{D} D_{0} R_{0} e^{2 \psi_{T} t} t}{\frac{\gamma_{D} D_{0} R_{0}\left(e^{2 \psi_{T} t}-e^{\psi_{T} t}\right)}{2 \psi_{T}-\psi_{T}}}, \\
\rho_{t}=\frac{\left(e^{\psi_{T} t}-e^{2 \psi_{T} t}\right)+2 \psi_{T} t e^{2 \psi_{T} t}}{\left(e^{2 \psi_{T} t}-e^{\psi_{T} t}\right)} \\
\rho_{t}=\frac{\left(2 \psi_{T} t-1\right) e^{2 \psi_{T} t}+e^{\psi_{T} t}}{\left(e^{2 \psi_{T} t}-e^{\psi_{T} t}\right)}
\end{gathered}
$$

Taking the derivative with respect to time yields

$$
\frac{d \rho_{t}}{d t}=\frac{\left\{\begin{array}{c}
\left(2 \psi_{T} e^{2 \psi_{T} t}+2 \psi_{T}\left(2 \psi_{T} t-1\right) e^{2 \psi_{T} t}+\psi_{T} e^{\psi_{T} t}\right)\left(e^{2 \psi_{T} t}-e^{\psi_{T} t}\right) \\
-\left(\left(2 \psi_{T} t-1\right) e^{2 \psi_{T} t}+e^{\psi_{T} t}\right)\left(2 \psi_{T} e^{2 \psi_{T} t}-\psi_{T} e^{\psi_{T} t}\right)
\end{array}\right\}}{\left(e^{2 \psi_{T} t}-e^{\psi_{T} t}\right)^{2}},
$$

$\frac{d \rho_{t}}{d t}=\psi_{T} \frac{\left(4 \psi_{T} t e^{2 \psi_{T} t}+e^{\psi_{T} t}\right)\left(e^{2 \psi_{T} t}-e^{\psi_{T} t}\right)-\left(\left(2 \psi_{T} t-1\right) e^{2 \psi_{T} t}+e^{\psi_{T} t}\right)\left(2 e^{2 \psi_{T} t}-e^{\psi_{T} t}\right)}{\left(e^{2 \psi_{T} t}-e^{\psi_{T} t}\right)^{2}}$

$$
\frac{d \rho_{t}}{d t}=\psi_{T} \frac{\left\{\begin{array}{c}
4 \psi_{T} t e^{4 \psi_{T} t}+e^{3 \psi_{T} t}-4 \psi_{T} t e^{3 \psi_{T} t}-e^{2 \psi_{T} t} \\
-2\left(2 \psi_{T} t-1\right) e^{4 \psi_{T} t}-2 e^{3 \psi_{T} t}+\left(2 \psi_{T} t-1\right) e^{3 \psi_{T} t}+e^{2 \psi_{T} t}
\end{array}\right\}}{\left(e^{2 \psi_{T} t}-e^{\psi_{T} t}\right)^{2}}
$$

$$
\frac{d \rho_{t}}{d t}=\psi_{T} \frac{\left\{4 \psi_{T} t-2\left(2 \psi_{T} t-1\right)\right\} e^{4 \psi_{T} t}-\left\{4 \psi_{T} t+1-\left(2 \psi_{T} t-1\right)\right\} e^{3 \psi_{T} t}}{\left(e^{2 \psi_{T} t}-e^{\psi_{T} t}\right)^{2}},
$$

$$
\frac{d \rho_{t}}{d t}=\psi_{T} \frac{2 e^{4 \psi_{T} t}-\left\{2 \psi_{T} t+2\right\} e^{3 \psi_{T} t}}{\left(e^{2 \psi_{T} t}-e^{\psi_{T} t}\right)^{2}}
$$

$$
\frac{d \rho_{t}}{d t}=2 \psi_{T} e^{3 \psi_{T} t} \frac{e^{\psi_{T} t}-\psi_{T} t-1}{\left(e^{2 \psi_{T} t}-e^{\psi_{T} t}\right)^{2}}
$$

Using the Taylor expansion

$$
\begin{gathered}
\frac{d \rho_{t}}{d t}=2 \psi_{T} e^{3 \psi_{T} t} \frac{\left(1+\psi_{T} t+\frac{\left(\psi_{T} t\right)^{2}}{2 !}+\frac{\left(\psi_{T} t\right)^{3}}{3 !}+\frac{\left(\psi_{T} t\right)^{4}}{4 !} \ldots\right)-\psi_{T} t-1}{\left(e^{2 \psi_{T} t}-e^{\psi_{T} t}\right)^{2}}, \\
\frac{d \rho_{t}}{d t}=2 \psi_{T} e^{3 \psi_{T} t} \frac{\left(\frac{\left(\psi_{T} t\right)^{2}}{2 !}+\frac{\left(\psi_{T} t\right)^{3}}{3 !}+\frac{\left(\psi_{T} t\right)^{4}}{4 !} \ldots\right)}{\left(e^{2 \psi_{T} t}-e^{\psi_{T} t}\right)^{2}}
\end{gathered}
$$

1756

1757

1758

1759

Therefore, $\frac{d \rho_{t}}{d t}>0$ for $t>0$ and $\kappa=2$, which means the variance grows relative to the mean over time.

Finally, consider the special case of $\kappa=1$

$$
\rho_{t}=\frac{\frac{2 \gamma_{D} D_{0} R_{0}\left(e^{2 \psi_{T} t}-e^{\psi_{T} t}\right)}{\psi_{T}}-\gamma_{D} D_{0} R_{0} e^{\psi_{T} t} t}{\gamma_{D} D_{0} R_{0} e^{\psi_{T} t} t}
$$




$$
\begin{gathered}
\rho_{t}=\frac{2 e^{2 \psi_{T} t}-\left(2+\psi_{T} t\right) e^{\psi_{T} t}}{\psi_{T} t e^{\psi_{T} t}} \\
\rho_{t}=\frac{2 e^{\psi_{T} t}-\left(2+\psi_{T} t\right)}{\psi_{T} t}
\end{gathered}
$$

Using the Taylor expansion

$$
\begin{gathered}
\rho_{t}=\frac{2\left(1+\psi_{T} t+\frac{\left(\psi_{T} t\right)^{2}}{2 !}+\frac{\left(\psi_{T} t\right)^{3}}{3 !}+\frac{\left(\psi_{T} t\right)^{4}}{4 !} \ldots\right)-\left(2+\psi_{T} t\right)}{\psi_{T} t} \\
\rho_{t}=\frac{\psi_{T} t+2\left(\frac{\left(\psi_{T} t\right)^{2}}{2 !}+\frac{\left(\psi_{T} t\right)^{3}}{3 !}+\frac{\left(\psi_{T} t\right)^{4}}{4 !} \ldots\right)}{\psi_{T} t} \\
\rho_{t}=1+2\left(\frac{\psi_{T} t}{2 !}+\frac{\left(\psi_{T} t\right)^{2}}{3 !}+\frac{\left(\psi_{T} t\right)^{3}}{4 !} \ldots\right)
\end{gathered}
$$

Once again, the variance grows relative to the mean over time.

Overall, we have shown that for all $\kappa>0$ and $t>0$, the ratio of the transconjugant variance to the mean number of transconjugants is amplified over time.

\section{SI section 14 : Variance in Estimates}

Here we will focus on two estimates, ASM and LDM, and ask about their variance (enabling us to compare precision). We will focus exclusively on the contributions to this variance coming from the stochasticity in the transconjugant numbers (i.e., ignoring

We start with the ASM estimate (here we express the estimate in terms of growth rate parameters):

$$
\gamma_{D}=\frac{\psi_{D}+\psi_{R}-\psi_{T}}{D_{0} R_{0}\left(e^{\left(\psi_{D}+\psi_{R}\right) \tilde{t}}-e^{\psi_{T} \tilde{t}}\right)} T_{\tilde{t}} .
$$

$$
c_{1}=\frac{\psi_{D}+\psi_{R}-\psi_{T}}{D_{0} R_{0}\left(e^{\left(\psi_{D}+\psi_{R}\right) \tilde{t}}-e^{\psi_{T} \tilde{t}}\right)} .
$$


The variance of the ASM estimate is then

$$
\operatorname{var}\left(\Gamma_{\mathrm{ASM}}\right)=c_{1}{ }^{2}\left\{\operatorname{var}\left(T_{\tilde{t}}\right)\right\}
$$

But we have a closed form expression for $\operatorname{var}\left(T_{\tilde{t}}\right)$. If $\psi_{T} \notin\left\{\psi_{D}+\psi_{R},\left(\psi_{D}+\psi_{R}\right) / 2\right\}$, we 1790 have:

$$
\begin{aligned}
& \operatorname{var}\left(\Gamma_{\mathrm{ASM}}\right)=\left(\frac{\psi_{D}+\psi_{R}-\psi_{T}}{D_{0} R_{0}\left(e^{\left(\psi_{D}+\psi_{R}\right) \tilde{t}}-e^{\psi_{T} \tilde{t}}\right)}\right)^{2} \gamma_{D} D_{0} R_{0}\left\{\frac{\left(\psi_{D}+\psi_{R}\right) e^{\left(\psi_{D}+\psi_{R}\right) \tilde{t}}}{\left(\psi_{D}+\psi_{R}-\psi_{T}\right)\left(\psi_{D}+\psi_{R}-2 \psi_{T}\right)}+\frac{e^{\psi_{T} \tilde{t}}}{\psi_{D}+\psi_{R}-\psi_{T}}-\frac{2 e^{2 \psi_{T} \tilde{t}}}{\psi_{D}+\psi_{R}-2 \psi_{T}}\right\}, \\
& \operatorname{var}\left(\Gamma_{\mathrm{ASM}}\right)=\frac{\gamma_{D}}{D_{0} R_{0}}\left(\frac{\psi_{D}+\psi_{R}-\psi_{T}}{e^{\left(\psi_{D}+\psi_{R}\right) \tilde{t}}-e^{\psi_{T} \tilde{t}}}\right)^{2}\left\{\frac{\left(\psi_{D}+\psi_{R}\right) e^{\left(\psi_{D}+\psi_{R}\right) \tilde{t}}+\left(\psi_{D}+\psi_{R}-2 \psi_{T}\right) e^{\psi_{T} \tilde{t}}-\left(\psi_{D}+\psi_{R}-\psi_{T}\right) 2 e^{2} \psi_{T} \tilde{t}}{\left(\psi_{D}+\psi_{R}-\psi_{T}\right)\left(\psi_{D}+\psi_{R}-2 \psi_{T}\right)}\right\}, \\
& \operatorname{var}\left(\Gamma_{\mathrm{ASM}}\right)=\frac{\gamma_{D}\left(\psi_{D}+\psi_{R}-\psi_{T}\right)}{D_{0} R_{0}}\left\{\frac{\left(\psi_{D}+\psi_{R}\right) e^{\left(\psi_{D}+\psi_{R}\right) \tilde{t}}+\left(\psi_{D}+\psi_{R}-2 \psi_{T}\right) e^{\psi_{T} \tilde{t}}-\left(\psi_{D}+\psi_{R}-\psi_{T}\right) 2 e^{2 \psi_{T} \tilde{t}}}{\left(\psi_{D}+\psi_{R}-2 \psi_{T}\right)\left(e^{\left(\psi_{D}+\psi_{R}\right) \tilde{t}_{-}}-e_{T} \tilde{t}\right)^{2}}\right\} .
\end{aligned}
$$

The formulas for $\psi_{T}=\psi_{D}+\psi_{R}$ and $2 \psi_{T}=\psi_{D}+\psi_{R}$ could also be derived via simple substitution (note, $\lim _{\psi_{T} \rightarrow \psi_{D}+\psi_{R}} c_{1}=1 /\left(D_{0} R_{0} t e^{\left(\psi_{D}+\psi_{R}\right) \tilde{t}}\right)$. These formulas allow us to project variance in the ASM estimate over time due to transconjugant variation if all parameters are known.

We now turn to the LDM estimate:

$$
\gamma_{D}=-\ln \hat{p}_{0}(\tilde{t})\left(\frac{\psi_{D}+\psi_{R}}{D_{0} R_{0}\left(e^{\left(\psi_{D}+\psi_{R}\right) \tilde{t}}-1\right)}\right) .
$$

What we actually measure is the number of populations (or wells) that have no transconjugants (call this $w$ ) out of the total number of populations (or wells) tracked (call this $W)$. As we show in SI section 9, the maximum likelihood estimate of $p_{0}(\tilde{t})$ is

$$
\hat{p}_{0}(\tilde{t})=\frac{w}{\mathrm{~W}}
$$

Of course, from experiment to experiment, there will be variance in the number of populations with no transconjugants. Let us consider a random variable $F$, which represents the fraction of total populations that have no transconjugants. The expectation of $F$ is (we drop the time argument for notational convenience):

$$
\mathrm{E}[F]=\sum_{w=0}^{\mathrm{W}}\left(\begin{array}{l}
\mathrm{W} \\
w
\end{array}\right)\left(p_{0}\right)^{w}\left(1-p_{0}\right)^{\mathrm{W}-w}\left(\frac{w}{\mathrm{~W}}\right)
$$$$
\mathrm{E}[F]=\frac{1}{\mathrm{~W}} \sum_{w=1}^{\mathrm{W}} \frac{\mathrm{W} !}{w !(\mathrm{W}-w) !}\left(p_{0}\right)^{w}\left(1-p_{0}\right)^{\mathrm{W}-w} w,
$$

$$
\mathrm{E}[F]=\frac{1}{\mathrm{~W}} \sum_{w=1}^{\mathrm{W}} \frac{\mathrm{W} !}{(\mathrm{W}-1) !(\mathrm{W}-w) !}\left(p_{0}\right)^{w}\left(1-p_{0}\right)^{\mathrm{W}-w},
$$




$$
\mathrm{E}[F]=p_{0} \sum_{w=1}^{\mathrm{W}} \frac{(\mathrm{W}-1) !}{(\mathrm{w}-1) !((\mathrm{W}-1)-(\mathrm{w}-1)) !}\left(p_{0}\right)^{w-1}\left(1-p_{0}\right)^{(\mathrm{W}-1)-(w-1)}
$$

Letting $i=w-1$, we have

$$
\mathrm{E}[F]=p_{0} \sum_{i=0}^{\mathrm{W}-1} \frac{(\mathrm{W}-1) !}{i !((\mathrm{W}-1)-i) !}\left(p_{0}\right)^{i}\left(1-p_{0}\right)^{(\mathrm{W}-1)-i}
$$

$$
\mathrm{E}[F]=p_{0}
$$

which makes sense. The second central moment of $F$ is

$$
\begin{aligned}
& \operatorname{var}[F]=\sum_{w=0}^{\mathrm{W}}\left(\begin{array}{l}
\mathrm{W} \\
w
\end{array}\right)\left(p_{0}\right)^{w}\left(1-p_{0}\right)^{\mathrm{W}-w}\left(\frac{w}{\mathrm{~W}}-p_{0}\right)^{2} \\
& \operatorname{var}[F]=\sum_{w=0}^{\mathrm{W}}\left(\begin{array}{l}
\mathrm{W} \\
w
\end{array}\right)\left(p_{0}\right)^{w}\left(1-p_{0}\right)^{\mathrm{W}-w}\left(\left(\frac{w}{\mathrm{~W}}\right)^{2}-2 p_{0}\left(\frac{w}{\mathrm{~W}}\right)+\left(p_{0}\right)^{2}\right) \\
& \operatorname{var}[F]=\sum_{w=0}^{\mathrm{W}}\left(\begin{array}{l}
\mathrm{W} \\
w
\end{array}\right)\left(p_{0}\right)^{w}\left(1-p_{0}\right)^{\mathrm{W}-w}\left(\frac{w}{\mathrm{~W}}\right)^{2}-2 p_{0} \sum_{w=0}^{\mathrm{W}}\left(\begin{array}{l}
\mathrm{W} \\
w
\end{array}\right)\left(p_{0}\right)^{w}\left(1-p_{0}\right)^{\mathrm{W}-w}\left(\frac{w}{\mathrm{~W}}\right) \\
& +\left(p_{0}\right)^{2} \sum_{w=0}^{\mathrm{W}}\left(\begin{array}{l}
\mathrm{W} \\
w
\end{array}\right)\left(p_{0}\right)^{w}\left(1-p_{0}\right)^{\mathrm{W}-w} \\
& \operatorname{var}[F]=\left\{\left(\frac{1}{\mathrm{~W}^{2}}\right) \sum_{w=0}^{\mathrm{W}}\left(\begin{array}{l}
\mathrm{W} \\
w
\end{array}\right)\left(p_{0}\right)^{w}\left(1-p_{0}\right)^{\mathrm{W}-w^{2}} w^{2}\right\}-2 p_{0} \mathrm{E}[F]+\left(p_{0}\right)^{2} \\
& \operatorname{var}[F]=\left\{\left(\frac{1}{\mathrm{~W}^{2}}\right) \sum_{w=0}^{\mathrm{W}}\left(\begin{array}{l}
\mathrm{W} \\
w
\end{array}\right)\left(p_{0}\right)^{w}\left(1-p_{0}\right)^{\mathrm{W}-w} w(w-1+1)\right\}-2\left(p_{0}\right)^{2}+\left(p_{0}\right)^{2} \\
& \operatorname{var}[F]=\left\{\left(\frac{1}{\mathrm{~W}^{2}}\right) \sum_{w=2}^{\mathrm{W}} \frac{\mathrm{W} !}{w !(\mathrm{W}-w) !}\left(p_{0}\right)^{w}\left(1-p_{0}\right)^{\mathrm{W}-w} w(w-1)\right. \\
& \left.+\left(\frac{1}{\mathrm{~W}^{2}}\right) \sum_{w=0}^{\mathrm{W}}\left(\begin{array}{l}
\mathrm{W} \\
w
\end{array}\right)\left(p_{0}\right)^{w}\left(1-p_{0}\right)^{\mathrm{W}-w} w\right\}-\left(p_{0}\right)^{2} \\
& \operatorname{var}[F]=\left\{\left(\frac{1}{\mathrm{~W}^{2}}\right) \sum_{w=2}^{\mathrm{W}} \frac{\mathrm{W} !}{(w-2) !(\mathrm{W}-w) !}\left(p_{0}\right)^{w}\left(1-p_{0}\right)^{\mathrm{W}-w}\right. \\
& \left.+\left(\frac{1}{\mathrm{~W}}\right) \sum_{w=0}^{\mathrm{W}}\left(\begin{array}{l}
\mathrm{W} \\
w
\end{array}\right)\left(p_{0}\right)^{w}\left(1-p_{0}\right)^{\mathrm{W}-w}\left(\frac{w}{\mathrm{~W}}\right)\right\}-\left(p_{0}\right)^{2}
\end{aligned}
$$




$$
\begin{gathered}
\operatorname{var}[F]=\left(\frac{\mathrm{W}(\mathrm{W}-1)\left(p_{0}\right)^{2}}{\mathrm{~W}^{2}}\right)\left\{\sum_{w=2}^{\mathrm{W}} \frac{(\mathrm{W}-2) !}{(w-2) !((\mathrm{W}-2)-(w-2)) !}\left(p_{0}\right)^{w-2}(1\right. \\
\left.\left.-p_{0}\right)^{(\mathrm{W}-2)-(w-2)}\right\}+\frac{p_{0}}{\mathrm{~W}}-\left(p_{0}\right)^{2}
\end{gathered}
$$

Letting $j=w-2$, we have

$$
\operatorname{var}[F]=\left(\frac{(\mathrm{W}-1)\left(p_{0}\right)^{2}}{\mathrm{~W}}\right)\left\{\sum_{j=0}^{\mathrm{W}-2}\left(\begin{array}{c}
\mathrm{W}-2 \\
j
\end{array}\right)\left(p_{0}\right)^{j}\left(1-p_{0}\right)^{(\mathrm{W}-2)-j}\right\}+\frac{p_{0}}{\mathrm{~W}}-\left(p_{0}\right)^{2}
$$

$$
\begin{aligned}
& \operatorname{var}[F]=\left(\frac{(\mathrm{W}-1)\left(p_{0}\right)^{2}}{\mathrm{~W}}\right)+\frac{p_{0}}{\mathrm{~W}}-\left(p_{0}\right)^{2}, \\
& \operatorname{var}[F]=\frac{(\mathrm{W}-1)\left(p_{0}\right)^{2}+p_{0}-\mathrm{W}\left(p_{0}\right)^{2}}{\mathrm{~W}},
\end{aligned}
$$

$$
\begin{aligned}
& \operatorname{var}[F]=\frac{-\left(p_{0}\right)^{2}+p_{0}}{\mathrm{~W}}, \\
& \operatorname{var}[F]=\frac{p_{0}\left(1-p_{0}\right)}{\mathrm{W}} .
\end{aligned}
$$

1836

Because we are only focusing on the contribution of the transconjugant variation, all 1838 parameters (initial densities and growth rates will be taken to be fixed). Thus, we can think about the LDM estimate as a random variable $\Gamma_{\mathrm{LDM}}$,

$$
\Gamma_{\mathrm{LDM}}=c_{2} \ln F \text {, }
$$

$$
c_{2}=-\left(\frac{\psi_{D}+\psi_{R}}{D_{0} R_{0}\left(e^{\left(\psi_{D}+\psi_{R}\right) \tilde{t}}-1\right)}\right)
$$

where the constant $c_{2}$ is

The variance of the LDM estimate is then

$$
\operatorname{var}\left(\Gamma_{\mathrm{LDM}}\right)=c_{2}^{2}\{\operatorname{var}(\ln F)\} \text {. }
$$

Here we use a first-order Taylor series approximation for $\ln F$ centered at $\mathrm{E}[F]$ in the following way:

$$
\begin{gathered}
\ln F \approx \ln (\mathrm{E}[F])+\frac{1}{\mathrm{E}[F]}(F-\mathrm{E}[F]), \\
\ln F \approx \frac{F}{\mathrm{E}[F]}+\ln (\mathrm{E}[F])-1 .
\end{gathered}
$$


So, because $\ln (E[F])-1$ is constant, we have

$$
\begin{gathered}
\operatorname{var}[\ln F] \approx \operatorname{var}\left\{\frac{F}{\mathrm{E}[F]}\right\}, \\
\operatorname{var}[\ln F] \approx\left(\frac{1}{\mathrm{E}[F]}\right)^{2} \operatorname{var}[F], \\
\operatorname{var}[\ln F] \approx\left(\frac{1}{p_{0}}\right)^{2}\left(\frac{p_{0}\left(1-p_{0}\right)}{\mathrm{W}}\right), \\
\operatorname{var}[\ln F] \approx \frac{1}{\mathrm{~W}}\left(\frac{1}{p_{0}}-1\right) .
\end{gathered}
$$

This approximation will be accurate when the deviation between $F$ and $\mathrm{E}[F]$ is very small (i.e., $\left.\frac{|F-\mathrm{E}[F]|}{\mathrm{E}[F]} \ll 1\right)$. As $\mathrm{W}$ (the number of replicate populations in the experiment) gets large,

Now, we have the following expression for $p_{0}$ (reintroducing the time argument):

$$
p_{0}(\tilde{t})=\exp \left\{\frac{-\gamma_{D} D_{0} R_{0}}{\psi_{D}+\psi_{R}}\left(e^{\left(\psi_{D}+\psi_{R}\right) \tilde{t}}-1\right)\right\} \text {. }
$$

Therefore,

$$
\operatorname{var}\left[\ln F_{\tilde{t}}\right] \approx \frac{1}{\mathrm{~W}}\left(\exp \left\{\frac{\gamma_{D} D_{0} R_{0}}{\psi_{D}+\psi_{R}}\left(e^{\left(\psi_{D}+\psi_{R}\right) \tilde{t}}-1\right)\right\}-1\right),
$$
estimate,

If we define

$$
\operatorname{var}\left(\Gamma_{\mathrm{LDM}}\right) \approx \frac{1}{\mathrm{~W}}\left(\frac{\psi_{D}+\psi_{R}}{D_{0} R_{0}\left(e^{\left(\psi_{D}+\psi_{R}\right) \tilde{t}}-1\right)}\right)^{2}\left(\exp \left\{\frac{\gamma_{D} D_{0} R_{0}}{\psi_{D}+\psi_{R}}\left(e^{\left(\psi_{D}+\psi_{R}\right) \tilde{t}}-1\right)\right\}-1\right)
$$

$$
\xi_{\tilde{t}}=\frac{\psi_{D}+\psi_{R}}{D_{0} R_{0}\left(e^{\left(\psi_{D}+\psi_{R}\right) \tilde{t}}-1\right)}
$$

then we have (lower variance) for much of the time range. However, if the time gets too high ( $\tilde{t} \approx 5$ for 

variance remains very low). In a case like this, the LDM is predicted to be more precise when the time of the assay is sufficiently low.

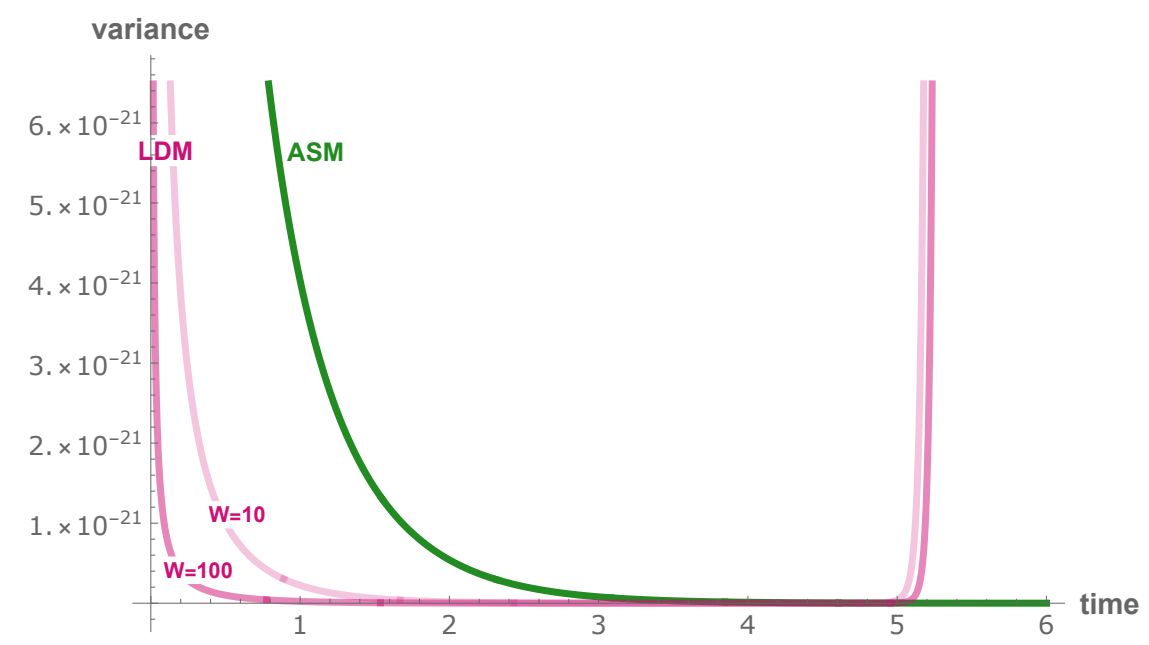

SI Figure 3 : The variance of the ASM (green) and LDM (pink) estimates. Different numbers of populations (W) are used for the LDM estimates, as indicated. The parameters used here are $\gamma_{D}=10^{-12}, D_{0}=R_{0}=10^{4}, \psi_{D}=1$, and $\psi_{R}=\psi_{T}=1.5$.

To show mathematically that the LDM estimate is more precise for short times, we will approximate the expressions for the variance when $\tilde{t}$ is very small. This enables us to use a first-order Maclaurin approximation $e^{c \tilde{t}} \approx 1+c \tilde{t}$. This allows the following approximation of the variance for the ASM estimate: $\operatorname{var}\left(\Gamma_{\mathrm{ASM}}\right)$

We can then simplify this expression:

$$
\begin{gathered}
\operatorname{var}\left(\Gamma_{\mathrm{ASM}}\right) \approx \frac{\gamma_{D}\left(\psi_{D}+\psi_{R}-\psi_{T}\right)}{D_{0} R_{0}}\left\{\frac{\left(\psi_{D}+\psi_{R}\right)\left(\psi_{D}+\psi_{R}\right) \tilde{t}+\left(\psi_{D}+\psi_{R}-2 \psi_{T}\right) \psi_{T} \tilde{t}-2\left(\psi_{D}+\psi_{R}-\psi_{T}\right) 2 \psi_{T} \tilde{t}}{\left(\psi_{D}+\psi_{R}-2 \psi_{T}\right)\left(\psi_{D}+\psi_{R}-\psi_{T}\right)^{2} \tilde{t}^{2}}\right\}, \\
\operatorname{var}\left(\Gamma_{\mathrm{ASM}}\right) \approx \frac{\gamma_{D}}{D_{0} R_{0} \tilde{t}}\left\{\frac{\left(\psi_{D}+\psi_{R}\right)\left(\psi_{D}+\psi_{R}\right)+\left(\psi_{D}+\psi_{R}-2 \psi_{T}\right) \psi_{T}-2\left(\psi_{D}+\psi_{R}-\psi_{T}\right) 2 \psi_{T}}{\left(\psi_{D}+\psi_{R}-2 \psi_{T}\right)\left(\psi_{D}+\psi_{R}-\psi_{T}\right)}\right\},
\end{gathered}
$$

$\operatorname{var}\left(\Gamma_{\mathrm{ASM}}\right)$

$$
\approx \frac{\gamma_{D}}{D_{0} R_{0} \tilde{t}}\left\{\frac{\left(\psi_{D}+\psi_{R}-2 \psi_{T}\right)\left(\psi_{D}+\psi_{R}\right)+2 \psi_{T}\left(\psi_{D}+\psi_{R}\right)+\left(\psi_{D}+\psi_{R}-2 \psi_{T}\right) \psi_{T}-2\left(\psi_{D}+\psi_{R}-\psi_{T}\right) 2 \psi_{T}}{\left(\psi_{D}+\psi_{R}-2 \psi_{T}\right)\left(\psi_{D}+\psi_{R}-\psi_{T}\right)}\right\},
$$

$\operatorname{var}\left(\Gamma_{\mathrm{ASM}}\right)$

$$
\approx \frac{\gamma_{D}}{D_{0} R_{0} \tilde{t}}\left\{\frac{\left(\psi_{D}+\psi_{R}-2 \psi_{T}\right)\left(\psi_{D}+\psi_{R}\right)+2 \psi_{T} \psi_{D}+2 \psi_{T} \psi_{R}+\psi_{T} \psi_{D}+\psi_{T} \psi_{R}-2 \psi_{T}^{2}-4 \psi_{T} \psi_{D}-4 \psi_{T} \psi_{R}+4 \psi_{T}^{2}}{\left(\psi_{D}+\psi_{R}-2 \psi_{T}\right)\left(\psi_{D}+\psi_{R}-\psi_{T}\right)}\right\},
$$

$$
\operatorname{var}\left(\Gamma_{\mathrm{ASM}}\right) \approx \frac{\gamma_{D}}{D_{0} R_{0} \tilde{t}}\left\{\frac{\left(\psi_{D}+\psi_{R}-2 \psi_{T}\right)\left(\psi_{D}+\psi_{R}\right)-\psi_{T} \psi_{D}-\psi_{T} \psi_{R}+2 \psi_{T}^{2}}{\left(\psi_{D}+\psi_{R}-2 \psi_{T}\right)\left(\psi_{D}+\psi_{R}-\psi_{T}\right)}\right\},
$$




$$
\begin{gathered}
\operatorname{var}\left(\Gamma_{\mathrm{ASM}}\right) \approx \frac{\gamma_{D}}{D_{0} R_{0} \tilde{t}}\left\{\frac{\left(\psi_{D}+\psi_{R}-2 \psi_{T}\right)\left(\psi_{D}+\psi_{R}\right)-\left(\psi_{D}+\psi_{R}-2 \psi_{T}\right) \psi_{T}}{\left(\psi_{D}+\psi_{R}-2 \psi_{T}\right)\left(\psi_{D}+\psi_{R}-\psi_{T}\right)}\right\}, \\
\operatorname{var}\left(\Gamma_{\mathrm{ASM}}\right) \approx \frac{\gamma_{D}}{D_{0} R_{0} \tilde{t}}\left\{\frac{\left(\psi_{D}+\psi_{R}-2 \psi_{T}\right)\left(\psi_{D}+\psi_{R}-\psi_{T}\right)}{\left(\psi_{D}+\psi_{R}-2 \psi_{T}\right)\left(\psi_{D}+\psi_{R}-\psi_{T}\right)}\right\}, \\
\operatorname{var}\left(\Gamma_{\mathrm{ASM}}\right) \approx \frac{\gamma_{D}}{D_{0} R_{0} \tilde{t}} .
\end{gathered}
$$

We now turn to the variance for the LDM (which we note is already an approximation). For very small $\tilde{t}$

$$
\begin{gathered}
\xi_{\tilde{t}} \approx \frac{\psi_{D}+\psi_{R}}{D_{0} R_{0}\left(1+\left(\psi_{D}+\psi_{R}\right) \tilde{t}-1\right)}, \\
\xi_{\tilde{t}} \approx \frac{1}{D_{0} R_{0} \tilde{t}} .
\end{gathered}
$$

Thus, we have

$$
\operatorname{var}\left(\Gamma_{\mathrm{LDM}}\right) \approx \frac{\left(\frac{1}{D_{0} R_{0} \tilde{t}}\right)^{2}}{\mathrm{~W}}\left(e^{\left(\gamma_{D} D_{0} R_{0} \tilde{t}\right)}-1\right) .
$$

Using the Maclaurin approximation again yields

$$
\begin{gathered}
\operatorname{var}\left(\Gamma_{\mathrm{LDM}}\right) \approx \frac{\left(\frac{1}{D_{0} R_{0} \tilde{t}}\right)^{2}}{\mathrm{~W}}\left(1+\gamma_{D} D_{0} R_{0} \tilde{t}-1\right), \\
\operatorname{var}\left(\Gamma_{\mathrm{LDM}}\right) \approx \frac{\gamma_{D}}{\mathrm{~W} D_{0} R_{0} \tilde{t}} .
\end{gathered}
$$

Our LDM assay requires $\mathrm{W}>1$. Therefore when $\tilde{t}$ is very small

$$
\operatorname{var}\left(\Gamma_{\mathrm{LDM}}\right)<\operatorname{var}\left(\Gamma_{\mathrm{ASM}}\right) \text {. }
$$

Again, we note the caveat that our estimate for the variance of the LDM estimate was already an approximation.

\section{References}

1. Redondo-Salvo, S. et al. Pathways for horizontal gene transfer in bacteria revealed by a global map of their plasmids. Nat. Commun. 11, 3602 (2020).

2. Loftie-Eaton, W. et al. Compensatory mutations improve general permissiveness to antibiotic resistance plasmids. Nat. Ecol. Evol. 1, 1354-1363 (2017).

3. Norman, A., Hansen, L. H. \& Sørensen, S. J. Conjugative plasmids: vessels of the communal gene pool. Philos. Trans. R. Soc. B Biol. Sci. 364, 2275-2289 (2009). 
1917 4. Jordt, H. et al. Coevolution of host-plasmid pairs facilitates the emergence of novel multidrug resistance. Nat. Ecol. Evol. 1-7 (2020) doi:10.1038/s41559-020-1170-1.

5. Dahlberg, C. \& Chao, L. Amelioration of the Cost of Conjugative Plasmid Carriage in Eschericha coli K12. Genetics 165, 1641-1649 (2003).

7. Lopatkin, A. J. et al. Persistence and reversal of plasmid-mediated antibiotic resistance. Nat. Commun. 8, 1-10 (2017).

6. Stewart, F. M. \& Levin, B. R. The Population Biology of Bacterial Plasmids: A Priori Conditions for the Existence of Conjugationally Transmitted Factors. Genetics 87, 209-228 (1977).

\author{
8. Fox, R. E., Zhong, X., Krone, S. M. \& Top, E. M. Spatial structure and nutrients
} promote invasion of IncP-1 plasmids in bacterial populations. ISME J. 2, 1024-1039 (2008).

9. Alderliesten, J. B. et al. Effect of donor-recipient relatedness on the plasmid conjugation frequency: a meta-analysis. BMC Microbiol. 20, 135 (2020).

10. Zhong, X., Droesch, J., Fox, R., Top, E. M. \& Krone, S. M. On the meaning and estimation of plasmid transfer rates for surface-associated and well-mixed bacterial populations. J. Theor. Biol. 294, 144-152 (2012).

11. Huisman, J. S. et al. Estimating the rate of plasmid transfer in liquid mating cultures. bioRxiv 2020.03.09.980862 (2020) doi:10.1101/2020.03.09.980862.

12. Simonsen, L. The Existence Conditions for Bacterial Plasmids: Theory and Reality. Microb. Ecol. 22, 187-205 (1991).

13. Levin, B. R., Stewart, F. M. \& Rice, V. A. The kinetics of conjugative plasmid transmission: Fit of a simple mass action model. Plasmid 2, 247-260 (1979). 
14. Zhong, X., Droesch, J., Fox, R., Top, E. M. \& Krone, S. M. On the meaning and estimation of plasmid transfer rates for surface-associated and well-mixed bacterial populations. J. Theor. Biol. 294, 144-152 (2012).

15. Bethke, J. H. et al. Environmental and genetic determinants of plasmid mobility in pathogenic Escherichia coli. Sci. Adv. 6, eaax3173 (2020).

16. Simonsen, L., Gordon, D. M., Stewart, F. M. \& Levin, B. R. Estimating the rate of plasmid transfer: an end-point method. Microbiology, 136, 2319-2325 (1990).

17. Lopatkin, A. J. et al. Antibiotics as a selective driver for conjugation dynamics. Nat.

18. San Millan, A. Evolution of Plasmid-Mediated Antibiotic Resistance in the Clinical Context. Trends Microbiol. 26, 978-985 (2018).

19. Li, L. et al. Plasmids persist in a microbial community by providing fitness benefit to multiple phylotypes. ISME J. 1-12 (2020) doi:10.1038/s41396-020-0596-4.

20. Luria, S. E. \& Delbrück, M. Mutations of Bacteria from Virus Sensitivity to Virus Resistance. Genetics 28, 491-511 (1943).

21. Johnsen, A. R. \& Kroer, N. Effects of stress and other environmental factors on horizontal plasmid transfer assessed by direct quantification of discrete transfer events. FEMS Microbiol. Ecol. 59, 718-728 (2007).

22. Loftie-Eaton, W. et al. Evolutionary Paths That Expand Plasmid Host-Range: Implications for Spread of Antibiotic Resistance. Mol. Biol. Evol. 33, 885-897 (2016). 23. Yano, H. et al. Evolved plasmid-host interactions reduce plasmid interference cost. Mol. Microbiol. 101, 743-756 (2016). 
24. Valle, A. A. et al. The distribution of plasmid fitness effects explains plasmid persistence in bacterial communities. bioRxiv 2020.08.01.230672 (2020) doi:10.1101/2020.08.01.230672.

25. Abboud, R. et al. Low-Temperature Growth of Shewanella oneidensis MR-1. Appl. Environ. Microbiol. 71, 811-816 (2005).

26. Gibson, B., Wilson, D. J., Feil, E. \& Eyre-Walker, A. The distribution of bacterial

30. Turner, P. E. et al. Antibiotic resistance correlates with transmission in plasmid evolution. Evolution 68, 3368-3380 (2014).

31. Turner, P. E., Cooper, V. S. \& Lenski, R. E. Tradeoff Between Horizontal and Vertical Modes of Transmission in Bacterial Plasmids. Evolution 52, 315-329 (1998).

32. Kottara, A., Hall, J. P. J., Harrison, E. \& Brockhurst, M. A. Multi-host environments select for host-generalist conjugative plasmids. BMC Evol. Biol. 16, 70 (2016). natural populations. Microbiology, 138, 17-21 (1992). 
34. Philipsen, K. R., Christiansen, L. E., Hasman, H. \& Madsen, H. Modelling conjugation with stochastic differential equations. J. Theor. Biol. 263, 134-142 (2010).

35. Dimitriu, T., Matthews, A. \& Buckling, A. Increased copy number couples the evolution of plasmid horizontal transmission and antibiotic resistance. http://biorxiv.org/lookup/doi/10.1101/2020.08.12.248336 (2020) doi:10.1101/2020.08.12.248336.

36. Szpirer, C., Top, E., Couturier, M. \& Mergeay, M. Retrotransfer or gene capture: a 1993 feature of conjugative plasmids, with ecological and evolutionary significance. Microbiology, 145, 3321-3329 (1999).

37. Kneis, D., Hiltunen, T. \& Heß, S. A high-throughput approach to the culture-based estimation of plasmid transfer rates. Plasmid 101, 28-34 (2019). population. J. Stat. Mech. Theory Exp. 2015, P01011 (2015). 